MATHEMATICS OF COMPUTATION

Volume 79, Number 272, October 2010, Pages 1957-1999

S 0025-5718(10)02341-0

Article electronically published on April 21, 2010

\title{
CONVERGENT FINITE ELEMENT DISCRETIZATION OF THE MULTI-FLUID NONSTATIONARY INCOMPRESSIBLE MAGNETOHYDRODYNAMICS EQUATIONS
}

\author{
ĽUBOMÍR BAŇAS AND ANDREAS PROHL
}

\begin{abstract}
We propose a convergent implicit stabilized finite element discretization of the nonstationary incompressible magnetohydrodynamics equations with variable density, viscosity, and electric conductivity. The discretization satisfies a discrete energy law, and a discrete maximum principle for the positive density, and iterates converge to weak solutions of the limiting problem for vanishing discretization parameters. A simple fixed point scheme, together with an appropriate stopping criterion is proposed, which decouples the computation of density, velocity, and magnetic field, and inherits the above properties, provided a mild mesh constraint holds. Computational studies are provided.
\end{abstract}

\section{INTRODUCTION}

We consider the density-dependent magneto-hydrodynamics equations, which couples the incompressible Navier-Stokes equation with variable density and viscosity with Maxwell's equation to describe a viscous, incompressible, and electrically conducting multi-fluid in a domain $\Omega \subset \mathbb{R}^{d}(d=2,3)$ :

$$
\begin{aligned}
(\rho \mathbf{u})_{t}+\operatorname{div}(\rho \mathbf{u} \otimes \mathbf{u})-\operatorname{div}(\eta(\rho) \boldsymbol{D}(\mathbf{u})) & =-\nabla p+\rho \mathbf{g}+\frac{1}{\bar{\mu}} \operatorname{curl} \mathbf{b} \times \mathbf{b}, \\
\operatorname{div} \mathbf{u} & =0, \\
\rho_{t}+\operatorname{div}(\rho \mathbf{u}) & =0, \\
\mathbf{b}_{t}+\frac{1}{\bar{\mu}} \operatorname{curl}\left(\frac{1}{\xi(\rho)} \operatorname{curl} \mathbf{b}\right) & =\operatorname{curl}(\mathbf{u} \times \mathbf{b}), \\
\operatorname{div} \mathbf{b} & =0,
\end{aligned}
$$

together with the following boundary and initial conditions,

$$
\begin{aligned}
& \mathbf{u}=\mathbf{c u r l} \mathbf{b} \times \mathbf{n}=\mathbf{0}, \quad \mathbf{b} \cdot \mathbf{n}=0 \quad \text { on } \partial \Omega_{T}:=(0, T) \times \partial \Omega, \\
& \mathbf{u}(0, \cdot)=\mathbf{u}_{0}, \quad \mathbf{b}(0, \cdot)=\mathbf{b}_{0} \quad \text { in } \Omega, \quad \rho(0, \mathbf{x})=\rho_{0} \\
& \quad=\left\{\begin{array}{ll}
\bar{\rho}_{1}>0, & \text { constant on } \Omega_{1}, \\
\bar{\rho}_{2}>0, & \text { constant on } \Omega_{2},
\end{array} \text { with } \bar{\Omega}_{1} \cup \bar{\Omega}_{2}=\bar{\Omega}, \quad \operatorname{meas}\left(\Omega_{i}\right)>0 .\right.
\end{aligned}
$$

Received by the editor December 4, 2008 and, in revised form, August 7, 2009.

2010 Mathematics Subject Classification. Primary 65M60, 65M12, 76W05; Secondary 65M55, $65 \mathrm{M} 50$.

(C)2010 American Mathematical Society

Reverts to public domain 28 years from publication 
Here, $\mathbf{u}: \Omega_{T} \rightarrow \mathbb{R}^{d}$ denotes the velocity field, $\boldsymbol{D}(\mathbf{u})=\frac{1}{2}\left(\nabla \mathbf{u}+[\nabla \mathbf{u}]^{\top}\right)$ the symmetrized velocity gradient, $\rho: \Omega_{T} \rightarrow \mathbb{R}^{+}$the density, and $p: \Omega_{T} \rightarrow \mathbb{R}$ the hydrodynamic pressure of vanishing mean value, i.e., $\int_{\Omega} p(\cdot, \mathbf{x}) \mathrm{d} \mathbf{x}=0$. Moreover, $\mathbf{b}: \Omega_{T} \rightarrow \mathbb{R}^{d}$ is the magnetic induction, and $\mathbf{g}: \Omega_{T} \rightarrow \mathbb{R}^{d}$ represents a given body force per unit mass. We allow for a discontinuous density dependent viscosity $\eta(\rho)$, and an electric conductivity $\xi(\rho)$, where $\eta, \xi$ are continuous functions such that

$$
0<\bar{\eta}_{-} \leq \eta \leq \bar{\eta}_{+}, \quad 0<\bar{\xi}_{-} \leq \xi \leq \bar{\xi}_{+} .
$$

The initial datum $\rho_{0}: \Omega \rightarrow \mathbb{R}$ is piecewise constant, positive; as is pointed out in [11. Section 4.2], choosing $\rho_{0} \geq 0$ is mathematically feasible (also in this work), but makes the physical relevancy of equation (1.1) 4 questionable in zones of vacuum. Hence, the case of fluids enclosed in a free surface and surrounded by a vacuum is excluded, while the evolution of a free interface separating two fluids filling $\Omega$ is modelled by (1.1)-(1.2). This problem is mostly relevant in the case $d=3$, but the two-dimensional analogue is interesting as well; see e.g. [17, Remark 2.1] for a proper definition of involved curl-operators in the case $d=2$.

Crucial properties of solutions of (1.1) are nonnegativity, a maximum principle for $\rho$, which amounts to

$$
\bar{\rho}_{1} \leq \rho \leq \bar{\rho}_{2} \quad \text { a.e. in } \Omega_{T},
$$

as well as the energy law $(0<t<T)$ :

$$
\frac{d}{d t} \int_{\Omega}\left[\frac{\rho|\mathbf{u}|^{2}}{2}+\frac{|\mathbf{b}|^{2}}{2 \bar{\mu}}\right] \mathrm{d} \mathbf{x}+\int_{\Omega}\left[\eta(\rho)|\boldsymbol{D}(\mathbf{u})|^{2}+\frac{1}{\bar{\mu}^{2} \xi(\rho)}|\mathbf{c u r l} \mathbf{b}|^{2}\right] \mathrm{d} \mathbf{x}=\int_{\Omega} \rho \mathbf{g} \cdot \mathbf{u} \mathrm{d} \mathbf{x} .
$$

We refer to [11, Chapter 4 and 5] for details regarding the model, and existing analytical and numerical studies of these equations. A typical application of (1.1)(1.2) is to describe the production of aluminium in electrolytic cells. A schematic description of this experiment uses an electric current which runs downwards through two horizontal layers of incompressible nonmiscible conducting fluids, and causes a motion of the interface, depending on the magnetohydrodynamic coupling; see [11, Chapter 6], and Section [5.

Weak solutions to (1.1)-1.2) are constructed in 11, Chapter 4] for $\Omega \subset \mathbb{R}^{3}$ a simply connected, regular domain. It is well known that $\mathbf{H}_{0}(\operatorname{div} ; \Omega) \cap \mathbf{H}(\mathbf{c u r l} ; \Omega)$ is continuously embedded into $\mathbf{W}^{1,2}(\Omega)$ if $\Omega \subset \mathbb{R}^{3}$ is a convex polyhedron, or has a boundary which is $C^{1,1}$; cf. [15, Section I.3.5] or [1, Section 2.3]. In this work, we propose fully practical finite element based schemes, whose solutions converge to weak solutions for vanishing discretization parameters; in particular, this result holds for $\Omega \subset \mathbb{R}^{3}$ a general polyhedral domain. Key tools to accomplish this result are:

(i) a discrete energy and maximum principle for approximate densities, which requires stabilization terms in the discrete setting (Lemma 3.1),

(ii) an adapted discrete version (Lemma 3.2) of the compactness result by R. DiPerna and P.L. Lions [9] for approximate finite element solutions of (1.1) 3 ; see also Remark 3.1.

(iii) a compactness result of J.L. Lions to control temporal changes of iterates (see Lemma 3.3), and

(iv) results from corresponding studies for a one-fluid magnetohydrodynamical fluid flow in [33. 
The paper is organized as follows. Section 2 gathers necessary background material. Scheme A is presented in Section 3, where solutions satisfy a discrete energy law, and corresponding positive densities a discrete maximum principle; a proper balancing of involved stabilization terms to construct weak solutions of (1.1)-(1.2) for vanishing discretization parameters is needed. In order to solve the nonlinear algebraic system, the decoupling iterative Algorithm A is proposed in Section 4. under a mild mesh constraint, the scheme terminates, and iterates converge towards solutions of Scheme A provided a suitable stopping parameter approaches zero. In Section 5 we discuss a practical implementation of the scheme and perform numerical experiments similar to corresponding studies in [11, Chapter 6] for aluminium electrolysis.

\section{Preliminaries}

Below, always let $\Omega \subset \mathbb{R}^{d}$, for $d=2,3$ be a simply connected, bounded smooth domain. We use boldface letters for vector-valued functions.

2.1. Functional spaces and notion of weak solution for MHD equations. Let $\mathbf{H}(\operatorname{div} ; \Omega)$ be the set of vector fields $\boldsymbol{\xi} \in \mathbf{L}^{2}(\Omega)$, such that $\operatorname{div} \boldsymbol{\xi} \in L^{2}(\Omega)$; the space is endowed with the norm $\|\boldsymbol{\xi}\|_{\mathbf{H}(\mathrm{div})}:=\left(\|\boldsymbol{\xi}\|_{\mathbf{L}^{2}}^{2}+\|\operatorname{div} \boldsymbol{\xi}\|_{L^{2}}^{2}\right)^{1 / 2}$. We introduce the following subspaces $\mathbf{H}_{0}(\operatorname{div} ; \Omega):=\{\boldsymbol{\xi} \in \mathbf{H}(\operatorname{div} ; \Omega): \boldsymbol{\xi} \cdot \mathbf{n}=0$ on $\partial \Omega\}$, and

$$
\begin{aligned}
\mathcal{J} & :=\left\{\boldsymbol{\xi} \in \mathbf{C}_{0}^{\infty}(\Omega): \operatorname{div} \boldsymbol{\xi}=0 \text { in } \Omega\right\}, \\
\mathbf{H} & :=\left\{\boldsymbol{\xi} \in \mathbf{L}^{2}(\Omega): \operatorname{div} \boldsymbol{\xi}=0 \text { weakly in } \Omega, \text { and } \boldsymbol{\xi} \cdot \mathbf{n}=0 \text { on } \partial \Omega\right\}, \\
\mathbf{J} & :=\left\{\boldsymbol{\xi} \in \mathbf{W}_{0}^{1,2}(\Omega): \operatorname{div} \boldsymbol{\xi}=0 \text { a.e. in } \Omega\right\} .
\end{aligned}
$$

By Helmholtz orthogonal decomposition, a vector field can be written as a unique sum of its solenoidal and irrotational components, i.e., $\mathbf{L}^{2}(\Omega)=\mathbf{H} \oplus \nabla W^{1,2}(\Omega)$. Correspondingly, we introduce the space $\mathbf{H}(\operatorname{curl} ; \Omega)$, which is endowed with the norm $\|\boldsymbol{\psi}\|_{\mathbf{H}(\mathbf{c u r l})}:=\left(\|\boldsymbol{\psi}\|_{\mathbf{L}^{2}}^{2}+\|\operatorname{curl} \boldsymbol{\psi}\|_{\mathbf{L}^{2}}^{2}\right)^{1 / 2}$, and subspaces $\mathbf{H}_{0}(\operatorname{curl} ; \Omega):=\{\boldsymbol{\psi} \in$ $\mathbf{H}(\mathbf{c u r l} ; \Omega): \boldsymbol{\psi} \times \mathbf{n}=0$ on $\partial \Omega\}$, as well as

$$
\begin{aligned}
\mathcal{W} & :=\left\{\boldsymbol{\psi} \in \mathbf{C}^{\infty}(\bar{\Omega}): \operatorname{div} \boldsymbol{\psi}=0 \text { in } \Omega, \boldsymbol{\psi} \cdot \mathbf{n}=0 \text { on } \partial \Omega\right\}, \\
\mathcal{H} & :=\mathbf{H}(\operatorname{curl} ; \Omega) \cap \mathbf{H}_{0}(\operatorname{div} ; \Omega), \\
\mathbf{X} & :=\{\boldsymbol{\psi} \in \mathcal{H}: \operatorname{div} \boldsymbol{\psi}=0 \text { a.e. in } \Omega\} .
\end{aligned}
$$

The space $\mathcal{D}(\bar{\Omega})$ of restrictions to $\bar{\Omega}$ of smooth functions $\mathcal{D}\left(\mathbb{R}^{3}\right)$ is dense, both in $\mathbf{H}(\operatorname{div} ; \Omega)$ and $\mathbf{H}(\mathbf{c u r l} ; \Omega)$; cf. [1, Proposition 2.3]. For all $\boldsymbol{\psi} \in \mathbf{X}$, we have Poincaré's inequality $\|\boldsymbol{\psi}\|_{\mathbf{L}^{2}} \leq C\|\operatorname{curl} \boldsymbol{\psi}\|_{\mathbf{L}^{2}}$ for all $\boldsymbol{\psi} \in \mathbf{X}$, which implies that $\|\cdot\|_{\mathbf{H}(\mathbf{c u r l})}$ and $\|\operatorname{curl}(\cdot)\|_{\mathbf{L}^{2}}$ are equivalent norms on $\mathbf{X}$. The space $\mathbf{H}_{0}(\mathbf{c u r l} ; \Omega) \cap$ $\mathbf{H}_{0}(\operatorname{div} ; \Omega)$ coincides with $\mathbf{W}_{0}^{1,2}(\Omega)$; in contrast, explicit counterexamples illustrate that $\mathbf{H}_{0}(\operatorname{div} ; \Omega) \cap \mathbf{H}(\mathbf{c u r l} ; \Omega)$ is continuously embedded into $\mathbf{W}^{1,2}(\Omega)$ only if $\Omega \subset \mathbb{R}^{3}$ is either a convex polyhedron or has a boundary which is $C^{1,1}$; cf. [15, Section I.3.5] or [1, Section 2.3]. The embeddings of $\mathbf{H}(\mathbf{c u r l} ; \Omega)$ and $\mathbf{H}(\operatorname{div} ; \Omega)$ into $\mathbf{L}^{2}(\Omega)$ are not compact; however, C. Weber verified compactness of $\mathbf{H}_{0}(\mathbf{c u r l} ; \Omega)$ and $\mathbf{H}_{0}(\operatorname{div} ; \Omega)$ into $\mathbf{L}^{2}(\Omega)$ for general Lipschitz polyhedral domains; the following compactness results may be found in [1, Proposition 3.7] and [36, Proposition 2.3]. 
Lemma 2.1. Let $\Omega \subset \mathbb{R}^{3}$ be a Lipschitz polyhedron.

(i) There exists an exponent $s \equiv s(\Omega)>\frac{1}{2}$, such that $\mathcal{H} \hookrightarrow \mathbf{W}^{s, 2}(\Omega)$ is continuous.

(ii) There exists a parameter $\delta_{1} \equiv \delta_{1}(\Omega)>0$ such that the embedding $\mathcal{H} \hookrightarrow$ $\mathbf{L}^{3+\delta_{1}}(\Omega)$ is compact.

We need the Aubin-Lions' compactness result for Bochner spaces; cf. 11, Lemma $2.8]$.

Lemma 2.2. Let $B$ be a Banach space, and $B_{0}$ and $B_{1}$ two reflexive Banach spaces. Assume $B_{0} \Subset B \subset B_{1}$. Fix $T<\infty$, and $1<p_{0}, p_{1}<\infty$. Then

$$
\left\{v \in L^{p_{0}}\left(0, T ; B_{0}\right): \partial_{t} v \in L^{p_{1}}\left(0, T ; B_{1}\right)\right\} \Subset L^{p_{0}}(0, T ; B) .
$$

We recall a crucial tool to construct unique distributional solutions $\rho: \Omega_{T} \rightarrow \mathbb{R}$ of the convection equation

$$
\rho_{t}+\operatorname{div}(\mathbf{u} \rho)=f \quad \text { in } \Omega_{T}, \quad \rho(0, \cdot)=\rho_{0} \in L^{\infty}(\Omega),
$$

for given $\mathbf{u} \in L^{1}\left(0, T ; \mathbf{W}_{0}^{1,2}(\Omega)\right)$, and $f \in L^{1}\left(0, T ; L^{2}(\Omega)\right)$, which has been derived by J. DiPerna and P.L. Lions [9], together with the following stability result (see e.g. [38, Theorem 1.1]).

Lemma 2.3. Let $\left\{\rho_{k}\right\}_{k=0}^{\infty} \subset L^{\infty}\left(0, T ; L^{2}(\Omega)\right)$ be distributional solutions of

$$
\left(\rho_{k}\right)_{t}+\mathbf{u}_{k} \cdot \nabla \rho_{k}+\left[\operatorname{div} \mathbf{u}_{k}\right] \rho_{k}=f_{k} \quad \text { in } \Omega_{T}, \quad \rho_{k}(0, \cdot)=\left(\rho_{k}\right)_{0} \quad \text { in } \Omega .
$$

Assume that

(i) $\left\{\mathbf{u}_{k}\right\}_{k} \subset L^{1}\left(0, T ; \mathbf{W}_{0}^{1,2}(\Omega)\right)$, that $\mathbf{u}_{k} \rightarrow \mathbf{u}$ in $L^{1}\left(0, T ; \mathbf{L}^{2}(\Omega)\right)$, and $\operatorname{div} \mathbf{u}_{k} \rightarrow$ $\operatorname{div} \mathbf{u}$ in $L^{1}\left(0, T ; L^{\infty}(\Omega)\right)$, with $\mathbf{u} \in L^{1}\left(0, T ; \mathbf{W}_{0}^{1,2}(\Omega)\right)$, and

(ii) $f_{k} \rightarrow f$ in $L^{1}\left(0, T ; L^{2}(\Omega)\right)$, and $\left(\rho_{k}\right)_{0} \rightarrow \rho_{0}$ in $L^{2}(\Omega)$.

Then $\rho_{k} \rightarrow \rho$ in $L^{2}\left(0, T ; L^{2}(\Omega)\right)$, where $\rho: \Omega_{T} \rightarrow \mathbb{R}$ is the unique solution of (2.1). Moreover, this solution has the following properties:

(1) $\rho \in C\left([0, T] ; L^{2}(\Omega)\right)$.

(2) If $\beta \in C^{1}(\mathbb{R})$ satisfies $\beta^{\prime}(t) \leq C\left(1+|t|^{r}\right)$, for $C>0$ and $r \leq 1$, then

$$
\frac{d}{d t} \int_{\Omega} \beta(\rho) \mathrm{d} \mathbf{x}+\int_{\Omega}\left[[\operatorname{div} \mathbf{u}]\left(\rho \beta^{\prime}(\rho)-\beta(\rho)\right)\right] \mathrm{d} \mathbf{x}=\int_{\Omega} f \beta^{\prime}(\rho) \mathrm{d} \mathbf{x} .
$$

This compactness result is a crucial tool to establish existence of solutions for the incompressible Navier-Stokes equations with variable density and viscosity [28]. Another compactness result will be useful below to validate strong convergence of both, velocity and magnetic field iterates in $L^{2}\left(0, T ; \mathbf{L}^{2}\right)$; cf. e.g. [27, Ch. I, Sec. 5] for a proof of

Lemma 2.4. Let $B_{0} \subset B \subset B_{1}$ be Banach spaces, $B_{0}$ and $B_{1}$ reflexive, with compact embedding of $B_{0}$ in $B$. Let $p \in(1, \infty)$ and $\alpha \in(0,1)$ be given. Let $X$ be the space $X=L^{p}\left(0, T ; B_{0}\right) \cap W^{\alpha, p}\left(0, T ; B_{1}\right)$ endowed with the natural norm. Then the embedding of $X$ in $L^{p}(0, T ; B)$ is compact. 
2.2. Weak solution of (1.1)-(1.2). The definition of weak solutions of (1.1)-(1.2) below generalizes the one in [11, Definition 4.3] to allow for arbitrary polyhedral domains $\Omega \subset \mathbb{R}^{3}$; solvability will be shown in Section 3 by a practical implicit finite element based discretization.

Definition 2.1. Fix $T>0$. Suppose $\mathbf{f} \in L^{2}\left(0, T ; \mathbf{L}^{2}\right)$, that $\mathbf{u}_{0}, \mathbf{b}_{0} \in \mathbf{H}$, and (1.3) are valid. We call the triple $(\mathbf{u}, \mathbf{b}, \rho): \Omega_{T} \rightarrow \mathbb{R}^{d}$ a weak solution to (1.1)-1.2) if

(i) $\mathbf{u} \in L^{\infty}(0, T ; \mathbf{H}) \cap L^{2}(0, T ; \mathbf{J}), \mathbf{b} \in L^{\infty}(0, T ; \mathbf{H}) \cap L^{2}(0, T ; \mathbf{X})$, and $\rho \in$ $L^{\infty}\left(\Omega_{T}\right) \cap C\left([0, T] ; L^{p}(\Omega)\right)$, for all $p \geq 1$.

(ii) $(\mathbf{u}, \mathbf{b})$ is weakly continuous for $t>0$, with values in $[\mathbf{H}]^{2}$,

(iii) for all $(\boldsymbol{\zeta}, \boldsymbol{\eta}) \in C_{0}^{\infty}([0, T) ; \mathcal{J}) \times C_{0}^{\infty}([0, T) ; \mathcal{W})$, and $\chi \in C_{0}^{\infty}\left([0, T) ; C_{0}^{\infty}(\Omega)\right)$ it holds that

$$
\begin{aligned}
& \int_{0}^{T}[\left.-\left(\rho \mathbf{u}, \boldsymbol{\zeta}_{t}\right)-(\rho \mathbf{u} \otimes \mathbf{u}, \nabla \boldsymbol{\zeta})+(\eta(\rho) \boldsymbol{D}(\mathbf{u}), \boldsymbol{D}(\boldsymbol{\zeta}))\right] \mathrm{d} t \\
&=\int_{0}^{T}\left[(\rho \mathbf{g}, \boldsymbol{\zeta})+\left(\frac{1}{\bar{\mu}} \mathbf{c u r l} \mathbf{b} \times \mathbf{b}, \boldsymbol{\zeta}\right)\right] \mathrm{d} t+\left(\rho_{0} \mathbf{u}_{0}, \boldsymbol{\zeta}(0, \cdot)\right), \\
& \int_{0}^{T}\left[-\left(\rho, \chi_{t}\right)-(\rho \mathbf{u}, \nabla \chi)\right] \mathrm{d} s=\left(\rho_{0}, \chi(0, \cdot)\right) \\
& \int_{0}^{T}\left[-\left(\mathbf{b}, \boldsymbol{\eta}_{t}\right)+\frac{1}{\bar{\mu}}\left(\frac{1}{\xi(\rho)} \operatorname{curl} \mathbf{b}, \operatorname{curl} \boldsymbol{\eta}\right)\right] \mathrm{d} s \\
&=\int_{0}^{T}(\operatorname{curl}(\mathbf{u} \times \mathbf{b}), \boldsymbol{\eta}) \mathrm{d} s+\left(\mathbf{b}_{0}, \boldsymbol{\eta}(0, \cdot)\right) .
\end{aligned}
$$

(iv) for almost every $t \in[0, T]$,

$$
\frac{d}{d t} \int_{\Omega}\left[\frac{\rho|\mathbf{u}|^{2}}{2}+\frac{|\mathbf{b}|^{2}}{2 \bar{\mu}}\right] \mathrm{d} \mathbf{x}+\int_{\Omega}\left[\eta(\rho)|\boldsymbol{D}(\mathbf{u})|^{2}+\frac{1}{\bar{\mu}^{2} \xi(\rho)}|\mathbf{c u r l} \mathbf{b}|^{2}\right] \mathrm{d} \mathbf{x} \leq \int_{\Omega} \rho \mathbf{g} \cdot \mathbf{u} \mathrm{d} \mathbf{x} .
$$

Weak solutions to problem (1.1)-(1.2) are constructed in [11, Theorem 4.4] for domains $\Omega \subset \mathbb{R}^{3}$ which are either convex, or have a boundary in $C^{1,1}$.

2.3. Finite element spaces. Let $\mathcal{T}_{h}$ be a quasi-uniform triangulation of the polygonal, resp., polyhedral domain $\Omega \subset \mathbb{R}^{3}$ into tetrahedra of maximal diameter $h>0$, i.e., $\bar{\Omega}=\bigcup_{K \in \mathcal{T}_{h}} \bar{K}$. Throughout this work, let $C>0$ denote a generic constant which does not depend on time and space discretization parameters $k, h>0$.

Discrete density space. Let $V_{h}:=\left\{\xi \in C(\bar{\Omega}): \xi \in P_{1}(K) \forall K \in \mathcal{T}_{h}\right\}$, where $P_{1}$ is the set of piecewise affine polynomials. We recall the inverse inequality [4, Lemma 4.5.3], which holds for $\xi \in V_{h}$,

$$
\|\xi\|_{W^{\ell, q_{1}}} \leq C h^{m-\ell+d \min \left\{\frac{1}{q_{1}}-\frac{1}{q_{2}}, 0\right\}}\|\xi\|_{W^{m, q_{2}}} \quad \forall 1 \leq q_{1}, q_{2} \leq \infty, \quad 0 \leq m \leq \ell .
$$

Given the set of nodes $\mathcal{E}_{h}=\left\{\mathbf{x}_{\ell}: \ell \in L\right\}$ of the triangulation $\mathcal{T}_{h}$, the nodal interpolation operator $\mathcal{I}_{V_{h}}: C(\bar{\Omega}, \mathbb{R}) \rightarrow V_{h}$ satisfies $\mathcal{I}_{V_{h}} \xi\left(\mathbf{x}_{\ell}\right)=\xi\left(\mathbf{x}_{\ell}\right)$ for all $\ell \in L$. For continuous functions $\phi, \psi \in C(\bar{\Omega}, \mathbb{R})$ we define

$$
(\phi, \psi)_{h}=\int_{\Omega} \mathcal{I}_{V_{h}}(\phi \psi) \mathrm{d} \mathbf{x}=\sum_{\ell \in L} \beta_{\ell} \phi\left(\mathbf{x}_{\ell}\right) \psi\left(\mathbf{x}_{\ell}\right),
$$

for certain weights $\beta_{\ell}>0, \ell \in L$. If for each $\ell \in L$ we denote by $\varphi_{\ell} \in C(\bar{\Omega})$ the nodal basis function which is $\mathcal{T}_{h}$ elementwise affine and satisfies $\varphi_{\ell}\left(\mathbf{x}_{\ell}\right)=1$ 
and $\varphi_{\ell}\left(\mathbf{x}_{m}\right)=0$ for all $m \in L \backslash\{\ell\}$, then we have $\beta_{\ell}=\int_{\Omega} \varphi_{\ell} \mathrm{d} \mathbf{x}$. We define $\|\xi\|_{h}^{2}=(\xi, \xi)_{h}$, and recall (see [6]) that for all $\xi, \chi, \eta \in V_{h}$,

$$
\begin{aligned}
& \|\xi\|^{2} \leq\|\xi\|_{h}^{2} \leq(d+2)\|\xi\|^{2}, \\
& \left|(\chi, \eta)-(\chi, \eta)_{h}\right| \leq C h\|\chi\|\|\eta\|_{H^{1}} .
\end{aligned}
$$

We say that the triangulation $\mathcal{T}_{h}$ is strongly acute if for all $\mathbf{x}_{\ell} \in \mathcal{E}_{h} \backslash \partial \Omega$ and all $\mathbf{x}_{\ell^{\prime}} \in \mathcal{E}_{h} \backslash\left\{\mathbf{x}_{\ell}\right\}$ there exists $C_{\theta_{0}}>0$ such that $\left(\nabla \varphi_{\mathbf{x}_{\ell}}, \nabla \varphi_{\mathbf{x}_{\ell^{\prime}}}\right) \leq-C_{\theta_{0}} h^{d-2}$. If $d=2$ this holds if and only if every sum of two angles opposite to an interior edge is bounded by $\pi-\theta_{0}$, for some $\theta>0$. For $d=3$, a sufficient but not necessary condition is that every angle between two faces of a tetrahedron is bounded by $\frac{\pi}{2}-\theta_{0}$, for some $\theta_{0}>0$; see [24] for further details.

Discrete velocity and pressure spaces. Let $\mathbf{V}_{h}:=\left[\tilde{V}_{h}\right]^{3}$, where $\tilde{V}_{h}$ is another $W_{0}^{1,2}$-conforming finite element space. We define $\mathbf{L}^{2}(\Omega)$-, resp., $\mathbf{W}_{0}^{1,2}(\Omega)$ orthonormal projections $\mathbf{P}_{h}^{0}$, resp., $\mathbf{P}_{h}^{1}$ to $\mathbf{V}_{h}$,

$$
\left(\mathbf{z}-\mathbf{P}_{h}^{0} \mathbf{z}, \boldsymbol{\xi}\right)=0 \quad \forall \boldsymbol{\xi} \in \mathbf{V}_{h}, \quad \text { resp. }, \quad\left(\nabla\left[\mathbf{z}-\mathbf{P}_{h}^{1} \mathbf{z}\right], \nabla \boldsymbol{\xi}\right)=0 \quad \forall \boldsymbol{\xi} \in \mathbf{V}_{h} .
$$

We define the nodal interpolation operator $\mathcal{I}_{\mathbf{V}_{h}}: C\left(\bar{\Omega}, \mathbb{R}^{3}\right) \rightarrow \mathbf{V}_{h}$, such that $\mathcal{I}_{\mathbf{V}_{h}} \boldsymbol{\xi}:=\sum_{\mathbf{z} \in \mathcal{E}_{h}} \boldsymbol{\xi}(\mathbf{z}) \bar{\varphi}_{\mathbf{z}}$, where $\left\{\bar{\varphi}_{\mathbf{z}}: \mathbf{z} \in \mathcal{E}_{h}\right\}$ denotes the nodal basis of $\tilde{V}_{h}$.

For $j \geq 0$, consider $L_{h}:=\left\{\Pi \in L_{0}^{2}(\Omega): \Pi \in P_{j}(K) \forall K \in \mathcal{T}_{h}\right\}$. The spaces $\left(\mathbf{V}_{h}, L_{h}\right)$ are chosen such that the discrete LBB-condition holds, i.e., $\mathbf{V}_{h} \subset$ $\mathbf{W}_{0}^{1,2}\left(\Omega, \mathbb{R}^{3}\right)$ and $L_{h} \subset L_{0}^{2}(\Omega)$ satisfy the discrete inf-sup condition

$$
\sup _{\boldsymbol{\xi} \in \mathbf{V}_{h}} \frac{(\operatorname{div} \boldsymbol{\xi}, \Pi)}{\|\nabla \boldsymbol{\xi}\|_{\mathbf{L}^{2}}} \geq C\|\Pi\|_{L^{2}} \quad \forall \Pi \in L_{h} .
$$

Let

$$
\mathbf{J}_{h}:=\left\{\boldsymbol{\xi} \in \mathbf{V}_{h}:(\operatorname{div} \boldsymbol{\xi}, \Pi)=0 \text { for all } \Pi \in L_{h}\right\} \not \subset \mathbf{J}
$$

be the space of discretely solenoidal functions. Let $\mathbf{u} \in \mathbf{J} \cap \mathbf{W}^{2,2}(\Omega)$ be the solution of

$$
(\nabla \mathbf{u}, \nabla \boldsymbol{\xi})-(p, \operatorname{div} \boldsymbol{\xi})=(\mathbf{f}, \boldsymbol{\xi}) \quad \forall \boldsymbol{\xi} \in \mathbf{W}_{0}^{1,2}(\Omega) .
$$

The solution $(\mathbf{U}, P) \in \mathbf{J}_{h} \times L_{h}$ of the discretized incompressible Stokes problem

$$
(\nabla \mathbf{U}, \nabla \boldsymbol{\xi})-(P, \operatorname{div} \boldsymbol{\xi})=(\mathbf{f}, \boldsymbol{\xi}) \quad \forall \boldsymbol{\xi} \in \mathbf{V}_{h}
$$

satisfies

$$
\|\mathbf{u}-\mathbf{U}\|_{\mathbf{L}^{2}}+h\|\mathbf{u}-\mathbf{U}\|_{\mathbf{W}^{1,2}} \leq C h^{2}\|\mathbf{u}\|_{\mathbf{W}^{2,2}} .
$$

We define $\mathbf{L}^{2}$-, resp., $\mathbf{W}_{0}^{1,2}$-orthogonal projections from (subspaces of) $\mathbf{J}$ to $\mathbf{J}_{h}$ via

$$
\left(\mathbf{z}-\mathbf{Q}_{h}^{0} \mathbf{z}, \boldsymbol{\xi}\right)=0 \quad \forall \boldsymbol{\xi} \in \mathbf{J}_{h}, \quad \text { resp., } \quad\left(\nabla\left[\mathbf{z}-\mathbf{Q}_{h}^{1} \mathbf{z}\right], \nabla \boldsymbol{\xi}\right)=0 \quad \forall \boldsymbol{\xi} \in \mathbf{J}_{h} .
$$

The following error estimates are standard, (see also [18]) $(i=0,1)$,

$$
\begin{aligned}
&\left\|\mathbf{z}-\mathbf{Q}_{h}^{i} \mathbf{z}\right\|_{\mathbf{L}^{2}}+h\left\|\nabla\left[\mathbf{z}-\mathbf{Q}_{h}^{i} \mathbf{z}\right]\right\|_{\mathbf{L}^{2}} \leq C h^{2}\|\mathbf{z}\|_{\mathbf{W}^{2,2}} \quad \forall \mathbf{z} \in \mathbf{J} \cap \mathbf{W}^{2,2}(\Omega), \\
&\left\|\mathbf{z}-\mathbf{Q}_{h}^{i} \mathbf{z}\right\|_{\mathbf{L}^{2}} \leq C h\|\mathbf{z}\|_{\mathbf{W}^{1,2}} \quad \forall \mathbf{z} \in \mathbf{J} \cap \mathbf{W}^{1,2}(\Omega) .
\end{aligned}
$$

The following compatibility condition of spaces

$$
V_{h} \cap L_{0}^{2}(\Omega) \subseteq L_{h}
$$

is needed to account for coupling effects in Scheme A given below.

We define the discrete Laplacian $\Delta_{h}: \mathbf{W}_{0}^{1,2}(\Omega) \rightarrow \mathbf{V}_{h}$ by

$$
\left(\Delta_{h} \mathbf{W}, \boldsymbol{\xi}\right)=-(\nabla \mathbf{W}, \nabla \boldsymbol{\xi}) \quad \forall \boldsymbol{\xi} \in \mathbf{V}_{h}
$$


For $\mathbf{W}=\mathcal{I}_{\mathbf{V}_{h}} \mathbf{w}$, where $\mathbf{w} \in \mathbf{W}_{0}^{1,2}(\Omega) \cap \mathbf{W}^{2,2}(\Omega)$, we obtain by inverse estimate and standard approximation results,

$$
\begin{aligned}
\left\|\Delta_{h} \mathbf{W}\right\|^{2} & =\left(\nabla \Delta_{h} \mathbf{W}, \nabla[\mathbf{W}-\mathbf{w}+\mathbf{w}]\right) \\
& \leq C h^{-1}\left\|\Delta_{h} \mathbf{W}\right\| C h\|\Delta \mathbf{w}\|+\left\|\Delta_{h} \mathbf{W}\right\|\|\Delta \mathbf{w}\| \leq C\left\|\Delta_{h} \mathbf{W}\right\|\|\Delta \mathbf{w}\| .
\end{aligned}
$$

The following discrete Sobolev interpolation inequality follows from 18, Lemma 4.4],

$$
\|\nabla \mathbf{W}\|_{\mathbf{L}^{4}} \leq C\|\nabla \mathbf{W}\|^{\frac{4-d}{d}}\left(\|\mathbf{W}\|+\left\|\Delta_{h} \mathbf{W}\right\|\right)^{\frac{d}{4}} \quad \forall \mathbf{W} \in \mathbf{V}_{h}
$$

Discrete magnetic field and multiplier spaces. We use edge elements to approximate solenoidal functions of Maxwell's equation, which only enforce continuity of tangential field components across inter-element boundaries. Below, we use Nédélec's first family of spaces (cf. 31]) for the unknown $\mathbf{B}: \Omega_{T} \rightarrow \mathbb{R}^{3}$. For every $K \in \mathcal{T}_{h}$ and $i \geq 1$, let $\mathcal{N}_{i}(K)=\left[\mathbf{P}_{i-1}(K)\right]^{3} \oplus \mathbf{D}_{i}(K)$, where $\mathbf{D}_{i}$ is given by the homogeneous polynomials $\mathbf{p}$ of degree $k$ that satisfy $\mathbf{p}(\mathbf{x}) \cdot \mathbf{x}=0$ on $K \in \mathcal{T}_{h}$. Consider then

$$
\begin{aligned}
\mathbf{C}_{h} & =\left\{\boldsymbol{\psi} \in \mathbf{H}(\text { curl }): \boldsymbol{\psi} \in \mathcal{N}_{i}(K) \quad \forall K \in \mathcal{T}_{h}\right\}, \\
S_{h} & =\left\{R \in W^{1,2}(\Omega) \cap L_{0}^{2}(\Omega): R \in \mathrm{P}_{i}(K) \quad \forall K \in \mathcal{T}_{h}\right\},
\end{aligned}
$$

where $\mathbf{C}_{h} \subset \mathbf{H}(\mathbf{c u r l} ; \Omega)$, and $S_{h} \subset W^{1,2}(\Omega) \cap L_{0}^{2}(\Omega)$. A well-known example is $i=1$, where $\mathbf{C}_{h}=\left\{\boldsymbol{\psi} \in \mathbf{H}(\mathbf{c u r l}): \boldsymbol{\psi}=\mathbf{a}_{K}+\mathbf{b}_{K} \times \mathbf{x}\right.$ on $\left.K \forall K \in \mathcal{T}_{h}\right\}$. The spaces $\left(\mathbf{C}_{h}, S_{h}\right)$ satisfy the following discrete inf-sup condition,

$$
\sup _{\boldsymbol{\psi}_{h} \in \mathbf{C}_{h}} \frac{(\nabla R, \boldsymbol{\psi})}{\|\boldsymbol{\psi}\|_{\mathbf{H}(\text { curl })}} \geq C\|\nabla R\|_{L^{2}} \quad \forall R \in S_{h} .
$$

We may define the interpolation $\boldsymbol{R}_{h}: \mathbf{H}(\mathbf{c u r l} ; \Omega) \rightarrow \mathbf{C}_{h}$, which satisfies for all $\mathbf{z} \in\left\{\overline{\mathbf{z}} \in \mathbf{W}^{1,2}: \operatorname{curl} \overline{\mathbf{z}} \in \mathbf{W}^{1,2}\right\}$ that ([31, Theorem 5.41])

$$
\left\|\mathbf{z}-\mathcal{R}_{h} \mathbf{z}\right\|_{\mathbf{L}^{2}}+\left\|\operatorname{curl}\left[\mathbf{z}-\mathcal{R}_{h} \mathbf{z}\right]\right\|_{\mathbf{L}^{2}} \leq C h\left(\|\mathbf{z}\|_{\mathbf{W}^{1,2}}+\|\operatorname{curl} \mathbf{z}\|_{\mathbf{W}^{1,2}}\right) .
$$

We introduce the discretely solenoidal function space

$$
\mathbf{X}_{h}:=\left\{\boldsymbol{\psi} \in \mathbf{C}_{h}:(\boldsymbol{\psi}, \nabla R)=0 \text { for all } R \in S_{h}\right\} \not \subset \mathbf{X} .
$$

We have the $L^{2}(\Omega)$-orthogonal discrete Helmholtz decomposition $\mathbf{C}_{h}=\mathbf{X}_{h} \oplus \nabla S_{h}$, as well as the discrete Poincaré-Friedrichs inequality $\|\boldsymbol{\psi}\|_{L^{2}} \leq C\|\operatorname{curl} \boldsymbol{\psi}\|_{L^{2}}$, for all $\boldsymbol{\psi} \in \mathbf{X}_{h}$; cf. [31, Theorem 4.7]. We introduce the $\mathbf{L}^{2}(\Omega)$-, resp., $\mathbf{H}(\mathbf{c u r l} ; \Omega)$ orthogonal projections to $\mathbf{X}_{h}$ via

$\left(\mathbf{z}-\mathbf{R}_{h}^{0} \mathbf{z}, \boldsymbol{\psi}\right)=0 \quad \forall \boldsymbol{\psi} \in \mathbf{X}_{h}, \quad$ resp., $\quad\left(\operatorname{curl}\left[\mathbf{z}-\mathbf{R}_{h}^{1} \mathbf{z}\right], \operatorname{curl} \boldsymbol{\psi}\right)=0 \quad \forall \boldsymbol{\psi} \in \mathbf{X}_{h}$.

The following estimates $(\ell=0,1)$ may be found in [5, Theorem 3.5] or [39, Lemma 3.4], and [20, Theorem 4.8],

$$
\begin{aligned}
& \left\|\mathbf{z}-\mathbf{R}_{h}^{\ell} \mathbf{z}\right\|_{\mathbf{L}^{2}}+\left\|\operatorname{curl}\left[\mathbf{z}-\mathbf{R}_{h}^{1} \mathbf{z}\right]\right\|_{\mathbf{L}^{2}} \\
& \quad \leq C h\left(\|\mathbf{z}\|_{\mathbf{W}^{1,2}}+\|\operatorname{curl} \mathbf{z}\|_{\mathbf{W}^{1,2}}\right) \quad \forall \mathbf{z} \in \mathbf{X} \cap \mathbf{W}^{2,2}(\Omega), \\
& \quad\left\|\mathbf{z}-\mathbf{R}_{h}^{\ell} \mathbf{z}\right\|_{\mathbf{L}^{2}} \leq C h^{\sigma}\|\operatorname{curl} \mathbf{z}\|_{\mathbf{L}^{2}} \quad \forall \mathbf{z} \in \mathbf{X}, \quad \text { for some } \sigma=\sigma(\Omega)>0 .
\end{aligned}
$$


Another link between the spaces $\mathbf{X}_{h}$ and $\mathbf{X}$ is accomplished by the Hodge mapping $\mathbf{H}: \mathbf{H}(\operatorname{curl} ; \Omega) \rightarrow \mathbf{X}$, such that

$$
\operatorname{curl} \mathbf{H}(\boldsymbol{\psi})=\operatorname{curl} \psi \quad \forall \boldsymbol{\psi} \in \mathbf{H}(\operatorname{curl} ; \Omega) .
$$

The following approximation property is shown in [20, Lemma 4.5],

$$
\|\mathbf{z}-\mathbf{H}(\mathbf{z})\|_{\mathbf{L}^{2}} \leq C h^{\frac{1}{2}+\sigma}\|\mathbf{c u r l} \mathbf{z}\|_{\mathbf{L}^{2}} \quad \forall \mathbf{z} \in \mathbf{X}_{h}, \quad \text { for some } \sigma=\sigma(\Omega)>0 .
$$

Lowest-order edge elements are divergence free inside each element. However, a nonzero divergence in distributional sense arises from discontinuities of the normal component at inter-element boundaries. Since $\mathbf{X}_{h} \not \subset \mathbf{X}$, the compactness of bounded sequences $\left\{\boldsymbol{\psi}_{h}\right\}_{h} \subset \mathbf{X}_{h} \subset \mathbf{H}(\mathbf{c u r l} ; \Omega)$ is not clear. The following (discrete) compactness property for discretely divergence-free vector fields generalizes the result by F. Kikuchi in [23]; cf. [20, Theorem 4.9].

Lemma 2.5. Let $\left\{\boldsymbol{\psi}_{h}\right\} \subset \mathbf{X}_{h}$ be a sequence of fields, which is uniformly bounded in $\mathbf{H}(\mathbf{c u r l} ; \Omega)$. Then there exists a subsequence $\left\{\boldsymbol{\psi}_{h^{\prime}}\right\}_{h^{\prime}}$ converging weakly in $\mathbf{H}(\mathbf{c u r l} ; \Omega)$, and strongly in $\mathbf{L}^{2}(\Omega)$ to a solenoidal function $\boldsymbol{\psi} \in \mathbf{X}$.

2.4. Time discretization. Given a time-step size $k>0$, and a sequence $\left\{\varphi^{j}\right\}$ in some Banach space $X$, we set $d_{t} \varphi^{j}:=k^{-1}\left\{\varphi^{j}-\varphi^{j-1}\right\}$ for $j \geq 1$. Note that $\left(d_{t} \varphi^{j}, \varphi^{j}\right)=\frac{1}{2} d_{t}\left\|\varphi^{j}\right\|^{2}+\frac{k}{2}\left\|d_{t} \varphi^{j}\right\|^{2}$, if $X$ is a Hilbert space. Piecewise constant interpolations of $\left\{\varphi^{j}\right\}$ are defined for $t \in\left[t_{j}, t_{j+1}\right)$, and $0 \leq j \leq J-1$ by

$$
\varphi^{+}(t):=\varphi^{j+1}, \quad \varphi^{-}(t):=\varphi^{j}
$$

and a piecewise affine interpolation on $\left[t_{j}, t_{j+1}\right)$ is defined by

$$
\varphi(t):=\frac{t-t_{j}}{k} \varphi^{j+1}+\frac{t_{j+1}-t}{k} \varphi^{j} .
$$

It follows that $\left\|\varphi^{+}-\varphi\right\|_{X}+\left\|\varphi^{-}-\varphi\right\|_{X} \leq 2 k\left\|d_{t} \varphi\right\|_{X}$.

\section{Scheme A: A fully discrete Scheme with Discrete maximum PRINCIPLE AND DISCRETE ENERGY LAW}

To obtain the energy law (1.5), we need to multiply $(1.1)_{1}$ with $\mathbf{u}$, and $(1.1)_{3}$ with $\frac{1}{2}|\mathbf{u}|^{2}$. We are motivated to construct a numerical scheme which shares properties (1.4) and (1.5) with the original problem (1.1)-(1.2); however, to use a test function which is a nonlinear function of the finite element velocity field does not seem to be a promising strategy. For this reason, the following reformulation is proposed in [30] in a related context:

$$
(\rho \mathbf{u})_{t}+\operatorname{div}(\rho \mathbf{u} \otimes \mathbf{u})=\frac{1}{2}\left\{\rho \mathbf{u}_{t}+[\rho \mathbf{u} \cdot \nabla] \mathbf{u}+(\rho \mathbf{u})_{t}+\operatorname{div}(\rho \mathbf{u} \otimes \mathbf{u})\right\} .
$$

If we take the dot product of the right-hand side with $\mathbf{u}$, integration in space yields $\frac{d}{d t} \int_{\Omega} \frac{\rho}{2}|\mathbf{u}|^{2} \mathrm{~d} \mathbf{x}$. We use this idea in the following implicit finite element discretization of (1.1)-(1.2). Below, we discuss the relevancy of additional stabilization terms added to allow for positive densities, and a discrete maximum principle. Let $\varphi_{+}:=\max \{0, \varphi\}$, and $(\cdot, \cdot)_{*}$ stands for either $(\cdot, \cdot)$, or $(\cdot, \cdot)_{h}$.

Scheme A: Let $\left(\mathbf{U}^{0}, \mathbf{B}^{0}, \rho^{0}\right) \in \mathbf{J}_{h} \times \mathbf{X}_{h} \times V_{h}$, such that $\bar{\rho}_{1} \leq \rho^{0} \leq \bar{\rho}_{2}$, and $\alpha, \beta_{1}, \beta_{2}, \beta_{3} \geq 0$. For every $n \geq 1$, find

$$
\left(\mathbf{U}^{n}, P^{n}, \mathbf{B}^{n}, R^{n}, \rho^{n}\right) \in \mathbf{J}_{h} \times L_{h} \times \mathbf{X}_{h} \times S_{h} \times V_{h}
$$


such that for all $(\chi, \mathbf{W}, \boldsymbol{\psi}) \in V_{h} \times \mathbf{V}_{h} \times \mathbf{C}_{h}$,

$$
\begin{aligned}
& \begin{array}{c}
(3.1) \quad\left(d_{t} \rho^{n}, \chi\right)_{h}+\left(\mathbf{U}^{n} \cdot \nabla \rho^{n}, \chi\right)+\frac{1}{2}\left(\left[\operatorname{div} \mathbf{U}^{n}\right] \rho^{n}, \chi\right)+\alpha h^{\alpha}\left(\nabla \rho^{n}, \nabla \chi\right)=0, \\
(3.2) \quad \frac{1}{2}\left\{\left(\rho_{+}^{n-1} d_{t} \mathbf{U}^{n}, \mathbf{W}\right)_{*}+\left(d_{t}\left[\rho_{+}^{n} \mathbf{U}^{n}\right], \mathbf{W}\right)_{*}+\left(\left[\rho^{n-1} \mathbf{U}^{n-1} \cdot \nabla\right] \mathbf{U}^{n}, \mathbf{W}\right)\right. \\
\left.-\left(\left[\rho^{n-1} \mathbf{U}^{n-1} \cdot \nabla\right] \mathbf{W}, \mathbf{U}^{n}\right)\right\}+\left(\eta^{n-1} \boldsymbol{D}\left(\mathbf{U}^{n}\right), \boldsymbol{D}(\mathbf{W})\right) \\
+\beta_{1} h^{-\beta_{1}}\left(\operatorname{div} \mathbf{U}^{n}, \operatorname{div} \mathbf{W}\right) \\
+\beta_{2} h^{\beta_{2}}\left(\nabla d_{t} \mathbf{U}^{n}, \nabla \mathbf{W}\right)+\beta_{3} h^{\beta_{3}}\left(\Delta_{h} \mathbf{U}^{n}, \Delta_{h} \mathbf{W}\right)-\left(P^{n}, \operatorname{div} \mathbf{W}\right) \\
\quad+\frac{1}{\bar{\mu}}\left(\mathbf{B}^{n-1} \times \operatorname{curl} \mathbf{B}^{n}, \mathbf{W}\right)=\left(\rho^{n-1} \mathbf{g}^{n}, \mathbf{W}\right), \\
(3.3) \quad\left(d_{t} \mathbf{B}^{n}, \boldsymbol{\psi}\right)+\frac{1}{\bar{\mu}}\left(\frac{1}{\xi^{n-1}} \operatorname{curl}^{n}, \operatorname{curl} \psi\right) \\
\quad-\left(\mathbf{U}^{n} \times \mathbf{B}^{n-1}, \operatorname{curl} \psi\right)-\left(\nabla R^{n}, \boldsymbol{\psi}\right)=0,
\end{array} \\
& \text { where } \eta^{n-1}:=\eta\left(\rho^{n-1}\right) \text { and } \xi^{n-1}:=\xi\left(\rho^{n-1}\right) .
\end{aligned}
$$

In fact, Scheme A comprises two discretization strategies, depending on the choice of $(\cdot, \cdot)_{*}$. We use several stabilizing terms in the scheme: the one lead by $\alpha$ allows for the $M$-matrix property of the stiffness matrix related to (3.1) to validate (3.6) below; it turns out that improved stability properties for $\left\{\mathbf{U}^{n}\right\}$ are needed for this purpose, which is the reason for the term lead by $\beta_{2} \geq 0$. Finally, strong $L^{p}$-convergence of $\left\{\operatorname{div} \mathbf{U}^{n}\right\}$ is needed to properly identify the limit in (3.1), which is the reason for the third stabilization term lead by $\beta_{1} \geq 0$; the convergence proof for the scheme below suggests the corresponding penalization parameter to depend on $h>0$ as well. In order to validate strong $L^{2}$-convergence of $\left\{\mathbf{U}^{n}\right\}$ in the case $d=3$ requires $\beta_{3}>0$ in the scheme; see Lemma 3.3. This term may be skipped in the two-dimensional setting $\Omega \subset \mathbb{R}^{2}$.

Note that in (3.3), we employ $R^{n} \in S_{h}$ as a Lagrange multiplier to enforce the discrete divergence free constraint for computed magnetic fields at finite scales $k, h>0$; the related term actually goes to zero for discretization parameters tending to zero.

We use numerical integration for the leading term in (3.1) to allow for a discrete maximum principle for computed densities, without any mesh constraint to hold. As will be clear from the following analysis (e.g., Step 1 in the proof of Lemma 3.3), this form of integration couples back with the one for the leading two terms in (3.2); as we will see later, stability, and monotonicity properties of the scheme are not affected by the choice of exact or numerical integration for the relevant terms in (3.2), while necessary compactness properties of velocity iterates of the scheme require numerical integration in (3.2) as well to be compatible with the formulation in (3.1); cf. Lemma 3.3.

The following result validates existence of solutions of Scheme A which satisfy a discrete energy identity $\left(\alpha, \beta_{1}, \beta_{2} \geq 0\right)$. Moreover, provided that meshes are of strongly acute type, and $\alpha, \beta_{1}, \beta_{2}>0$, we validate a discrete maximum principle for iterates $\left\{\rho^{n}\right\}_{n \geq 0}$ as well, i.e., the above scheme holds for existing iterates $\left\{\left(\mathbf{U}^{n}, P^{n}, \mathbf{B}^{n}, R^{n}, \rho^{n}\right)\right\}_{n=1}^{N}$, with $\rho_{+}^{n-1}$, resp., $\rho_{+}^{n}$ replaced by $\rho^{n-1}$, resp., $\rho^{n}$ in (3.2). 
Lemma 3.1. Let $\alpha, \beta_{1}, \beta_{2}, \beta_{3} \geq 0$, and $\sqrt{\beta_{2}} h^{\frac{\beta_{2}}{2}}\left\|\nabla \mathbf{U}^{0}\right\| \leq C$. For every $n \geq 1$, there exists a solution $\left(\mathbf{U}^{n}, P^{n}, \mathbf{B}^{n}, R^{n}, \rho^{n}\right) \in \mathbf{J}_{h} \times L_{h} \times \mathbf{X}_{h} \times S_{h} \times V_{h}$ of (3.1)(3.3), which satisfies

$$
\begin{aligned}
& \frac{1}{2} d_{t} {\left[\left\|\sqrt{\rho_{+}^{n}} \mathbf{U}^{n}\right\|_{*}^{2}+\beta_{2} h^{\beta_{2}}\left\|\nabla \mathbf{U}^{n}\right\|^{2}+\frac{1}{\bar{\mu}}\left\|\mathbf{B}^{n}\right\|^{2}\right] } \\
&+\left\|\sqrt{\eta^{n-1}} \mathbf{D}\left(\mathbf{U}^{n}\right)\right\|^{2}+\beta_{1} h^{-\beta_{1}}\left\|\operatorname{div} \mathbf{U}^{n}\right\|^{2}+\beta_{3} h^{\beta_{3}}\left\|\Delta_{h} \mathbf{U}^{n}\right\|^{2} \\
&+ \frac{1}{\bar{\mu}^{2}}\left\|\frac{1}{\sqrt{\xi^{n-1}}} \mathbf{c u r l}^{n}\right\|^{2} \\
&+ \frac{k}{2}\left[\left\|\sqrt{\rho_{+}^{n-1}} d_{t} \mathbf{U}^{n}\right\|_{*}^{2}+\beta_{2} h^{\beta_{2}}\left\|\nabla d_{t} \mathbf{U}^{n}\right\|^{2}+\left\|d_{t} \mathbf{B}^{n}\right\|^{2}\right] \\
&= \int_{\Omega} \rho^{n-1} \mathbf{g}^{n} \mathbf{U}^{n} \mathrm{~d} \mathbf{x}, \\
& \frac{1}{2} d_{t}\left\|\rho^{n}\right\|_{h}^{2}+\frac{k}{2}\left\|d_{t} \rho^{n}\right\|_{h}^{2}+\alpha h^{\alpha}\left\|\nabla \rho^{n}\right\|^{2}=0 .
\end{aligned}
$$

Moreover, if (2.8) holds, $\mathcal{T}_{h}$ is a strongly acute triangulation, and $0<\alpha, \beta_{2}$ such that $0<\alpha+\frac{\beta_{2}}{2}<\frac{6-d}{6}$, then

$$
0<\bar{\rho}_{1} \leq \rho^{n} \leq \bar{\rho}_{2}<\infty \quad(n \geq 1)
$$

provided $k, h>0$ are sufficiently small. As a consequence, $\rho_{+}^{n}$, resp., $\rho_{+}^{n-1}$ in (3.2) may be replaced by $\rho^{n}$, resp., $\rho^{n-1}$.

We use Brouwer's fixed point theorem to obtain solvability of (3.1)-(3.3) for every $n \geq 1$ in Step 1 of the following proof; we proceed by induction, assuming

$$
\max _{0 \leq \ell \leq n-1}\left\{\left\|\rho^{\ell}\right\|_{h}^{2}+\left\|\sqrt{\rho_{+}^{\ell}} \mathbf{U}^{\ell}\right\|_{*}^{2}+\beta_{2} h^{\beta_{2}}\left\|\nabla \mathbf{U}^{\ell}\right\|^{2}+\frac{1}{\bar{\mu}}\left\|\mathbf{B}^{\ell}\right\|^{2}\right\} \leq C,
$$

which then leads to the existence of $\left(\rho^{n}, \mathbf{U}^{n}, \mathbf{B}^{n}\right) \in V_{h} \times \mathbf{J}_{h} \times \mathbf{X}_{h}$. The bounds in Step 2 of the proof then justify (3.7) for $0 \leq \ell \leq n$. In Step 3, property (3.6) is verified, which requires the compatibility assumption (2.8) in part d) of this step of the proof, and uses the upper uniform bound $\max _{0 \leq n \leq N}\left\|\nabla \mathbf{U}^{n}\right\| \leq C h^{-\frac{\beta_{2}}{2}}$.

Proof. 1st Step: Solvability. For every $n \geq 1$, consider the continuous mapping $\mathcal{F}^{n}: V_{h} \times \mathbf{J}_{h} \times \mathbf{X}_{h} \rightarrow V_{h} \times \mathbf{J}_{h} \times \mathbf{X}_{h}$, which is defined as follows: For given $\left(\rho^{n-1}, \mathbf{U}^{n-1}, \mathbf{B}^{n-1}\right) \in V_{h} \times \mathbf{J}_{h} \times \mathbf{X}_{h}$ find $(\rho, \mathbf{U}, \mathbf{B}) \in V_{h} \times \mathbf{J}_{h} \times \mathbf{X}_{h}$ such that

$$
\begin{aligned}
\mathcal{F}^{n}([\rho, \mathbf{U}, \mathbf{B}],[\chi, \mathbf{W}, \boldsymbol{\psi}]) \\
\quad:=\left(\mathcal{F}_{1}^{n}([\rho, \mathbf{U}], \chi), \mathcal{F}_{2}^{n}([\rho, \mathbf{U}, \mathbf{B}], \mathbf{W}), \mathcal{F}_{3}^{n}([\mathbf{U}, \mathbf{B}], \boldsymbol{\psi})\right)^{\top}=\mathbf{0}
\end{aligned}
$$


for all $(\chi, \mathbf{W}, \boldsymbol{\psi}) \in V_{h} \times \mathbf{J}_{h} \times \mathbf{X}_{h}$, where

$$
\begin{aligned}
& \mathcal{F}_{1}^{n}([\rho, \mathbf{U}], \chi):= \frac{1}{k}\left(\rho-\rho^{n-1}, \chi\right)_{h}+(\mathbf{U} \cdot \nabla \rho, \chi) \\
&+\frac{1}{2}([\operatorname{div} \mathbf{U}] \rho, \chi)+\alpha h^{\alpha}(\nabla \rho, \nabla \chi)=0 \\
& \mathcal{F}_{2}^{n}([\rho, \mathbf{U}, \mathbf{B}], \mathbf{W}):=\frac{1}{2}\left\{\left(\frac{\rho_{+}^{n-1}}{k}\left[\mathbf{U}-\mathbf{U}^{n-1}\right], \mathbf{W}\right)_{*}+\frac{1}{k}\left(\left[\rho_{+} \mathbf{U}-\rho_{+}^{n-1} \mathbf{U}^{n-1}\right], \mathbf{W}\right)_{*}\right. \\
&\left.+\left(\left[\rho^{n-1} \mathbf{U}^{n-1} \cdot \nabla\right] \mathbf{U}, \mathbf{W}\right)-\left(\left[\rho^{n-1} \mathbf{U}^{n-1} \cdot \nabla\right] \mathbf{W}, \mathbf{U}\right)\right\} \\
&+\left(\eta^{n-1} \mathbf{D}(\mathbf{U}), \boldsymbol{D}(\mathbf{W})\right)+\beta_{1} h^{-\beta_{1}}\left(\operatorname{div} \mathbf{U}^{n}, \operatorname{div} \mathbf{W}\right) \\
&+\beta_{3} h^{\beta_{3}}\left(\Delta_{h} \mathbf{U}, \Delta_{h} \mathbf{W}\right) \\
&+\frac{\beta_{2} h^{\beta_{2}}}{k}\left(\nabla\left[\mathbf{U}-\mathbf{U}^{n-1}\right], \nabla \mathbf{W}\right)+\frac{1}{\bar{\mu}}\left(\mathbf{B}^{n-1} \times \mathbf{c u r l} \mathbf{B}, \mathbf{W}\right) \\
&-\left(\rho^{n-1} \mathbf{g}^{n}, \mathbf{W}\right), \\
& \mathcal{F}_{3}^{n}([\mathbf{U}, \mathbf{B}], \boldsymbol{\psi}): \frac{1}{k}\left(\mathbf{B}-\mathbf{B}^{n-1}, \boldsymbol{\psi}\right)+\frac{1}{\bar{\mu}}\left(\frac{1}{\xi^{n-1}} \mathbf{c u r l} \mathbf{B}, \mathbf{c u r l} \boldsymbol{\psi}\right) \\
&-\left(\mathbf{U} \times \mathbf{B}^{n-1}, \mathbf{c u r l} \psi\right) .
\end{aligned}
$$

By putting $[\chi, \mathbf{W}, \boldsymbol{\psi}]=[\rho, \mathbf{U}, \mathbf{B}]$ yields

$$
\begin{aligned}
\mathcal{F}^{n}( & {[\rho, \mathbf{U}, \mathbf{B}],[\rho, \mathbf{U}, \mathbf{B}]) } \\
\geq & \frac{1}{2 k}\left[\|\rho\|_{h}^{2}+\frac{1}{2}\left\|\sqrt{\rho_{+}^{n-1}} \mathbf{U}\right\|_{*}^{2}+\left\|\sqrt{\rho_{+}} \mathbf{U}\right\|_{*}^{2}+\frac{\beta_{2}}{2} h^{\beta_{2}}\|\nabla \mathbf{U}\|^{2}+\frac{1}{\bar{\mu}}\|\mathbf{B}\|^{2}\right] \\
& +\frac{\beta_{1}}{h^{\beta_{1}}}\|\operatorname{div} \mathbf{U}\|^{2} \\
& +\alpha h^{\alpha}\|\nabla \rho\|^{2}+\left\|\sqrt{\eta^{n-1}} \boldsymbol{D}(\mathbf{U})\right\|^{2}+\beta_{3} h^{\beta_{3}}\left\|\Delta_{h} \mathbf{U}\right\|^{2}+\frac{1}{\bar{\mu}^{2}}\left\|\frac{1}{\sqrt{\xi^{n-1}}} \mathbf{c u r l} \mathbf{B}\right\|^{2} \\
& -\frac{1}{4 k}\left[2\left\|\rho^{n-1}\right\|_{h}^{2}+2\left\|\sqrt{\rho_{+}^{n-1}} \mathbf{U}^{n-1}\right\|_{*}^{2}+\beta_{2} h^{\beta_{2}}\left\|\nabla \mathbf{U}^{n-1}\right\|^{2}+\frac{1}{\bar{\mu}}\left\|\mathbf{B}^{n-1}\right\|^{2}\right] .
\end{aligned}
$$

Because of (3.7), the modulus of the negative terms is finite; hence, provided that $(\rho, \mathbf{U}, \mathbf{B}) \in V_{h} \times \mathbf{J}_{h} \times \mathbf{X}_{h}$ satisfy for a sufficiently large, finite constant $\bar{C}_{n} \equiv$ $\bar{C}_{n}\left(\rho^{n-1}, \mathbf{U}^{n-1}, \mathbf{B}^{n-1}\right)>0$ that

$$
\min \{\|\rho\|,\|\mathbf{U}\|,\|\mathbf{B}\|\} \geq \bar{C}_{n}
$$

we always have $\mathcal{F}^{n}([\rho, \mathbf{U}, \mathbf{B}],[\rho, \mathbf{U}, \mathbf{B}]) \geq 0$. A consequence of Brouwer's fixed point theorem [35, p. 37] now implies existence of $\left(\rho^{n}, \mathbf{U}^{n}, \mathbf{B}^{n}\right) \in V_{h} \times \mathbf{J}_{h} \times \mathbf{X}_{h}$ which solves Scheme A for every $n \geq 1$.

Existence of corresponding $\left(P^{n}, R^{n}\right) \in L_{h} \times S_{h}$ is then a consequence of (2.5), (2.11). 
2nd Step: Discrete energy identity. Choose $\boldsymbol{\psi}=\frac{1}{\mu} \mathbf{B}^{n}$ in (3.3), $\mathbf{W}=\mathbf{U}^{n}$ in (3.2), and use

$$
\begin{aligned}
& \left(\rho_{+}^{n-1} d_{t} \mathbf{U}^{n}, \mathbf{U}^{n}\right)_{*}+\left(d_{t}\left[\rho_{+}^{n} \mathbf{U}^{n}\right], \mathbf{U}^{n}\right)_{*} \\
= & \frac{1}{k}\left(\rho_{+}^{n}\left|\mathbf{U}^{n}\right|^{2}+\rho_{+}^{n-1}\left|\mathbf{U}^{n}\right|^{2}-2 \rho_{+}^{n-1}\left\langle\mathbf{U}^{n-1}, \mathbf{U}^{n}, 1\right\rangle\right)_{*} \\
= & \frac{1}{k}\left(\rho_{+}^{n}\left|\mathbf{U}^{n}\right|^{2}+\rho_{+}^{n-1}\left|\mathbf{U}^{n}\right|^{2}-\rho_{+}^{n-1}\left\langle\mathbf{U}^{n-1}, \mathbf{U}^{n}\right\rangle\right. \\
& \left.\quad-\rho_{+}^{n-1}\left|\mathbf{U}^{n-1}\right|^{2}+\rho_{+}^{n-1}\left\langle\mathbf{U}^{n-1}, \mathbf{U}^{n-1}-\mathbf{U}^{n}\right\rangle, 1\right)_{*} \\
= & \frac{1}{k}\left(\rho_{+}^{n}\left|\mathbf{U}^{n}\right|^{2}+\rho_{+}^{n-1}\left|\mathbf{U}^{n}\right|^{2}-\rho_{+}^{n-1}\left\{\left|\mathbf{U}^{n}\right|^{2}+\left|\mathbf{U}^{n-1}\right|^{2}-k^{2}\left|d_{t} \mathbf{U}^{n}\right|^{2}\right\}, 1\right)_{*} \\
= & \left(d_{t}\left[\rho_{+}^{n}\left|\mathbf{U}^{n}\right|^{2}\right]+k \rho_{+}^{n-1}\left|d_{t} \mathbf{U}^{n}\right|^{2}, 1\right)_{*} .
\end{aligned}
$$

Adding both resulting equations then yields (3.4).

3rd Step: Discrete maximum principle. Fix $n \geq 1$. We verify the $M$-matrix property of the sub-system matrix related to (3.1), for given $\mathbf{U}^{n}: \Omega \rightarrow \mathbb{R}^{d}$. Let $\left\{\varphi_{\mathbf{x}_{\ell}}\right\}_{\ell=1}^{L}$ be the canonical basis of $V_{h}$. We employ vectors $\mathbf{u}^{n}=\left(\mathbf{u}_{\ell}^{n}\right)_{\ell=1}^{L}$, with $\mathbf{U}^{n}=\sum_{\ell=1}^{L} \mathbf{u}_{\ell}^{n} \varphi_{\mathbf{x}_{\ell}}$. We use the mass matrix $M:=\left\{m_{\ell \ell^{\prime}}\right\}_{\ell, \ell^{\prime}=1}^{L}$, stiffness matrix $K:=\left\{k_{\ell \ell^{\prime}}\right\}_{\ell, \ell^{\prime}=1}^{L}$, and $C\left(\mathbf{U}^{n}\right):=\left\{c_{\ell \ell^{\prime}}^{n}\right\}_{\ell, \ell^{\prime}=1}^{L}$, with

$$
\begin{aligned}
m_{\ell \ell^{\prime}} & :=\left(\varphi_{\mathbf{x}_{\ell}}, \varphi_{\mathbf{x}_{\ell^{\prime}}}\right)_{h}, \quad k_{\ell \ell^{\prime}}:=\left(\nabla \varphi_{\mathbf{x}_{\ell}}, \nabla \varphi_{\mathbf{x}_{\ell^{\prime}}}\right), \\
c_{\ell \ell^{\prime}}^{n} & :=\left(\mathbf{U}^{n} \cdot \nabla \varphi_{\mathbf{x}_{\ell}}, \varphi_{\mathbf{x}_{\ell^{\prime}}}\right)+\frac{1}{2}\left(\left[\operatorname{div} \mathbf{U}^{n}\right] \varphi_{\mathbf{x}_{\ell}}, \varphi_{\mathbf{x}_{\ell^{\prime}}}\right),
\end{aligned}
$$

to restate (3.1) as follows: $\mathcal{A}^{n} \mathbf{u}^{n}=\mathbf{g}^{n}$, with

$$
\mathcal{A}^{n}:=\frac{1}{k} M+C\left(\mathbf{U}^{n}\right)+\alpha h^{\alpha} K \in \mathbb{R}^{L \times L}, \quad g_{\ell}^{n}:=\frac{1}{k}\left(\rho^{n-1}, \varphi_{\mathbf{x}_{\ell}}\right) \quad(1 \leq \ell \leq L) .
$$

The following three steps establish that $\mathcal{A}^{n}$ is an $M$-matrix.

a) Nonpositivity of off-diagonal entries of $\boldsymbol{A}^{n}$. Let $\mathcal{T}_{h}$ be strongly acute; then there exists $C_{\theta_{0}}>0$ such that $k_{\ell \ell^{\prime}} \leq-C_{\theta_{0}} h^{d-2}<0$ uniformly in $h>0$, for any pair of adjacent nodes. Moreover, $m_{\ell \ell^{\prime}}=0$. The remaining entries are bounded by inverse estimates, local support property of basis functions $\left\{\varphi_{\mathbf{x}_{\ell}}\right\}_{\ell=1}^{L}$, and (3.4),

$$
\left|c_{\ell \ell^{\prime}}^{n}\right| \leq C h^{d-1}\left\|\mathbf{U}^{n}\right\|_{\mathbf{L}^{\infty}} \leq C h^{d-1-\frac{d}{6}}\left\|\mathbf{U}^{n}\right\|_{\mathbf{L}^{6}} \leq C h^{d-1-\frac{d}{6}-\frac{\beta_{2}}{2}} .
$$

In order to verify nonpositivity of $h^{\alpha} k_{\ell \ell^{\prime}}+c_{\ell \ell^{\prime}}\left(\mathbf{U}^{n}\right)$ for neighboring $\ell \neq \ell^{\prime}$, we have to make sure that

$$
\alpha+d-2<d-1-\frac{d}{6}-\frac{\beta_{2}}{2} \quad \Leftrightarrow \quad \alpha+\frac{\beta_{2}}{2}<\frac{6-d}{6} .
$$

b) Strict positivity of diagonal entries of $\mathcal{A}^{n}$. By evidence, $m_{\ell \ell} \geq c_{\theta_{0}} h^{d}$, and $k_{\ell \ell} \geq c_{\theta_{0}} h^{d-2}$, for some $c_{\theta_{0}}>0$. Similar to a), in order to make sure that $\frac{1}{k} m_{\ell \ell}+$ $c_{\ell \ell}\left(\mathbf{U}^{n}\right)+\alpha h^{\alpha} k_{\ell \ell}>0$, a dimensionality argument leads to $\alpha+d-2<d-1-\frac{d}{6}-\frac{\beta_{2}}{2}$, and we may conclude as above.

c) $\mathcal{A}^{n}$ is strictly diagonal dominant. We again use the fact that the number of neighboring nodes $\mathbf{x}_{\ell^{\prime}} \in \mathcal{N}_{h}$ for each $\mathbf{x}_{\ell}$ is bounded independently of $h>0$. Hence, there exists a constant $\bar{C} \equiv \bar{C}\left(\#\left\{\ell^{\prime}:\left(\nabla \varphi_{\mathbf{x}_{\ell}^{\prime}}, \nabla \varphi_{\mathbf{x}_{\ell}}\right) \neq 0\right\}\right)>0$, such that for 
sufficiently small $h>0$, and $\tilde{K}=\bigcup_{\overline{K^{\prime}} \cap \bar{K} \neq \emptyset} K^{\prime}$, for every $K \in \mathcal{T}_{h}$,

$$
\frac{1}{k} \bar{C} h^{d}+\alpha \bar{C} h^{\alpha+d-2}-\bar{C} h^{\frac{5 d}{6}-1-\frac{\beta_{2}}{2}}>0,
$$

which again implies $\alpha+\frac{\beta_{2}}{2}<\frac{6-d}{6}$.

Hence, from a)-c) we may conclude that $\mathcal{A}^{n}$ is an $M$-matrix, which then implies nonnegativity of $\left\{\rho^{n}\right\}_{n=1}^{N}$, by induction.

d) Boundedness of $\bar{\rho}_{1} \leq \rho^{n} \leq \bar{\rho}_{2}$. Let $\bar{\rho}^{n}:=\rho^{n}-\bar{\rho}_{2}$. Then, $\bar{\rho}^{n-1} \leq 0$, and we obtain for $\mathbf{U}^{n} \in \mathbf{J}_{h}$ :

$$
\begin{aligned}
\frac{1}{k}\left(\bar{\rho}^{n}, \chi\right)_{h}+\left(\mathbf{U}^{n}\right. & \left.\cdot \nabla \bar{\rho}^{n}, \chi\right)+\frac{1}{2}\left(\left[\operatorname{div} \mathbf{U}^{n}\right] \bar{\rho}^{n}, \chi\right)+\alpha h^{\alpha}\left(\nabla \bar{\rho}^{n}, \nabla \chi\right) \\
& =\frac{1}{k}\left(\bar{\rho}^{n-1}, \chi\right)_{h} \quad \forall \chi \in V_{h} .
\end{aligned}
$$

Here, we employ (2.8) to validate $\left(\operatorname{div} \mathbf{U}^{n}, \chi\right)=0$ for all $\chi \in V_{h}$. Let $[\chi]^{+}:=$ $\mathcal{I}_{V_{h}}(\max \{0, \chi\})$, and $[\chi]^{-}:=\mathcal{I}_{V_{h}}(\min \{0, \Phi\})$ for $\Phi \in V_{h}$. We wish to validate

$$
\begin{aligned}
\frac{1}{k}\left\|[\chi]^{+}\right\|_{h}^{2} & +h^{\alpha}\left\|\nabla[\chi]^{+}\right\|^{2} \leq \frac{1}{k}\left([\chi]^{+}, \chi\right)_{h}+\alpha h^{\alpha}\left(\nabla[\chi]^{+}, \nabla \chi\right) \\
& +\left(\mathbf{U}^{n} \cdot \nabla \chi,[\chi]^{+}\right)+\frac{1}{2}\left(\left[\operatorname{div} \mathbf{U}^{n}\right] \chi,[\chi]^{+}\right) \quad \forall \chi \in V_{h} .
\end{aligned}
$$

This result follows from $\left(\mathbf{U}^{n} \cdot \nabla[\chi]^{+},[\chi]^{+}\right)+\frac{1}{2}\left(\left[\operatorname{div} \mathbf{U}^{n}\right][\chi]^{+},[\chi]^{+}\right)=0$, and $\mathcal{A}^{n}=$ $\left\{a_{\ell \ell^{\prime}}^{n}\right\}_{\ell, \ell^{\prime}=1}^{L}$ being an $M$-matrix, for $h>0$ sufficiently small, such that

$$
\begin{aligned}
\frac{1}{k}\left(\chi-[\chi]^{+},[\chi]^{+}\right)_{h}+\left(\mathbf{U}^{n} \cdot \nabla\left\{\chi-[\chi]^{+}\right\},[\chi]^{+}\right) \\
+\frac{1}{2}\left(\left[\operatorname{div} \mathbf{U}^{n}\right]\left\{\chi-[\chi]^{+}\right\},[\chi]^{+}\right)+\alpha h^{\alpha}\left(\nabla\left\{\chi-[\chi]^{+}\right\}, \nabla[\chi]^{+}\right) \\
\geq \frac{1}{k}\left([\chi]^{-},[\chi]^{+}\right)_{h}+\left(\mathbf{U}^{n} \cdot \nabla[\chi]^{-},[\chi]^{+}\right) \\
\quad+\frac{1}{2}\left(\left[\operatorname{div} \mathbf{U}^{n}\right][\chi]^{-},[\chi]^{+}\right)+\alpha h^{\alpha}\left(\nabla[\chi]^{-}, \nabla[\chi]^{+}\right) \\
\geq \sum_{1 \leq \ell, \ell^{\prime} \leq L} a_{\ell \ell^{\prime}}^{n}[\chi]^{+}\left(\mathbf{x}_{\ell}\right)[\chi]^{-}\left(\mathbf{x}_{\ell^{\prime}}\right) \geq 0 .
\end{aligned}
$$

We may also verify (3.9), with negative sign in front of the last term. Now, putting $\chi=\left[\bar{\rho}^{n}\right]^{+}$in (3.8), noting again that $\bar{\rho}^{n}=\left[\bar{\rho}^{n}\right]^{+}+\left[\bar{\rho}^{n}\right]^{-}$, we obtain by (3.9) that

$$
\frac{1}{k}\left\|\left[\bar{\rho}^{n}\right]^{+}\right\|_{h}^{2}+\left\|\nabla\left[\bar{\rho}^{n}\right]^{+}\right\|^{2} \leq \frac{1}{k}\left(\bar{\rho}^{n-1},\left[\bar{\rho}^{n}\right]^{+}\right)_{h} \leq 0,
$$

and hence $\rho^{n} \leq \bar{\rho}_{2}$, for all $n \geq 0$. The lower bound follows immediately from the $M$-matrix property of the system matrix for (3.8), where $\bar{\rho}^{n}$ is substituted by $\underline{\rho}^{n}:=\rho^{n}-\bar{\rho}_{1}$.

Let $\left\{\left(\mathbf{U}^{n}, P^{n}, \mathbf{B}^{n}, R^{n}, \rho^{n}\right)\right\}_{n=1}^{N} \subset \mathbf{J}_{h} \times L_{h} \times \mathbf{X}_{h} \times S_{h} \times V_{h}$ be a solution of Scheme A, such that (3.6) holds; consider the continuous interpolants in time $\{(\mathcal{U}(t, \cdot), \wp(t, \cdot), \mathcal{B}(t, \cdot), R(t, \cdot), \sigma(t, \cdot))\}_{0 \leq t \leq T}$ as defined in Section 2.4 and depending on $k, h>0$ in particular. This pentuple solves for every $(\mathbf{W}, \boldsymbol{\psi}, \chi) \in \mathbf{J}_{h} \times \mathbf{X}_{h} \times$ 
$V_{h}$, and $0 \leq t \leq T$

$$
\begin{gathered}
\left(\sigma_{t}, \chi\right)_{h}+\left(\mathcal{U}^{+} \cdot \nabla \sigma^{+}, \chi\right)+\frac{1}{2}\left(\left[\operatorname{div} \mathcal{U}^{+}\right] \sigma^{+}, \chi\right)+\alpha h^{\alpha}\left(\nabla \sigma^{+}, \nabla \chi\right)=0 \\
\frac{1}{2}\left\{\left(\sigma^{-} \mathcal{U}_{t}, \mathbf{W}\right)_{*}+\left([\sigma \mathcal{U}]_{t}, \mathbf{W}\right)_{*}+\left(\left[\sigma^{-} \mathcal{U}^{-} \cdot \nabla\right] \mathcal{U}^{+}, \mathbf{W}\right)\right. \\
\left.-\left(\left[\sigma^{-} \mathcal{U}^{-} \cdot \nabla\right] \mathbf{W}, \mathcal{U}^{+}\right)\right\} \\
+\left(\eta^{-} \boldsymbol{D}\left(\mathcal{U}^{+}\right), \boldsymbol{D}(\mathbf{W})\right)+\beta_{1} h^{-\beta_{1}}\left(\operatorname{div} \mathcal{U}^{+}, \operatorname{div} \mathbf{W}\right)+\beta_{2} h^{\beta_{2}}\left(\nabla \mathcal{U}_{t}, \nabla \mathbf{W}\right) \\
+\beta_{3} h^{\beta_{3}}\left(\Delta_{h} \mathcal{U}^{+}, \Delta_{h} \mathbf{W}\right)+\frac{1}{\bar{\mu}}\left(\boldsymbol{B}^{-} \times \mathbf{c u r l} \mathcal{B}^{+}, \mathbf{W}\right)=\left(\sigma^{-} \mathbf{g}^{+}, \mathbf{W}\right) \\
\left(\boldsymbol{B}_{t}, \boldsymbol{\psi}\right)+\frac{1}{\bar{\mu}}\left(\frac{1}{\xi^{-}} \operatorname{curl} \mathcal{B}^{+}, \operatorname{curl} \boldsymbol{\psi}\right)-\left(\boldsymbol{U}^{+} \times \mathcal{B}^{-}, \operatorname{curl} \boldsymbol{\psi}\right)=0 .
\end{gathered}
$$

The uniform bounds (3.4) and (3.6) allow for convergent subsequences, and functions

$$
\begin{gathered}
\mathbf{u} \in L^{\infty}(0, T ; \mathbf{H}) \cap L^{2}(0, T ; \mathbf{J}), \quad \mathbf{b} \in L^{\infty}(0, T ; \mathbf{H}) \cap L^{2}(0, T ; \mathbf{X}), \\
\rho \in L^{\infty}\left(0, T ; L^{\infty}(\Omega)\right)
\end{gathered}
$$

such that for $k, h \rightarrow 0$,

$$
\begin{aligned}
\boldsymbol{U}^{ \pm}, \boldsymbol{U} \stackrel{*}{\rightarrow} \mathbf{u} & \text { in } L^{\infty}\left(0, T ; \mathbf{L}^{2}\right), \\
\mathcal{U}^{ \pm} \rightarrow \mathbf{u} & \text { in } L^{2}\left(0, T ; \mathbf{W}^{1,2}\right), \\
\operatorname{div} \mathcal{U}^{ \pm} \rightarrow 0 & \text { in } L^{2}\left(0, T ; L^{2}(\Omega)\right) \quad\left(\text { for } \beta_{1}>0\right), \\
\mathcal{B}^{ \pm}, \mathcal{B} \stackrel{*}{\rightarrow} \mathbf{b} & \text { in } L^{\infty}\left(0, T ; \mathbf{L}^{2}\right), \\
\mathcal{B}^{ \pm} \rightarrow \mathbf{b} & \text { in } L^{2}(0, T ; \mathbf{H}(\mathbf{c u r l})), \\
\sigma^{ \pm}, \sigma \stackrel{*}{*} \rho & \text { in } L^{\infty}\left(0, T ; L^{\infty}\right) .
\end{aligned}
$$

The incompressibility of $\mathbf{u}$ as stated in $(3.12)$ follows from $\int_{0}^{T}\left(\operatorname{div} \mathbf{U}^{+}, \Pi\right) \mathrm{d} s=0$ for all $\Pi(t, \cdot) \in L_{h}$ : on putting $\Pi(t, \cdot)=I_{L_{h}} \chi(t, \cdot) \in L_{h}$ for $\chi \in C^{\infty}\left([0, T], C^{\infty}(\Omega)\right)$, and tending $k, h \rightarrow 0$ validates the assertion; strong convergence as stated in (3.13) 3 then follows from weak and norm convergence. Also, we use Lemma 2.5 to make sure in assertion (3.12) 2 that $\mathbf{b}$ is divergence-free. Moreover, from (3.4) and (3.6) we may conclude that sequences $\{\mathcal{U}\}$, resp., $\left\{\mathcal{U}^{ \pm}\right\}$, as well as $\{\mathcal{B}\}$, resp., $\left\{\mathcal{B}^{ \pm}\right\}$, and $\{\sigma\}$, resp., $\left\{\sigma^{ \pm}\right\}$converge to the same limit as $k, h \rightarrow 0$, since, for example,

$$
\begin{aligned}
\left\|\mathcal{U}-\mathcal{U}^{+}\right\|_{L^{2}\left(0, T ; \mathbf{L}^{2}\right)}^{2} & =\sum_{n=1}^{N}\left\|\mathbf{U}^{n}-\mathbf{U}^{n-1}\right\|_{\mathbf{L}^{2}}^{2} \int_{t_{n-1}}^{t_{n}}\left(\frac{s-t_{n}}{k}\right)^{2} \mathrm{~d} s \\
& =\frac{k^{3}}{3} \sum_{n=1}^{N}\left\|d_{t} \mathbf{U}^{n}\right\|_{\mathbf{L}^{2}}^{2},
\end{aligned}
$$

which tends to zero for $k \rightarrow 0$.

We now detail passing to the limit in equation $(3.11)_{1}$, under the assumptions that

$$
\rho^{0} \rightarrow \rho_{0} \quad \text { in } L^{2}(\Omega) \quad \text { and } \quad \mathcal{U}^{+} \rightarrow \mathbf{u} \quad \text { in } L^{2}\left(0, T ; \mathbf{L}^{2}(\Omega)\right)
$$


for $k, h \rightarrow 0$ hold. We remark that $(3.15)_{2}$ will be shown to hold in Lemma 3.3. Let $\Xi(t, \cdot)=\mathcal{I}_{V_{h}} \chi(t, \cdot)$ for every $t \in[0, T)$, and all $\chi \in C_{0}^{\infty}\left([0, T) ; C_{0}^{\infty}(\Omega)\right)$. Because of

$$
\left(\mathcal{U}^{+} \cdot \nabla \sigma^{+}, \Xi\right)=\left(\operatorname{div}\left[\sigma^{+} \mathcal{U}^{+}\right], \Xi\right)-\left(\left[\operatorname{div} \mathcal{U}^{+}\right] \sigma^{+}, \Xi\right)
$$

we may recast (3.11) 1 in the form

$$
\left(\sigma_{t}, \Xi\right)_{h}-\left(\mathcal{U}^{+} \sigma^{+}, \nabla \Xi\right)-\frac{1}{2}\left(\left[\operatorname{div} \mathcal{U}^{+}\right] \sigma^{+}, \Xi\right)+\alpha h^{\alpha}\left(\nabla \sigma^{+}, \nabla \Xi\right)=0 .
$$

We integrate over $[0, T]$, and identify limits term by term. For the first term, we apply integration by parts, and employ (2.4), the $W^{1,2}$-stability of $\mathcal{I}_{V_{h}}$, and related convergence properties, and $(3.13)_{6} 6$ to conclude that

$$
\begin{aligned}
& \left|\int_{0}^{T}\left(\sigma, \Xi_{t}\right)_{h}-\left(\sigma, \Xi_{t}\right) \mathrm{d} s\right| \leq C h\|\sigma\|_{L^{2}\left(0, T ; L^{2}\right)}\left\|\Xi_{t}\right\|_{L^{2}\left(0, T ; W^{1,2}\right)} \\
& \quad \leq C h\left\{\left\|(\chi-\Xi)_{t}\right\|_{L^{2}\left(0, T ; W^{1,2}\right)}+\left\|\chi_{t}\right\|_{L^{2}\left(0, T ; W^{1,2}\right)}\right\} \rightarrow 0 \quad(h \rightarrow 0) .
\end{aligned}
$$

Because of $(3.15)_{1},(3.13)_{6}$, we may then conclude that

$$
-\int_{0}^{T}\left(\sigma_{t}, \Xi\right)_{h} \mathrm{~d} s \rightarrow \int_{0}^{T}\left(\rho, \chi_{t}\right) \mathrm{d} s+\left(\rho_{0}, \chi(0, \cdot)\right) \quad(k, h \rightarrow 0) .
$$

The result

$$
\int_{0}^{T}\left(\mathcal{U}^{+} \sigma^{+}, \nabla \Xi\right) \mathrm{d} s \rightarrow \int_{0}^{T}(\mathbf{u} \rho, \nabla \chi) \mathrm{d} s \quad(k, h \rightarrow 0)
$$

easily follows from $(3.15)_{2},(3.13)_{6}$. We proceed similarly with the third term in (3.16), by exploiting (3.13) 3 , in particular.

For the last term in (3.16), we use (3.5) to conclude that

$$
\alpha h^{\alpha}\left|\int_{0}^{T}\left(\nabla \sigma^{+}, \nabla \Xi\right) \mathrm{d} s\right| \leq C \sqrt{\alpha} h^{\frac{\alpha}{2}}\|\nabla \Xi\|_{L^{2}\left(0, T, \mathbf{L}^{2}\right)} \rightarrow 0 \quad(h \rightarrow 0) .
$$

Putting things together then validates that $\rho$ is a weak solution of $(1.1)_{3}$.

As a next step, in order to pass to the limit in terms of $(3.11)_{2}$, we need to make sure that densities converge strongly in $L^{p}$; in [38, Theorem 5.1], a general compactness result is shown for solutions of a discrete version of (1.1) 3 , where a discontinuous Galerkin method is used. We adapt the arguments given there to our problem, and also summarize the above results in the following:

Lemma 3.2. Fix $T>0$, suppose (2.8), and that $0<\alpha, \beta_{2}$ are such that $0<$ $\alpha+\frac{\beta_{2}}{2}<\frac{6-d}{6}$. Let $\{\mathcal{U}\}_{k, h} \subset L^{2}\left(0, T ; \mathbf{W}_{0}^{1,2}(\Omega)\right)$, and $\{\sigma\}_{k, h} \subset L^{\infty}\left(0, T ; L^{2}(\Omega)\right)$ be given, such that for all $\chi \in V_{h}$, and $n \geq 1$,

$$
\left(d_{t} \rho^{n}, \chi\right)_{h}+\left(\mathbf{U}^{n} \cdot \nabla \rho^{n}, \chi\right)+\frac{1}{2}\left(\left[\operatorname{div} \mathbf{U}^{n}\right] \rho^{n}, \chi\right)+\alpha h^{\alpha}\left(\nabla \rho^{n}, \nabla \chi\right)=0,
$$

where $\rho^{0} \in V_{h}$, such that $h^{\delta}\left\|\nabla \rho^{0}\right\| \leq C$, for some $0 \leq \delta<1$. Suppose that for $k, h \rightarrow 0$ we have $\rho^{0} \rightarrow \rho_{0}$ in $L^{2}(\Omega)$, as well as

$$
\mathcal{U}^{+} \rightarrow \mathbf{u} \quad \text { in } L^{2}\left(0, T ; \mathbf{L}^{2}(\Omega)\right), \quad \sigma^{+} \stackrel{*}{\rightarrow} \rho \quad \text { in } L^{\infty}\left(0, T ; L^{2}(\Omega)\right),
$$

and $\operatorname{div} \mathcal{U}^{+} \rightarrow 0$ in $L^{1}\left(0, T ; L^{2}(\Omega)\right)$. Then, $\rho: \Omega_{T} \rightarrow \mathbb{R}$ is a unique weak solution of (1.1) 3 , which satisfies properties (1.1)-(1.2) of Lemma 2.3, and $\sigma \rightarrow \rho$ in $L^{2}\left(0, T ; L^{2}(\Omega)\right)$ for $k, h \rightarrow 0$. 
Proof. We verify that $\lim _{k, h \rightarrow 0}\|\sigma\|_{L^{2}\left(0, T ; L^{2}(\Omega)\right)}=\|\rho\|_{L^{2}\left(0, T ; L^{2}(\Omega)\right)}$; since weak convergence and convergence of norms implies strong convergence in reflexive Banach spaces, this settles the result of the lemma.

For this purpose, we use the identity (2.2) for $\rho: \Omega_{T} \rightarrow \mathbb{R}$ with $\beta(s)=\frac{1}{2} s^{2}$, and $\rho^{0} \rightarrow \rho_{0}$ in $L^{2}(\Omega)$ to obtain

$$
\frac{1}{2}\|\rho(t, \cdot)\|^{2}=\frac{1}{2}\left\|\rho_{0}\right\|^{2}=\frac{1}{2} \liminf _{h \rightarrow 0}\left\|\rho^{0}\right\|^{2},
$$

and after integration over the time interval $[0, T]$, by using (2.4) together with $h^{\delta}\left\|\nabla \rho^{0}\right\| \leq C$ for $0 \leq \delta<1$, we get

$$
\frac{1}{2}\|\rho\|_{L^{2}\left(0, T ; L^{2}\right)}^{2}=\frac{T}{2} \liminf _{h \rightarrow 0}\left\|\rho^{0}\right\|_{L^{2}}^{2}=\frac{T}{2} \liminf _{h \rightarrow 0}\left\|\rho^{0}\right\|_{h}^{2} .
$$

Once we show that

$$
\begin{aligned}
\liminf _{h \rightarrow 0} & \frac{T}{2}\left\|\rho^{0}\right\|_{h}^{2} \\
& \geq \liminf _{k, h \rightarrow 0}\left(\frac{1}{2}\|\sigma\|_{L^{2}\left(0, T ; L^{2}\right)}^{2}+\int_{0}^{T} \int_{0}^{s}\left[\frac{k}{2}\left\|\sigma_{t}\right\|_{h}^{2}+\alpha h^{\alpha}\left\|\nabla \sigma^{+}\right\|^{2}\right] \mathrm{d} t \mathrm{~d} s\right),
\end{aligned}
$$

we deduce by lower semicontinuity property of norms with respect to weak-star convergence in $L^{\infty}\left(0, T ; L^{2}(\Omega)\right)$, and $\sigma \stackrel{*}{\rightarrow} \rho$ in $L^{\infty}\left(0, T ; L^{2}(\Omega)\right)$ that

$$
\|\rho\|_{L^{2}\left(0, T ; L^{2}\right)}^{2} \leq \liminf _{k, h \rightarrow 0}\|\sigma\|_{L^{2}\left(0, T ; L^{2}\right)}^{2} \leq\|\rho\|_{L^{2}\left(0, T ; L^{2}\right)}^{2},
$$

and the assertion of the lemma follows.

We use the energy identity (3.5) to conclude after twice integrating in time, for $T$ a point of the net $I_{k}:=\left\{t_{k}\right\}$,

$$
\frac{1}{2} \int_{0}^{T}\left\|\sigma^{+}\right\|_{h}^{2} \mathrm{~d} s+\int_{0}^{T} \int_{0}^{t}\left[\frac{k}{2}\left\|\sigma_{t}\right\|_{h}^{2}+\alpha h^{\alpha}\left\|\nabla \sigma^{+}\right\|^{2}\right] \mathrm{d} s \mathrm{~d} t=\frac{T}{2}\left\|\rho^{0}\right\|_{h}^{2} .
$$

We combine equations (3.21), (3.18) as well as the following bounds which employ (3.4), (3.5), and (2.4),

$$
\begin{aligned}
\int_{0}^{T}\left\|\sigma^{+}-\sigma\right\|_{h}^{2} \mathrm{~d} s & \leq C k\left(k \int_{0}^{T}\left\|\sigma_{t}\right\|_{h}^{2} \mathrm{~d} s\right) \rightarrow 0 \\
\int_{0}^{T}\left|\|\sigma\|^{2}-\|\sigma\|_{h}^{2}\right| \mathrm{d} s & \leq C h\|\sigma\|_{L^{2}\left(0, T ; L^{2}\right)}\|\sigma\|_{L^{2}\left(0, T ; W^{1,2}\right)} \leq C h^{1-\frac{\alpha}{2}} \rightarrow 0
\end{aligned}
$$

for $k, h \rightarrow 0$, in case $1-\frac{\alpha}{2}>0$, to obtain

$$
\begin{aligned}
& \frac{1}{2}\|\rho\|_{L^{2}\left(0, T ; L^{2}\right)}^{2}=\liminf _{k, h \rightarrow 0}\left(\frac{1}{2} \int_{0}^{T}\left\|\sigma^{+}\right\|_{h}^{2} \mathrm{~d} s\right. \\
&\left.+\int_{0}^{T} \int_{0}^{t}\left[\frac{k}{2}\left\|\sigma_{t}\right\|_{h}^{2}+\alpha h^{\alpha}\left\|\nabla \sigma^{+}\right\|^{2}\right] \mathrm{d} s \mathrm{~d} t\right) \\
& \geq \liminf _{k, h \rightarrow 0}\left(\frac{1}{2}\|\sigma\|_{L^{2}\left(0, T ; L^{2}\right)}^{2}+\int_{0}^{T} \int_{0}^{t}\left[\frac{k}{2}\left\|\sigma_{t}\right\|_{h}^{2}+\alpha h^{\alpha}\left\|\nabla \sigma^{+}\right\|^{2}\right] \mathrm{d} s \mathrm{~d} t\right) .
\end{aligned}
$$

This implies (3.19), and for almost every $t \in[0, T]$ we may conclude that

$$
\liminf _{k, h \rightarrow 0} \int_{0}^{t}\left[\frac{k}{2}\left\|\sigma_{t}\right\|^{2}+\alpha h^{\alpha}\left\|\nabla \sigma^{+}\right\|^{2}\right] \mathrm{d} s=0
$$

as well. 
In order to identify limits in each term of $(3.11)_{1-3}$, we need strong $L^{p}$-convergence of the sequence $\{(\boldsymbol{U}, \mathcal{B}, \sigma)\}$. For this purpose, we employ Lemma 2.4. because of the uniform bounds in Lemma 3.1, and Lemma 2.5] by [37] it remains to show that there exist a constant $C>0$ independent of $k, h>0$, and some $\kappa \in(0,1)$ such that

$$
\int_{\delta}^{T}\left[\|\mathcal{U}(t, \cdot)-\mathcal{U}(t-\delta, \cdot)\|_{\mathbf{L}^{2}}^{2}+\mathcal{B}(t, \cdot)-\mathcal{B}(t-\delta, \cdot) \|_{\mathbf{L}^{2}}^{2}\right] \mathrm{d} t \leq C \delta^{\kappa} \quad \forall \delta \in[0, T] .
$$

The proof of the following lemma requires $(\cdot, \cdot)_{*}=(\cdot, \cdot)_{h}$ in (3.2) of Scheme A, and $\beta_{3}>0$ for $d=3$.

Lemma 3.3. Let $(\cdot, \cdot)_{*}=(\cdot, \cdot)_{h}$ in (3.2), suppose (2.8), and choose $0<\alpha, \beta_{1}, \beta_{2}, \beta_{3}$ in Scheme A, such that

$$
0<\alpha+\frac{\beta_{2}}{2}<\frac{6-d}{6} \quad \text { and, moreover, for } d=3: \quad \alpha \geq \frac{\beta_{3}}{4} .
$$

There exists $\kappa \equiv \kappa(\Omega)>0$ such that iterates $\left\{\left(\mathbf{U}^{n}, \mathbf{B}^{n}\right)\right\}_{n=1}^{N} \subset \mathbf{J}_{h} \times \mathbf{X}_{h}$ of Scheme A satisfy

$$
\int_{\delta}^{T}\left[\|\boldsymbol{U}(t, \cdot)-\mathcal{U}(t-\delta, \cdot)\|_{\mathbf{L}^{2}}^{2}+\|\boldsymbol{B}(t, \cdot)-\mathcal{B}(t-\delta, \cdot)\|_{\mathbf{L}^{2}}^{2}\right] \mathrm{d} t \leq C \delta^{\kappa} \quad \forall \delta \in[0, T] .
$$

A corresponding result for the density dependent incompressible Navier-Stokes equation in a different numerical setting may be found in [30, pp. 1295-1296].

Proof. Step 1. Control of increments of $\left\{\mathbf{U}^{n}\right\} \subset \mathbf{J}_{h}$. Let $(\cdot, \cdot)_{*}=(\cdot, \cdot)_{h}$. Rewrite the leading two terms in equation (3.11) 2 as $\left([\sigma \mathcal{U}]_{t}, \mathbf{W}\right)_{h}-\frac{1}{2}\left(\sigma_{t} \mathcal{U}^{+}, \mathbf{W}\right)_{h}$. We use equation (3.11) 1 with $\chi=\mathcal{I}_{V_{h}}\left\langle\mathcal{U}^{+}, \mathbf{W}\right\rangle$ to conclude $\left(\mathbf{W} \in \mathbf{J}_{h}\right)$ :

$$
\begin{aligned}
\left(\sigma_{t} \mathcal{U}^{+}, \mathbf{W}\right)_{h}=( & \left.\sigma^{+} \mathcal{U}^{+}, \nabla \mathcal{I}_{V_{h}}\left\langle\mathcal{U}^{+}, \mathbf{W}\right\rangle\right)+\frac{1}{2}\left(\left[\operatorname{div} \mathcal{U}^{+}\right] \sigma^{+}, \mathcal{I}_{V_{h}}\left\langle\mathcal{U}^{+}, \mathbf{W}\right\rangle\right) \\
& -\alpha h^{\alpha}\left(\nabla \sigma^{+}, \nabla \mathcal{I}_{V_{h}}\left\langle\mathcal{U}^{+}, \mathbf{W}\right\rangle\right) .
\end{aligned}
$$

Let $t=t_{n} \geq t_{\ell} \geq 0$ be a point of the net $I_{k}=\left\{t_{n}\right\}_{n \geq 0}$. Integration with respect to $s \in\left(t_{n-\ell}, t_{n}\right)$, and using (

$$
\begin{aligned}
\left([\sigma \mathcal{U}]\left(t_{n}, \cdot\right)-[\sigma \mathcal{U}]\left(t_{n-\ell}, \cdot\right), \mathbf{W}\right)_{h}+\beta_{1} h^{-\beta_{1}} \int_{t_{n-\ell}}^{t_{n}}\left(\operatorname{div} \mathcal{U}^{+}, \operatorname{div} \mathbf{W}\right) \mathrm{d} s \\
\quad+\beta_{2} h^{\beta_{2}}\left(\nabla\left[\mathcal{U}\left(t_{n}, \cdot\right)-\mathcal{U}\left(t_{n-\ell}, \cdot\right)\right], \nabla \mathbf{W}\right)+\beta_{3} h^{\beta_{3}} \int_{t_{n-\ell}}^{t_{n}}\left(\Delta_{h} \mathcal{U}^{+}, \Delta_{h} \mathbf{W}\right) \mathrm{d} s \\
=\frac{1}{2} \int_{t_{n-\ell}}^{t_{n}}\left[\left(\sigma^{+} \mathcal{U}^{+}, \nabla \mathcal{I}_{V_{h}}\left\langle\mathcal{U}^{+}, \mathbf{W}\right\rangle\right)+\frac{1}{2}\left(\left[\operatorname{div} \mathcal{U}^{+}\right] \sigma^{+}, \mathcal{I}_{V_{h}}\left\langle\mathcal{U}^{+}, \mathbf{W}\right\rangle\right)\right. \\
\left.\quad-\alpha h^{\alpha}\left(\nabla \sigma^{+}, \nabla \mathcal{I}_{V_{h}}\left\langle\mathcal{U}^{+}, \mathbf{W}\right\rangle\right)\right] \mathrm{d} s \\
\quad-\int_{t_{n-\ell}}^{t_{n}}\left[\frac{1}{\bar{\mu}}\left(\boldsymbol{B}^{-} \times \operatorname{curl} \mathcal{B}^{+}, \mathbf{W}\right)-\left(\sigma^{-} \mathbf{g}^{+}, \mathbf{W}\right)\right] \mathrm{d} s \\
\quad-\int_{t_{n-\ell}}^{t_{n}} \bar{A} \mathrm{~d} s=: I+. .+V-\int_{t_{n-\ell}}^{t_{n}} \bar{A} \mathrm{~d} s,
\end{aligned}
$$

where $\bar{A}$ summarizes the terms three to five in $(3.11)_{2}$. 
Correspondingly, integrating (3.11) 1 with respect to $s \in\left(t_{n-\ell}, t_{n}\right)$, and setting $\chi=\mathcal{I}_{V_{h}}\left\langle\mathcal{U}\left(t_{n}, \cdot\right), \mathbf{W}\right\rangle$ leads to

$$
\begin{aligned}
& \left(\sigma\left(t_{n}, \cdot\right)-\sigma\left(t_{n-\ell}, \cdot\right),\left\langle\mathcal{U}\left(t_{n}, \cdot\right), \mathbf{W}\right\rangle\right)_{h}=\int_{t_{n-\ell}}^{t_{n}}\left[\left(\sigma^{+} \mathcal{U}^{+}, \nabla \mathcal{I}_{V_{h}}\left\langle\mathcal{U}\left(t_{n}, \cdot\right), \mathbf{W}\right\rangle\right)\right. \\
& \quad+\frac{1}{2}\left(\left[\operatorname{div} \mathcal{U}^{+}\right] \sigma^{+}, \mathcal{I}_{V_{h}}\left\langle\mathcal{U}\left(t_{n}, \cdot\right), \mathbf{W}\right\rangle\right) \\
& \left.\quad-\alpha h^{\alpha}\left(\nabla \sigma^{+}, \nabla \mathcal{I}_{V_{h}}\left\langle\mathcal{U}\left(t_{n}, \cdot\right), \mathbf{W}\right\rangle\right)\right] \mathrm{d} s=: V I+. .+X
\end{aligned}
$$

Subtracting this equation from (3.24) and observing that

$$
\begin{aligned}
& \left([\sigma \mathcal{U}]\left(t_{n}, \cdot\right)-[\sigma \mathcal{U}]\left(t_{n-\ell}, \cdot\right), \mathbf{W}\right)_{h}-\left(\sigma\left(t_{n}, \cdot\right)-\sigma\left(t_{n-\ell}, \cdot\right),\left\langle\mathcal{U}\left(t_{n}, \cdot\right), \mathbf{W}\right\rangle\right)_{h} \\
& \quad=\left(\sigma\left(t_{n-\ell}, \cdot\right)\left[\mathcal{U}\left(t_{n}, \cdot\right)-\mathcal{U}\left(t_{n-\ell}, \cdot\right)\right], \mathbf{W}\right)_{h}
\end{aligned}
$$

leads to

$$
\begin{gathered}
\left(\sigma\left(t_{n-\ell}\right)\left[\mathcal{U}\left(t_{n}, \cdot\right)-\mathcal{U}\left(t_{n-\ell}, \cdot\right)\right], \mathbf{W}\right)_{h}+\beta_{1} h^{-\beta_{1}} \int_{t_{n-\ell}}^{t_{n}}\left(\operatorname{div} \mathcal{U}^{+}, \operatorname{div} \mathbf{W}\right) \\
+\beta_{2} h^{\beta_{2}}\left(\nabla\left[\mathcal{U}\left(t_{n}, \cdot\right)-\mathcal{U}\left(t_{n-\ell}, \cdot\right)\right], \nabla \mathbf{W}\right)+\beta_{3} h^{\beta_{3}} \int_{t_{n-\ell}}^{t_{n}}\left(\Delta_{h} \mathcal{U}^{+}, \Delta_{h} \mathbf{W}\right) \mathrm{d} s \\
=I+. .+V-\int_{t_{n-\ell}}^{t_{n}} \bar{A} \mathrm{~d} s-\int_{t_{n-\ell}}^{t_{n}}[V I+. .+X] \mathrm{d} s
\end{gathered}
$$

Upon choosing $\mathbf{W}=\mathcal{U}\left(t_{n}, \cdot\right)-\mathcal{U}\left(t_{n-\ell}, \cdot\right) \in \mathbf{J}_{h}$, and summing over all steps $\ell \leq n \leq$ $N$, and using (3.6), the left-hand side dominates

$$
\begin{gathered}
k \sum_{n=\ell}^{N}\left[\bar{\rho}_{1}\left\|\boldsymbol{U}\left(t_{n}, \cdot\right)-\mathcal{U}\left(t_{n-\ell}, \cdot\right)\right\|_{h}^{2}+\beta_{2} h^{\beta_{2}}\left\|\nabla\left[\boldsymbol{U}\left(t_{n}, \cdot\right)-\mathcal{U}\left(t_{n-\ell}, \cdot\right)\right]\right\|^{2}\right] \\
\quad-\frac{\beta_{1}}{h^{\beta_{1}}} k \sum_{n=\ell}^{N}\left|\int_{t_{n-\ell}}^{t_{n}}\left(\operatorname{div} \mathcal{U}^{+}, \operatorname{div}\left[\mathcal{U}\left(t_{n}, \cdot\right)-\mathcal{U}\left(t_{n-\ell}, \cdot\right)\right]\right) \mathrm{d} s\right| \\
\quad-\beta_{3} h^{\beta_{3}} k \sum_{n=\ell}^{N}\left|\int_{t_{n-\ell}}^{t_{n}}\left(\Delta_{h} \mathcal{U}^{+}, \Delta_{h}\left[\boldsymbol{U}\left(t_{n}, \cdot\right)-\mathcal{U}\left(t_{n-\ell}, \cdot\right)\right]\right) \mathrm{d} s\right|
\end{gathered}
$$

Next, we derive bounds $C t_{\ell}^{\kappa}$, for some $\kappa>0$ for each term on the right-hand side of (3.26), after integration in time over $[0, T]$.

By (3.4), the negative term in (3.27) may then be bounded by

$$
\begin{aligned}
\leq & \frac{\beta_{1}}{h^{\beta_{1}}} k \sum_{n=\ell}^{N}\left\{\left\|\operatorname{div} \mathcal{U}\left(t_{n}, \cdot\right)\right\|+\left\|\operatorname{div} \mathcal{U}\left(t_{n-\ell}, \cdot\right)\right\|\right\} \int_{t_{n-\ell}}^{t_{n}}\left\|\operatorname{div} \mathcal{U}^{+}\right\| \mathrm{d} s \\
& +\beta_{3} h^{\beta_{3}} k \sum_{n=\ell}^{N}\left\{\left\|\Delta_{h} \mathcal{U}\left(t_{n}, \cdot\right)\right\|+\left\|\Delta_{h} \mathcal{U}\left(t_{n-\ell}, \cdot\right)\right\|\right\} \int_{t_{n-\ell}}^{t_{n}}\left\|\Delta_{h} \mathcal{U}^{+}\right\| \mathrm{d} s \leq C t_{\ell} .
\end{aligned}
$$


Next, we bound $k \sum_{n=\ell}^{N}(I+I I)$. For this purpose, interpolate $L^{3}$ between $L^{2}$ and $W^{1,2}$, and use $W^{1,2} \hookrightarrow L^{6}$ to conclude

$$
\begin{aligned}
I+I I & \left.\leq C \int_{t_{n-\ell}}^{t_{n}}\left\|\sigma^{+}\right\|_{L^{\infty}}\left\|\mathcal{U}^{+}\right\|_{\mathbf{L}^{3}}\left\|\nabla \mathcal{U}^{+}\right\| \| \nabla \boldsymbol{U}\left(t_{n}, \cdot\right)-\mathcal{U}\left(t_{n-\ell}, \cdot\right)\right] \| \mathrm{d} s \\
& \leq C \sup _{[0, T]}\left\|\mathcal{U}^{+}\right\|^{1 / 2} k \sum_{n=\ell}^{N}\left[\left\|\nabla \boldsymbol{U}\left(t_{n}, \cdot\right)\right\|+\left\|\nabla \boldsymbol{U}\left(t_{n-\ell}, \cdot\right)\right\|\right] \int_{t_{n-\ell}}^{t_{n}}\left\|\nabla \mathcal{U}^{+}\right\|^{3 / 2} \mathrm{~d} s \\
& \leq C k \sum_{n=0}^{N}\left\|\nabla \mathcal{U}^{+}\right\|\left(\int_{t_{n-\ell}}^{t_{n}}\left\|\nabla \mathcal{U}^{+}\right\|^{2} \mathrm{~d} s\right)^{3 / 4} t_{\ell}^{1 / 4} \leq C t_{\ell}^{1 / 4} .
\end{aligned}
$$

A corresponding estimate for $k \sum_{n=1}^{N} \int_{t_{n-\ell}}^{t_{n}} \bar{A} \mathrm{~d} s$ follows accordingly.

The term $I I I$ in (3.26) requires $\beta_{3}>0$ for $d=3$ in order not to conflict with the requirement for $0<\alpha, \beta_{2}$ needed for property (3.6); see Lemma 3.1. We confine to the case $d=3$; summing over $\ell \leq n \leq N$ leads to

$$
\begin{aligned}
& \alpha h^{\alpha} k \sum_{n=\ell}^{N} \int_{t_{n-\ell}}^{t_{n}}\left|\left(\nabla \sigma^{+}, \nabla \mathcal{I}_{V_{h}}\left\langle\mathcal{U}^{+}, \mathbf{W}\right\rangle\right)\right| \mathrm{d} s \\
& \leq C \alpha h^{\alpha} k \sum_{n=\ell}^{N} \int_{t_{n-\ell}}^{t_{n}}\left\|\nabla \sigma^{+}\right\|_{\mathbf{L}^{2}}\left[\left\|\nabla \boldsymbol{U}^{+}\right\|_{\mathbf{L}^{2}}\left\|\boldsymbol{U}\left(t_{n}, \cdot\right)-\mathcal{U}\left(t_{n-\ell}, \cdot\right)\right\|_{\mathbf{L}^{\infty}}\right. \\
& \left.\quad+\left\|\mathcal{U}^{+}\right\|_{\mathbf{L}^{\infty}}\left\|\nabla\left[\mathcal{U}\left(t_{n}, \cdot\right)-\boldsymbol{U}\left(t_{n-\ell}, \cdot\right)\right]\right\|_{\mathbf{L}^{2}}\right] \mathrm{d} s \\
& \leq C \sqrt{\alpha} h^{\alpha / 2} t_{\ell}^{1 / 2} \max _{\ell \leq n \leq N}\left(\alpha h^{\alpha} \int_{t_{n-\ell}}^{t_{n}}\left\|\nabla \sigma^{+}\right\|^{2} \mathrm{~d} s\right)^{1 / 2} \\
& \quad \times k \sum_{n=\ell}^{N}\left\|\nabla \mathbf{U}^{n}\right\|_{\mathbf{L}^{2}}^{5 / 4}\left(\left\|\mathbf{U}^{n}\right\|+\left\|\Delta_{h} \mathbf{U}^{n}\right\|\right)^{3 / 4} \\
& \leq C \sqrt{\alpha} t_{\ell}^{1 / 2} h^{\frac{\alpha}{2}-\frac{3 \beta_{3}}{8}}\left(k \sum_{n=0}^{N}\left\|\nabla \mathbf{U}^{n}\right\|^{2}\right)^{5 / 8}\left(h^{\beta_{3}} k \sum_{n=0}^{N}\left\|\Delta_{h} \mathbf{U}^{n}\right\|^{2}\right)^{3 / 8} .
\end{aligned}
$$

Here, we use (3.4), (2.10), Hölder, and an inverse inequality. As a consequence, we need

$$
\frac{\alpha}{2} \geq \frac{3 \beta_{3}}{8} \quad(d=3)
$$

In order to control $I V$, we use property (2.15) of the Hodge mapping $\mathbf{H}: \mathbf{X}_{h} \rightarrow \mathbf{X}$, for some $\sigma \equiv \sigma(\Omega)>0$. We compute $\left(\mathbf{W}=\mathcal{U}\left(t_{n}, \cdot\right)-\mathcal{U}\left(t_{n-\ell}, \cdot\right)\right)$

$$
\begin{array}{r}
\left|\left(\mathcal{B}^{-} \times \operatorname{curl} \mathcal{B}^{+}, \mathbf{W}\right)\right| \leq\left\|\mathcal{B}^{-}-\mathbf{H}\left(\mathcal{B}^{-}\right)\right\|_{\mathbf{L}^{2}}\left\|\operatorname{curl} \mathcal{B}^{+}\right\|_{\mathbf{L}^{2}}\|\mathbf{W}\|_{\mathbf{L}^{\infty}} \\
+\left\|\mathbf{H}\left(\mathcal{B}^{-}\right)\right\|_{\mathbf{L}^{3}}\left\|\operatorname{curl} \mathcal{B}^{+}\right\|_{\mathbf{L}^{2}}\|\mathbf{W}\|_{\mathbf{L}^{6}}
\end{array}
$$

We use an inverse estimate for the first term, (2.15), and $L^{p}$-interpolation together with Lemma 2.1 (ii) for the second. Then, there exists $0<\delta_{2} \equiv \delta_{2}\left(\delta_{1}\right)<1$, such 
that we find the upper bound

$$
\begin{aligned}
& \leq C\left[h^{\sigma}\left\|\operatorname{curl} \mathcal{B}^{-}\right\|_{\mathbf{L}^{2}}\left\|\operatorname{curl} \mathcal{B}^{+}\right\|_{\mathbf{L}^{2}}\right. \\
& \left.\quad+\left\|\mathcal{B}^{-}\right\|_{\mathbf{L}^{2}}^{\delta_{2}}\left\|\mathcal{B}^{-}\right\|_{\mathbf{H}(\operatorname{curl})}^{1-\delta_{2}}\left\|\operatorname{curl} \mathcal{B}^{+}\right\|_{\mathbf{L}^{2}}\right]\|\mathbf{W}\|_{\mathbf{L}^{6}} \\
& \leq C\left[\left\|\mathcal{B}^{-}\right\|_{\mathbf{L}^{2}}+\left\|\mathcal{B}^{-}\right\|_{\mathbf{L}^{2}}^{\delta_{2}}\left\|\operatorname{curl} \mathcal{B}^{-}\right\|_{\mathbf{L}^{2}}^{1-\delta_{2}}\right]\left\|\operatorname{curl} \mathcal{B}^{+}\right\|_{\mathbf{L}^{2}}\|\nabla \mathbf{W}\|_{\mathbf{L}^{2}} .
\end{aligned}
$$

Thanks to the discrete energy law (3.4), we may then conclude as follows:

$$
\begin{aligned}
& k \sum_{n=\ell}^{N} \int_{t_{n-\ell}}^{t_{n}}\left|\left(\mathcal{B}^{-} \times \operatorname{curl} \mathcal{B}^{+}, \mathbf{W}\right)\right| \mathrm{d} s \\
& \quad \leq C k \sum_{n=\ell}^{N} \int_{t_{n-\ell}}^{t_{n}}\left[\left\|\operatorname{curl} \mathcal{B}^{-}\right\|+\left\|\operatorname{curl} \mathcal{B}^{+}\right\|\right]^{2-\delta_{2}}\|\nabla \mathbf{W}\| \mathrm{d} s \\
& \quad \leq C\left(k \sum_{n=\ell}^{N}\|\nabla \mathbf{W}\|\right) t_{\ell}^{\frac{\delta_{2}}{2}} \max _{\ell \leq n \leq N}\left(\int_{t_{n-\ell}}^{t_{n}}\left[\left\|\operatorname{curl} \mathcal{B}^{-}\right\|+\left\|\operatorname{curl} \mathcal{B}^{+}\right\|\right]^{2} \mathrm{~d} s\right)^{\frac{2-\delta_{2}}{2}} \\
& \quad \leq C t_{\ell}^{\frac{\delta_{2}}{2}} .
\end{aligned}
$$

Putting things together then yields for some $\kappa \equiv \kappa(\Omega)>0$,

$$
\int_{\delta}^{T}\|\mathcal{U}(t, \cdot)-\mathcal{U}(t-\delta, \cdot)\|^{2} \mathrm{~d} t \leq C \delta^{\kappa} \quad \forall \delta \in[0, T] .
$$

Step 2. Control of increments of $\left\{\mathbf{B}^{n}\right\} \subset \mathbf{X}_{h}$. We proceed similarly to Step 1, where arguments simplify and we use equation (3.11) 3 .

Let $t=t_{n}>0$ be a point of the net $I_{k}$. Integrating (3.11) 3 with respect to $s \in\left(t_{n-\ell}, t_{n}\right)$ leads to

$$
\left(\mathcal{B}\left(t_{n}, \cdot\right)-\mathcal{B}\left(t_{n-\ell}, \cdot\right), \boldsymbol{\psi}\right)=-\int_{t_{n-\ell}}^{t_{n}}\left[\frac{1}{\bar{\mu}}\left(\frac{1}{\xi^{-}} \operatorname{curl} \mathcal{B}^{+}, \operatorname{curl} \psi\right)+\left(\mathcal{U}^{+} \times \mathcal{B}^{-}, \operatorname{curl} \psi\right)\right] \mathrm{d} s .
$$

Upon choosing $\boldsymbol{\psi}=\mathcal{B}\left(t_{n}, \cdot\right)-\mathcal{B}\left(t_{n-\ell}, \cdot\right) \in \mathbf{X}_{h}$, the left-hand side dominates $\| \mathcal{B}\left(t_{n}, \cdot\right)-$ $\mathcal{B}\left(t_{n-\ell}, \cdot\right) \|^{2}$; after summation over $\ell \leq n \leq N$, terms on the right-hand side may be bounded as

$$
\frac{1}{\bar{\xi}_{-}} k \sum_{n=\ell}^{N} \int_{t_{n-\ell}}^{t_{n}}\left(\operatorname{curl} \mathcal{B}^{+}, \operatorname{curl} \psi\right) \mathrm{d} s \leq C t_{\ell} k \sum_{n=\ell}^{N}\left[\left\|\operatorname{curl} \mathcal{B}^{-}\right\|+\left\|\operatorname{curl} \mathcal{B}^{+}\right\|\right]^{2} \leq C t_{\ell},
$$

thanks to (3.4). We use similar arguments as in the previous step to control the term $I V$ there.

We use the inverse estimate $\left\|\boldsymbol{U}^{+}\right\|_{\mathbf{L}^{\infty}} \leq C h^{-1 / 2}\left\|\boldsymbol{U}^{+}\right\|_{\mathbf{L}^{2}}$, estimate (2.15), and Lemma 2.1 (ii) to conclude that

$$
\begin{aligned}
& \left|\left(\mathcal{U}^{+} \times \mathcal{B}^{-}, \operatorname{curl} \psi\right)\right| \leq\left\|\mathcal{U}^{+}\right\|_{\mathbf{L}^{\infty}}\left\|\mathcal{B}^{-}-\mathbf{H}\left(\mathcal{B}^{-}\right)\right\|_{\mathbf{L}^{2}}\|\operatorname{curl} \psi\|_{\mathbf{L}^{2}} \\
& \quad+\left\|\mathcal{U}^{+}\right\|_{\mathbf{L}^{6}}\left\|\mathbf{H}\left(\mathcal{B}^{-}\right)\right\|_{\mathbf{L}^{3}}\|\operatorname{curl} \psi\|_{\mathbf{L}^{2}} \\
& \leq C\left[h^{\tilde{\sigma}}\left\|\operatorname{curl} \mathcal{B}^{-}\right\|_{\mathbf{L}^{2}}+\left\|\mathcal{B}^{-}\right\|^{\delta_{2}}\left\|\mathcal{B}^{-}\right\|_{\mathbf{H}(\operatorname{curl})}^{1-\delta_{2}}\right]\left\|\mathcal{U}^{+}\right\|_{\mathbf{L}^{6}}\|\operatorname{curl} \psi\|_{\mathbf{L}^{2}}
\end{aligned}
$$


for some $\tilde{\sigma} \equiv \widetilde{\sigma}(\Omega)>0$, and $0<\delta_{2} \equiv \delta_{2}\left(\delta_{1}\right)<1$. Hence, there exists $\delta_{3} \equiv$ $\delta_{3}\left(\sigma, \delta_{2}\right)>0$, such that

$$
\begin{aligned}
& \int_{0}^{T} \int_{t_{n-\ell}}^{t_{n}}\left|\left(\mathcal{U}^{+} \times \mathcal{B}^{-}, \operatorname{curl} \boldsymbol{\psi}\right)\right| \mathrm{d} s \\
& \quad \leq C k \sum_{n=\ell}^{N} \int_{t_{n-\ell}}^{t_{n}}\left[1+\left\|\operatorname{curl} \mathcal{B}^{-}\right\|+\left\|\operatorname{curl} \mathcal{B}^{+}\right\|\right]^{2-\delta_{3}}\left\|\nabla \mathcal{U}^{+}\right\| \mathrm{d} s \\
& \quad \leq C t_{\ell}^{\frac{\delta_{3}}{2}} k \sum_{n=\ell}^{N}\left\|\nabla \mathcal{U}^{+}\right\| \max _{\ell \leq n \leq N}\left(\int_{t_{n-\ell}}^{t_{n}}\left[\left\|\operatorname{curl} \mathcal{B}^{-}\right\|+\left\|\operatorname{curl} \mathcal{B}^{+}\right\|\right]^{2} \mathrm{~d} s\right)^{\frac{2-\delta_{3}}{2}} \leq C t_{\ell}^{\frac{\delta_{3}}{2}}
\end{aligned}
$$

Putting things together yields for some existing $\kappa \equiv \kappa(\Omega)>0$ that

$$
\int_{\delta}^{T}\|\mathcal{B}(t, \cdot)-\mathcal{B}(t-\delta, \cdot)\|^{2} \mathrm{~d} s \leq C \delta^{\kappa} \quad \forall \delta \in[0, T] .
$$

By Lemma 2.4 we may conclude from Lemma 3.3 compactness of the sequence $\{(\boldsymbol{U}, \mathcal{B})\} \subset\left[L^{2}\left(0, T ; L^{2}\left(\Omega, \mathbb{R}^{3}\right)\right)\right]^{2}$ if $\alpha, \beta_{1}, \beta_{2}, \beta_{3}>0$, and hence in addition to (3.13) for $k, h \rightarrow 0$,

$$
\begin{aligned}
\mathcal{U}^{ \pm}, \mathcal{U} \rightarrow \mathbf{u} & \text { in } L^{2}\left(0, T ; \mathbf{L}^{2}\right), \\
\mathcal{B}^{ \pm}, \mathcal{B} \rightarrow \mathbf{b} & \text { in } L^{2}\left(0, T ; \mathbf{L}^{2}\right), \\
\sigma^{ \pm}, \sigma \rightarrow \rho & \text { in } L^{q}\left(0, T ; L^{q}\right) \quad(1 \leq q<\infty),
\end{aligned}
$$

where property (3.31) 3 is a consequence of the uniform bounds for $\{\sigma\} \subset$ $L^{\infty}\left(0, T ; L^{\infty}\right)$, and Lemma 3.2. together with (3.13) 6 .

It remains to pass to limits in each of the terms in (3.11) 2 and $(3.11)_{3}$ for $k, h \rightarrow 0$, with $(\cdot, \cdot)_{*}=(\cdot, \cdot)_{h}$. We begin with (3.11) 2 , where $\mathbf{W}(t, \cdot)=\mathbf{Q}_{h}^{1} \boldsymbol{\zeta}(t, \cdot)$ for every $t \in[0, T)$, and $\zeta \in C_{0}^{\infty}([0, T) ; \mathcal{J})$. We rewrite the leading two terms in $\left({ }_{3.11}\right)_{2}$ as follows:

$$
\begin{aligned}
\frac{1}{2}\left\{\left(\sigma^{-} \mathcal{U}_{t}, \mathbf{W}\right)_{h}+\left([\sigma \mathcal{U}]_{t}, \mathbf{W}\right)_{h}\right\}= & \left([\sigma \mathcal{U}]_{t}, \mathbf{W}\right)+\left[\left([\sigma \mathcal{U}]_{t}, \mathbf{W}\right)_{h}-\left([\sigma \mathcal{U}]_{t}, \mathbf{W}\right)\right] \\
& -\frac{1}{2}\left(\sigma_{t} \mathcal{U}^{+}, \mathbf{W}\right)_{h} .
\end{aligned}
$$

We independently control terms on the right-hand side:

(i) Last term on thee right-hand side of (3.32). We use (3.23), which has three terms $A_{1}, A_{2}$, and $A_{3}$ on the right-hand side:

$$
A_{1}=\left(\left[\sigma^{+} \mathcal{U}^{+}\right], \nabla\left\langle\mathcal{U}^{+}, \mathbf{W}\right\rangle\right)+\left(\operatorname{div}\left[\sigma^{+} \mathcal{U}^{+}\right],\left[\mathcal{I}_{V_{h}}-\mathrm{Id}\right]\left\langle\mathcal{U}^{+}, \mathbf{W}\right\rangle\right)=: A_{11}+A_{12} .
$$

We easily conclude $\lim _{k, h \rightarrow 0} A_{11}=(\rho \mathbf{u}, \nabla\langle\mathbf{u}, \zeta\rangle)$ from (3.13), (3.31) 3,1 . We use an inverse estimate, and a standard approximation result to bound the term $A_{12}$,

$$
\begin{aligned}
\left|A_{12}\right| & \leq\left\|\operatorname{div}\left[\sigma^{+} \boldsymbol{U}^{+}\right]\right\|\left\|\left[\mathcal{I}_{V_{h}}-\mathrm{Id}\right]\left\langle\mathcal{U}^{+}, \mathbf{W}\right\rangle\right\| \\
& \leq\left(\left\|\sigma^{+}\right\|_{L^{\infty}}\left\|\operatorname{div} \mathcal{U}^{+}\right\|+\left\|\nabla \sigma^{+}\right\|\left\|\mathcal{U}^{+}\right\|_{\mathbf{L}^{\infty}}\right) C h\left\|\nabla\left\langle\boldsymbol{U}^{+}, \mathbf{W}\right\rangle\right\| \\
& \leq\left(h^{\frac{\alpha}{2}}\left\|\nabla \sigma^{+}\right\|\right) C h^{1-\ell_{d}-\frac{\alpha}{2}-\frac{\beta_{2}}{2}}\left(h^{\frac{\beta_{2}}{2}}\left\|\nabla \mathcal{U}^{+}\right\|\right)\left\|\nabla\left\langle\mathcal{U}^{+}, \mathbf{W}\right\rangle\right\|,
\end{aligned}
$$

for any $\ell_{2}>0$, and $\ell_{3}=\frac{1}{2}$. Thanks to (3.4), (3.5), in order to validate $\int_{0}^{T}\left|A_{12}\right| \mathrm{d} s \rightarrow$ 0 for $k, h \rightarrow 0$, we have to make sure that $1-\ell_{d}-\frac{\alpha}{2}-\frac{\beta_{2}}{2}>0$, which is valid in case we have $\alpha+\beta_{2}<\frac{6-d}{d}$, and $\sqrt{\beta_{2}} h^{\frac{\beta_{2}}{2}}\left\|\nabla \mathbf{U}^{0}\right\| \leq C$. 
Identifying $\lim _{k, h \rightarrow 0}\left[\left|A_{2}\right|+\left|A_{3}\right|\right]=0$ in (3.23) follows easily from (3.13), (3.31), and (3.5).

(ii) Difference term on right-hand side of (3.32). We use integration by parts to conclude that

$$
\begin{aligned}
\mid \int_{0}^{T}([ & \left.\sigma \mathcal{U}]_{t}, \mathbf{W}\right)_{h}-\left([\sigma \mathcal{U}]_{t}, \mathbf{W}\right) \mathrm{d} s \mid \\
\leq & \int_{0}^{T}\left|\left(\sigma \mathcal{U}, \mathbf{W}_{t}\right)_{h}-\left(\sigma \mathcal{U}, \mathbf{W}_{t}\right)\right| \mathrm{d} s \\
& +\left|\left(\rho^{0} \mathbf{U}^{0}, \mathbf{W}(0, \cdot)\right)_{h}-\left(\rho^{0} \mathbf{U}^{0}, \mathbf{W}(0, \cdot)\right)\right| \\
\leq & C h \int_{0}^{T}\|\sigma\|_{L^{\infty}}\|\mathbf{U}\|_{\mathbf{L}^{2}}\left\|\boldsymbol{\zeta}_{t}\right\|_{\mathbf{W}^{1,2}} \mathrm{~d} s \\
& +C h\left\|\rho_{0}\right\|_{L^{\infty}}\left\|\mathbf{u}_{0}\right\|\|\boldsymbol{\zeta}\|_{\mathbf{W}^{1,2}} \rightarrow 0 \quad(k, h \rightarrow 0) .
\end{aligned}
$$

(iii) First term on right-hand side of (3.32). We use integration by parts to conclude

$$
\begin{aligned}
\int_{0}^{T}\left([\sigma \mathcal{U}]_{t}, \mathbf{W}\right) \mathrm{d} s & =-\int_{0}^{T}\left(\sigma \mathcal{U}, \mathbf{W}_{t}\right) \mathrm{d} s-\left(\rho^{0} \mathbf{U}^{0}, \mathbf{W}(0, \cdot)\right) \\
& \rightarrow-\int_{0}^{T}\left(\rho \mathbf{u}, \boldsymbol{\zeta}_{t}\right) \mathrm{d} s-\left(\rho_{0} \mathbf{u}_{0}, \boldsymbol{\zeta}(0, \cdot)\right) \quad(k, h \rightarrow 0)
\end{aligned}
$$

Putting (i) through (iii) together yields for (3.32),

$$
\begin{aligned}
\frac{1}{2} \lim _{k, h \rightarrow 0} & \int_{0}^{T}\left[\left(\sigma^{-} \mathcal{U}_{t}, \mathbf{W}\right)_{h}+\left([\sigma \mathcal{U}]_{t}, \mathbf{W}\right)_{h}\right] \mathrm{d} s \\
= & \int_{0}^{T}\left[\frac{1}{2}(\rho \mathbf{u}, \nabla\langle\mathbf{u}, \boldsymbol{\zeta}\rangle)-\left(\rho \mathbf{u}, \boldsymbol{\zeta}_{t}\right)\right] \mathrm{d} s-\left(\rho_{0} \mathbf{u}_{0}, \boldsymbol{\zeta}(0, \cdot)\right) \\
= & \frac{1}{2} \int_{0}^{T}[([\rho \mathbf{u} \cdot \nabla] \mathbf{u}, \boldsymbol{\zeta})+([\rho \mathbf{u} \cdot \nabla] \boldsymbol{\zeta}, \mathbf{u})] \mathrm{d} s \\
& -\int_{0}^{T}\left(\rho \mathbf{u}, \boldsymbol{\zeta}_{t}\right) \mathrm{d} s-\left(\rho_{0} \mathbf{u}_{0}, \boldsymbol{\zeta}(0, \cdot)\right) .
\end{aligned}
$$

Passing to the limit in the third and fourth term in $(3.11)_{2}$ uses strong $L^{p}$-convergence of density and velocity iterates given in $(3.31)_{1,3}$. For example, we find in a first step for the third term, using $(3.31)_{3}$, interpolation of $\mathbf{L}^{3}$ between $\mathbf{L}^{2}$ and $\mathbf{W}^{1,2}$, and Lemma 3.1 ,

$$
\begin{aligned}
\int_{0}^{T}\left|\left(\left[\left\{\sigma^{-}-\rho\right\} \mathcal{U}^{-} \cdot \nabla\right] \mathcal{U}^{+}, \mathbf{W}\right)\right| \mathrm{d} s \\
\leq\left(\int_{0}^{T}\left\|\sigma^{-}-\rho\right\|_{L^{6}}^{6} \mathrm{~d} s\right)^{\frac{1}{6}}\left(\int_{0}^{T}\left\|\mathcal{U}^{-}\right\|_{\mathbf{L}^{3}}^{3} \mathrm{~d} s\right)^{\frac{1}{3}} \\
\quad \times\left(\int_{0}^{T}\left\|\nabla \mathcal{U}^{+}\right\|_{\mathbf{L}^{2}}^{2} \mathrm{~d} s\right)^{\frac{1}{2}}\|\mathbf{W}\|_{L^{\infty}\left(0, T ; \mathbf{L}^{\infty}\right)} \rightarrow 0,
\end{aligned}
$$


for $k, h \rightarrow 0$; in a second step, we employ (3.31) 1 to conclude that

$$
\begin{aligned}
\int_{0}^{T} \mid & \left(\left[\rho\left\{\mathbf{u}-\mathcal{U}^{-}\right\} \cdot \nabla\right] \mathcal{U}^{+}, \mathbf{W}\right) \mid \mathrm{d} s \\
\leq\|\rho\|_{L^{\infty}\left(0, T ; L^{\infty}\right)} & \left(\int_{0}^{T}\left\|\mathbf{u}-\mathcal{U}^{-}\right\|_{\mathbf{L}^{2}}^{2} \mathrm{~d} s\right)^{1 / 2} \\
& \times\left(\int_{0}^{T}\left\|\nabla \mathcal{U}^{+}\right\|_{\mathbf{L}^{2}}^{2} \mathrm{~d} s\right)^{1 / 2}\|\mathbf{W}\|_{L^{\infty}\left(0, T ; \mathbf{L}^{\infty}\right)} \rightarrow 0
\end{aligned}
$$

for $k, h \rightarrow 0$. As a consequence, we find for the third term for $k, h \rightarrow 0$,

$$
\lim _{k, h \rightarrow 0} \int_{0}^{T}\left(\left[\sigma^{-} \mathcal{U}^{-} \cdot \nabla\right] \mathcal{U}^{+}, \mathbf{W}\right) \mathrm{d} s \rightarrow \int_{0}^{T}([\rho \mathbf{u} \cdot \nabla] \mathbf{u}, \zeta) \mathrm{d} s
$$

A convergence study for the fourth term in $\left({ }^{3.11}\right)_{2}$ is similar. Passing to the limit in terms $V, V I I-I X$ in equation (3.11) 2 follows easily from (3.13), (3.31). We recall the cancellation of terms from (3.33), and limits of $I I I, I V$.

For the sixth term in (3.11) 2 , we employ (3.4), (2.6) to conclude for $2>\beta_{1}$,

$$
\beta_{1} h^{-\beta_{1}}\left|\int_{0}^{T}\left(\operatorname{div} \mathcal{U}^{+}, \operatorname{div}[\mathbf{W}-\boldsymbol{\zeta}]\right) \mathrm{d} s\right| \leq C \beta_{1} h^{-\frac{\beta_{1}}{2}} h\|\boldsymbol{\zeta}\|_{L^{2}\left(0, T ; \mathbf{H}^{2}\right)} \rightarrow 0 \quad(k, h \rightarrow 0) .
$$

The limit of the seventh and eighth term in (3.11) 2 is zero for $\beta_{2}, \beta_{3} \geq 0$, since for $h \rightarrow 0$,

$$
\beta_{2} h^{\beta_{2}}\left|\left[-\int_{0}^{T}\left(\nabla \boldsymbol{U}, \nabla \mathbf{W}_{t}\right) \mathrm{d} s+(\nabla \mathcal{U}(0, \cdot), \nabla \mathbf{W}(0, \cdot))\right]\right| \rightarrow 0,
$$

and for $h \rightarrow 0$,

$$
\beta_{3} h^{\beta_{3}}\left|\int_{0}^{T}\left(\Delta_{h} \mathcal{U}^{+}, \Delta_{h} \mathbf{W}\right) \mathrm{d} s\right| \leq C \beta_{3} h^{\frac{\beta_{3}}{2}}\left(h^{\frac{\beta_{3}}{2}}\left\|\Delta_{h} \mathcal{U}^{+}\right\|_{L^{2}\left(0, T ; \mathbf{L}^{2}\right)}\right)\|\boldsymbol{\zeta}\|_{L^{2}\left(0, T ; \mathbf{L}^{2}\right)} \rightarrow 0 .
$$

Consider now (3.11) 3 , with $\boldsymbol{\psi}(t, \cdot) \equiv \mathbf{R}_{h}^{0} \mathbf{w}(t, \cdot)$ for every $t \in[0, T)$, and every $\mathbf{w} \in C_{0}^{\infty}([0, T), \mathcal{W})$, and integrate the equation over $[0, T]$. We employ integration by parts for the first term to conclude that

$$
\begin{aligned}
-\int_{0}^{T}\left(\mathcal{B}, \boldsymbol{\psi}_{t}\right) \mathrm{d} s+\frac{1}{\bar{\mu}} \int_{0}^{T} \frac{1}{\xi\left(\sigma^{-}\right)}( & \left.\operatorname{curl} \mathcal{B}^{+}, \operatorname{curl} \boldsymbol{\psi}\right) \mathrm{d} s \\
& -\int_{0}^{T}\left(\mathcal{U}^{+} \times \mathcal{B}^{-}, \operatorname{curl} \boldsymbol{\psi}\right) \mathrm{d} s=\left(\mathbf{B}_{0}, \boldsymbol{\psi}(0, \cdot)\right) .
\end{aligned}
$$

Passing to the limit in each of the terms now easily follows from (3.13) 5 , (3.31) (first two terms), (3.31) ${ }_{1}$ (third term), and $\mathbf{B}_{0} \rightarrow \mathbf{b}_{0}$ in $L^{2}(\Omega)$.

The results of this section are summarized in the following

Theorem 3.1. Let $T \equiv k N>0$, and $\mathcal{T}_{h}$ be a strongly acute triangulation of a bounded polyhedral domain $\Omega \subset \mathbb{R}^{3}$, and let (2.8) be valid. Let $(\cdot, \cdot)_{*}=(\cdot, \cdot)_{h}$. Choose $\alpha, \beta_{1}, \beta_{2}, \beta_{3}>0$, such that

(A1) $0<2 \alpha+\beta_{2}<\frac{6-d}{3}, 0<\beta_{1}<2$, and in case $d=3$ and $\alpha \geq \frac{\beta_{3}}{4}$,

(A2) $\sqrt{h}\left\|\nabla \rho^{0}\right\|+\sqrt{\beta_{2}} h^{\frac{\beta_{2}}{2}}\left\|\nabla \mathbf{U}^{0}\right\| \leq C$. 
For every $k, h>0$ sufficiently small, there exists a pentuple

$$
\left\{\left(\mathbf{U}^{n}, P^{n}, \mathbf{B}^{n}, R^{n}, \rho^{n}\right)\right\}_{n=1}^{N} \subset \mathbf{J}_{h} \times L_{h} \times \mathbf{X}_{h} \times S_{h} \times V_{h}
$$

which solves Scheme A, and satisfies (3.4), (3.5), and (3.6). Tending $k, h \rightarrow 0$, where

$$
\rho^{0} \rightarrow \rho_{0} \quad \text { in } L^{2}(\Omega), \quad\left(\mathbf{U}^{0}, \mathbf{B}^{0}\right) \rightarrow\left(\mathbf{u}_{0}, \mathbf{b}_{0}\right) \quad \text { in }\left[\mathbf{L}^{2}(\Omega)\right]^{2},
$$

there exist a convergent subsequence, and

$$
(\mathbf{u}, \mathbf{b}, \rho) \in\left[L^{\infty}(0, T ; \mathbf{H})\right]^{2} \times L^{\infty}\left(0, T ; L^{\infty}(\Omega)\right),
$$

such that

$$
\mathcal{U} \stackrel{*}{\rightarrow} \mathbf{u} \quad \text { in } L^{\infty}\left(0, T ; \mathbf{L}^{2}\right), \mathcal{B} \stackrel{*}{\rightarrow} \mathbf{b} \quad \text { in } L^{\infty}\left(0, T ; \mathbf{L}^{2}\right), \sigma \stackrel{*}{\rightarrow} \rho \text { in } L^{\infty}\left(0, T ; L^{\infty}\right) .
$$

Moreover, $(\mathbf{u}, \mathbf{b}, \rho)$ is a weak solution to (1.1) -(1.2).

Remark 3.1. In 30, a convergent discretization of the incompressible Navier-Stokes equations with variable density and viscosity (i.e., problem (1.1) ${ }_{1-3}$, with $\mathbf{b}=\mathbf{0}$ ) is proposed. In order to balance the conflicting requirements of stability, consistency, and nonnegativity of the density, the key ingredients of the given discontinuous Galerkin scheme are

(i) piecewise constant approximations of the density giving rise to a monotone scheme to validate (3.6),

(ii) velocity fields which have average divergence equal to zero on each element and satisfy $\operatorname{div} \mathcal{U}^{+} \rightarrow 0$ in $L^{2}\left(\Omega_{T}\right)$, which is needed to conclude strong $L^{2}\left(\Omega_{T}\right)$ convergence of approximate densities. A proper scenario [30, Thm. 3.7] are velocity-pressure spaces which satisfy (2.5), and where the pressure space contains piecewise constant functions (Crouzeix-Raviart elements); cf. also [30, Sec. 4].

A complementary scenario is given in this work, where different stabilizing terms are used in the continuous Galerkin method (3.1)-(3.2), and key ingredients of the scheme are

(i') the $M$-matrix property of the stiffness matrix related to (3.1), where element-wise affine, globally continuous density approximations are used. Here, the compatibility assumption (2.8) is needed to balance ansatz spaces of both, pressure and density,

(ii') a stabilization term in (3.2) is inserted to conclude $\operatorname{div} \mathcal{U}^{+} \rightarrow 0$ in $L^{2}\left(\Omega_{T}\right)$. In turn, velocity-pressure spaces which respect (2.5) with continuous pressure functions (e.g., Taylor-Hood elements, MINI element) are admitted. A proper balancing strategy of stabilization parameters given in (A1)-(A2) of Theorem 3.1 is needed to adjust the conflicting discretization and stabilization effects, and hence conclude convergence.

\section{Algorithm A: A simple fixed point scheme to solve Scheme A}

The fully discrete Scheme A requires solving a nonlinear coupled system for every $n \geq 1$. Usually, iterative strategies are employed, which typically go along with a loss of desirable properties for computed iterates, such as the discrete energy identity, and the discrete maximum principle for computed densities in the present case. In fact, the specific iterative scheme has to satisfy a contraction principle to validate convergence to a (unique) limit which solves Scheme A, which is clearly harder to 
satisfy than the requirements needed in Section 3 to validate solvability of Scheme A by Brouwer's fixed point theorem. For practicability, a thresholding criterion has to be supplemented as well to stop the iteration at every time level, which is based on evaluating incremental updates in proper norms. Thresholding criteria have to be properly chosen to make sure that such approximations of solutions to Scheme A eventually converge to weak solutions.

In [33, Section 3], a corresponding program has been realized for the one-fluid magnetohydrodynamics problem, which comes from formally setting $\rho \equiv 1$ in (1.1): it is shown in [33, Theorem 3.2] that sufficient for the proposed iterative scheme to satisfy a contraction property are choices $k \leq \tilde{C} h^{\delta}$, where $\delta=2(d=2)$, and $\delta=4(d=3)$. Clearly, we may not expect a less restrictive mesh constraint in the present case. We draw the following conclusions from [33]:

(i) The nonlinear coupling of $\mathbf{b}$ and $\mathbf{u}$ in equations $(1.1)_{1}$ and $(1.1)_{4}$ is strong, and

(ii) $\mathbf{H}(\mathbf{c u r l} ; \Omega)$-conforming discretizations for the magnetic field have limited stability properties (cf. Lemma 2.1), which is the reason for the above restrictive (sufficient) mesh-constraints to validate the contraction property.

In order to obtain less restrictive mesh constraints which are sufficient for a contraction property in the present context, we propose two changes of Scheme A:

(a) We replace $\mathbf{X}_{h} \subset \mathbf{H}(\mathbf{c u r l} ; \Omega)$ in Scheme A by

$$
\widetilde{\mathbf{X}}_{h}:=\left\{\boldsymbol{\psi} \in \mathbf{C}(\bar{\Omega}): \boldsymbol{\psi} \in \mathbf{P}_{1}(K) \forall K \in \mathcal{T}_{h}\right\} \subset \mathbf{W}^{1,2}(\Omega),
$$

and delete the Lagrange multiplier $R^{n} \in S_{h}$ from this scheme; see e.g. [16, and 11, Sections 3.3 and 3.4]. Note that $\|\mathbf{b}\|_{\mathbf{L}^{6}} \leq C\|\mathbf{b}\|_{\mathbf{W}^{1,2}}$, for all $\mathbf{b} \in \widetilde{\mathbf{X}}_{h}$, as opposed to Lemma 2.1 (ii).

(b) We add the stabilization term $\gamma h^{\gamma}\left(\nabla d_{t} \mathbf{B}^{n}, \nabla \boldsymbol{\psi}\right)$ to the left-hand side of (3.3).

The convergence analysis below holds for convex polyhedral domains.

Remark 4.1. It is immediate to adapt the results in Section 3 to the modified Scheme A, when (a) and (b) hold; Theorem 3.1 remains valid, for $\Omega \subset \mathbb{R}^{3}$ now a convex polyhedron.

The following fixed point algorithm decouples the computation of iterates, and amounts to successively solving three linear problems in every step indexed by $n \geq 1$.

Algorithm A. Choose $\alpha, \beta_{1}, \beta_{2}, \beta_{3}, \gamma>0$, and a threshold parameter $\theta>0$. Let $\left(\rho^{0}, \mathbf{U}^{0}, \mathbf{B}^{0}\right) \in V_{h} \times \mathbf{J}_{h} \times \widetilde{\mathbf{X}}_{h}$, and set $n:=1$.

1. Set $\rho^{n, 0}:=\rho^{n-1}, \mathbf{U}^{n, 0}:=\mathbf{U}^{n-1}, \mathbf{B}^{n, 0}:=\mathbf{B}^{n-1}$, and $\ell=0$. 
2. Let $\ell \geq 1$. Compute $\left(\rho^{n, \ell}, \mathbf{U}^{n, \ell}, P^{n, \ell}, \mathbf{B}^{n, \ell}\right) \in V_{h} \times \mathbf{J}_{h} \times L_{h} \times \widetilde{\mathbf{X}}_{h}$ such that for all $(\chi, \mathbf{W}, \boldsymbol{\psi}) \in V_{h} \times \mathbf{V}_{h} \times \widetilde{\mathbf{X}}_{h}$,

$$
\begin{aligned}
& \left(\frac{\rho^{n, \ell}-\rho^{n-1}}{k}, \chi\right)_{h}+\left(\mathbf{U}^{n, \ell-1} \cdot \nabla \rho^{n, \ell}, \chi\right)+\frac{1}{2}\left(\left[\operatorname{div} \mathbf{U}^{n, \ell-1}\right] \rho^{n, \ell}, \chi\right) \\
& \quad+\alpha h^{\alpha}\left(\nabla \rho^{n, \ell}, \nabla \chi\right)=0, \\
& \frac{1}{2}\left\{\left(\rho^{n-1} \frac{\mathbf{U}^{n, \ell}-\mathbf{U}^{n-1}}{k}, \mathbf{W}\right)_{h}+\left(\frac{\rho^{n, \ell-1} \mathbf{U}^{n, \ell}-\rho^{n-1} \mathbf{U}^{n-1}}{k}, \mathbf{W}\right)_{h}\right. \\
& \quad+\left(\left[\rho^{n-1} \mathbf{U}^{n-1} \cdot \nabla\right] \mathbf{U}^{n, \ell}, \mathbf{W}\right) \\
& \left.\quad-\left(\left[\rho^{n-1} \mathbf{U}^{n-1} \cdot \nabla\right] \mathbf{W}, \mathbf{U}^{n, \ell}\right)\right\}+\left(\eta^{n-1} \mathbf{D}\left(\mathbf{U}^{n, \ell}\right), \boldsymbol{D}(\mathbf{W})\right) \\
& \quad+\beta_{1} h^{-\beta_{1}}\left(\operatorname{div} \mathbf{U}^{n, \ell}, \operatorname{div} \mathbf{W}\right)+\beta_{2} h^{\beta_{2}}\left(\nabla \frac{\mathbf{U}^{n, \ell}-\mathbf{U}^{n-1}}{k}, \nabla \mathbf{W}\right) \\
& \quad+\beta_{3} h^{\beta_{3}}\left(\Delta_{h} \mathbf{U}^{n, \ell}, \Delta_{h} \mathbf{W}\right)-\left(P^{n, \ell}, \operatorname{div} \mathbf{W}\right) \\
& \quad+\frac{1}{\bar{\mu}}\left(\mathbf{B}^{n-1} \times \mathbf{c u r l}^{n, \ell-1}, \mathbf{W}\right)=\left(\rho^{n-1} \mathbf{g}^{n}, \mathbf{W}\right), \\
& \left(\frac{\left.\mathbf{B}^{n, \ell}-\mathbf{B}^{n-1}, \boldsymbol{\psi}\right)+\frac{1}{\bar{\mu}}\left(\frac{1}{\xi^{n-1}} \mathbf{c u r l} \mathbf{B}^{n, \ell}, \mathbf{c u r l} \boldsymbol{\psi}\right)}{k}+\gamma h^{\gamma}\left(\nabla \frac{\mathbf{B}^{n, \ell}-\mathbf{B}^{n-1}}{k}, \nabla \boldsymbol{\psi}\right)\right. \\
& \quad-\left(\mathbf{U}^{n, \ell-1} \times \mathbf{B}^{n-1}, \operatorname{curl} \boldsymbol{\psi}\right)=0 .
\end{aligned}
$$

3. Stop if

$$
\begin{gathered}
\frac{1}{h}\left\|\mathbf{U}^{n, \ell-1}-\mathbf{U}^{n, \ell}\right\|_{h}+\left\|\operatorname{div}\left(\mathbf{U}^{n, \ell-1}-\mathbf{U}^{n, \ell}\right)\right\|+\frac{1}{2 k}\left\|\rho^{n, \ell-1}-\rho^{n, \ell}\right\|_{L^{\infty}} \\
+\frac{1}{\bar{\mu}}\left\|\operatorname{curl}\left[\mathbf{B}^{n, \ell-1}-\mathbf{B}^{n, \ell}\right]\right\|_{\mathbf{L}^{\infty}}+\left\|\mathbf{U}^{n, \ell-1}-\mathbf{U}^{n, \ell}\right\|_{\mathbf{L}^{\infty}} \leq \theta .
\end{gathered}
$$

Set $\rho^{n}:=\rho^{n, \ell+1}, \mathbf{U}^{n}:=\mathbf{U}^{n, \ell+1}, \mathbf{B}^{n}:=\mathbf{B}^{n, \ell+1}$, update $n \leftarrow n+1$ and go to Step 1.

4. Set $\ell \leftarrow \ell+1$, and go to Step 2 .

It will turn out from the subsequent analysis, that choosing $\alpha, \beta_{1}, \beta_{2}, \beta_{3}, \gamma>0$ allows for a discrete maximum principle to hold for positive iterates $\left\{\rho^{n, \ell}\right\}_{\ell \geq 0}$. Moreover, the combination of $\mathbf{W}^{1,2}$-conforming finite elements for the magnetic field $\mathbf{b}$, and stabilization terms only requires a mild mesh constraint (4.5) to validate a contraction principle; at the same time, iterates of Algorithm A $\left\{\left(\rho^{n, \ell}, \mathbf{U}^{n, \ell}, \mathbf{B}^{n, \ell}\right)\right\}_{\ell \geq 0}$ are obtained in a fully decoupled manner. For simplicity, we confine to the case $d=3$.

Theorem 4.1. Let $\Omega \subset \mathbb{R}^{3}$ be a convex polyhedral domain. Suppose that all assumptions in Theorem 3.1 hold, that $\gamma>0$, and $h^{\frac{\gamma}{2}}\left\|\nabla \mathbf{B}^{0}\right\| \leq C$. Let

$$
\left\{\left(\rho^{n, \ell}, \mathbf{U}^{n, \ell}, P^{n, \ell}, \mathbf{B}^{n, \ell}\right)\right\}_{\substack{1 \leq n \leq N \\ \ell \geq 0}}
$$

be a solution to Algorithm A. For every $n \geq 1$, the above mapping to update

$$
\left(\rho^{n, \ell-1}, \mathbf{U}^{n, \ell-1}, \mathbf{B}^{n, \ell-1}\right) \rightarrow\left(\rho^{n, \ell}, \mathbf{U}^{n, \ell}, \mathbf{B}^{n, \ell}\right) \quad(\ell \geq 1)
$$


is a contraction, provided that $k, h>0$ are sufficiently small and satisfy

$$
\tilde{C} k\left[h^{-(1+\gamma)}+h^{-\left(\alpha+3 \beta_{2}\right)}+h^{\alpha-\left(1+2 \beta_{2}\right)}+k h^{-\frac{5}{2} \beta_{2}}+h^{-\left(\frac{\gamma}{2}+\beta_{2}\right)}\right] \leq \frac{1}{2} .
$$

Then, the fixed-point Algorithm A terminates for every $n \geq 1$, and corresponding unique iterates $\left\{\rho^{n, \bar{\ell}_{n}}, \mathbf{U}^{n, \bar{\ell}_{n}}, \mathbf{B}^{n, \bar{\ell}_{n}}\right\} \in V_{h} \times \mathbf{J}_{h} \times \widetilde{\mathbf{X}}_{h}$ satisfy the following perturbed versions of the discrete energy laws:

$$
\begin{aligned}
& \frac{1}{2} \max _{1 \leq n \leq N}\left[\left\|\sqrt{\rho^{n}} \mathbf{U}^{n}\right\|_{h}^{2}+\beta_{2} h^{\beta_{2}}\left\|\nabla \mathbf{U}^{n}\right\|^{2}+\frac{1}{\bar{\mu}}\left\|\mathbf{B}^{n}\right\|^{2}+\gamma h^{\gamma}\left\|\nabla \mathbf{B}^{n}\right\|^{2}\right] \\
& +\frac{k^{2}}{2} \sum_{n=1}^{N}\left[\left\|\sqrt{\rho^{n-1}} d_{t} \mathbf{U}^{n}\right\|_{h}^{2}+\beta_{2} h^{\beta_{2}}\left\|\nabla d_{t} \mathbf{U}^{n}\right\|^{2}\right. \\
& \left.+\left\|d_{t} \mathbf{B}^{n}\right\|^{2}+\gamma h^{\gamma}\left\|\nabla d_{t} \mathbf{B}^{n}\right\|^{2}\right] \\
& +k \sum_{n=1}^{N}\left[\left\|\sqrt{\eta^{n-1}} \boldsymbol{D}\left(\mathbf{U}^{n}\right)\right\|^{2}+\beta_{1} h^{-\beta_{1}}\left\|\operatorname{div} \mathbf{U}^{n}\right\|^{2}+\beta_{3} h^{\beta_{3}}\left\|\Delta_{h} \mathbf{U}^{n}\right\|^{2}\right. \\
& \left.+\frac{1}{\bar{\mu}^{2}}\left\|\frac{1}{\sqrt{\xi^{n-1}}} \operatorname{curl} \mathbf{B}^{n}\right\|^{2}\right] \\
& \leq \frac{1}{2}\left[\left\|\sqrt{\rho^{0}} \mathbf{U}^{0}\right\|_{h}^{2}+\beta_{2} h^{\beta_{2}}\left\|\nabla \mathbf{U}^{0}\right\|^{2}+\frac{1}{\bar{\mu}}\left\|\mathbf{B}^{0}\right\|^{2}+\gamma h^{\gamma}\left\|\nabla \mathbf{B}^{0}\right\|^{2}\right] \\
& +\theta k \sum_{n=1}^{N}\left[\left(\left\|\mathbf{U}^{n}\right\|_{h}+\left\|\mathbf{B}^{n}\right\|+1\right)\left\|\mathbf{U}^{n}\right\|_{h}+\left\|\mathbf{B}^{n-1}\right\|\left\|\operatorname{curl} \mathbf{B}^{n}\right\|\right] \\
& +k \sum_{n=1}^{N}\left|\left(\rho^{n-1} \mathbf{g}^{n}, \mathbf{U}^{n}\right)\right| \\
& \frac{1}{2} \max _{1 \leq n \leq N}\left\|\rho^{n}\right\|_{h}^{2}+\frac{k^{2}}{2} \sum_{n=1}^{N}\left\|d_{t} \rho^{n}\right\|_{h}^{2}+\alpha h^{\alpha} k \sum_{n=1}^{N}\left\|\nabla \rho^{n}\right\|^{2} \\
& \leq \frac{1}{2}\left\|\rho^{0}\right\|_{h}^{2}+C \theta k \sum_{n=1}^{N}\left\|\rho^{n}\right\|,
\end{aligned}
$$

and positive iterates $\left\{\rho^{n, \bar{\ell}_{n}}\right\}_{n} \subset V_{h}$ satisfy the discrete maximum principle (3.6). Moreover,

$$
\left(\rho^{n, \bar{\ell}_{n}}, \mathbf{U}^{n, \bar{\ell}_{n}}, \mathbf{B}^{n, \bar{\ell}_{n}}\right) \rightarrow\left(\sigma^{n}, \mathbf{V}^{n}, \mathbf{C}^{n}\right) \quad(\theta \rightarrow 0)
$$

for every $n \geq 1$, where $\left\{\left(\sigma^{n}, \mathbf{V}^{n}, \mathbf{C}^{n}\right)\right\}_{n=1}^{N} \subset V_{h} \times \mathbf{J}_{h} \times \widetilde{\mathbf{X}}_{h}$ solves Scheme $A$, and overall (subsequence) convergence of $\left\{\left(\rho^{n, \bar{\ell}_{n}}, \mathbf{U}^{n, \bar{\ell}_{n}}, \mathbf{B}^{n, \bar{\ell}_{n}}\right)\right\}_{n=1}^{N}$ towards weak solutions of (1.1)-(1.2) for $k, h, \theta \rightarrow 0$ is valid.

We argue by induction over $n \geq 1$ to verify this result.

Proof. Step 1: Solvability for every $(n, \ell) \geq 0$. We first consider a modified version of Algorithm A, where $\rho^{n, \ell-1}$ in the second term in (4.2) is changed to $\rho_{+}^{n, \ell-1}$.

Let $n \geq 1$, and suppose that $\bar{\rho}_{1} \leq \rho^{n-1} \leq \bar{\rho}_{2}$, and

$$
\left\|\sqrt{\rho^{n-1}} \mathbf{U}^{n-1}\right\|_{h}+\left\|\mathbf{B}^{n-1}\right\|+\sqrt{\beta_{2}} h^{\frac{\beta_{2}}{2}}\left\|\nabla \mathbf{U}^{n-1}\right\|+\sqrt{\gamma} h^{\frac{\gamma}{2}}\left\|\nabla \mathbf{B}^{n-1}\right\| \leq \tilde{C} .
$$


Choose $(\chi, \mathbf{W}, \boldsymbol{\psi})=\left(\rho^{n, \ell}, \mathbf{U}^{n, \ell}, \frac{1}{\bar{\mu}} \mathbf{B}^{n, \ell}\right)$ in (4.1) (4.3) to obtain

$$
\begin{aligned}
\mathcal{X}^{n, \ell}:= & \frac{1}{4 k}\left[\left\|\rho^{n, \ell}\right\|_{h}^{2}+\left\|\sqrt{\rho^{n-1}} \mathbf{U}^{n, \ell}\right\|_{h}^{2}+\left\|\sqrt{\rho_{+}^{n, \ell-1}} \mathbf{U}^{n, \ell}\right\|_{h}^{2}\right. \\
& \left.+\beta_{2} h^{\beta_{2}}\left\|\nabla \mathbf{U}^{n, \ell}\right\|^{2}+\frac{1}{\bar{\mu}}\left\|\mathbf{B}^{n, \ell}\right\|^{2}+\gamma h^{\gamma}\left\|\nabla \mathbf{B}^{n, \ell}\right\|^{2}\right]+\alpha h^{\alpha}\left\|\nabla \rho^{n, \ell}\right\|^{2} \\
& +\beta_{3} h^{\beta_{3}}\left\|\Delta_{h} \mathbf{U}^{n, \ell}\right\|^{2}+\beta_{1} k^{-\beta_{1}}\left\|\operatorname{div} \mathbf{U}^{n, \ell}\right\|^{2}+\left\|\sqrt{\eta^{n-1}} \boldsymbol{D}\left(\mathbf{U}^{n, \ell}\right)\right\|^{2} \\
& +\frac{1}{\bar{\mu}^{2}}\left\|\frac{1}{\sqrt{\xi^{n-1}}} \operatorname{curl} \mathbf{B}^{n, \ell}\right\|^{2} \\
\leq & \frac{1}{k}\left[\left\|\rho^{n-1}\right\|_{h}^{2}+\left\|\sqrt{\rho^{n-1}} \mathbf{U}^{n-1}\right\|_{h}^{2}+\beta_{2} h^{\beta_{2}}\left\|\nabla \mathbf{U}^{n-1}\right\|^{2}+\frac{1}{\bar{\mu}}\left\|\mathbf{B}^{n-1}\right\|^{2}\right]-\frac{1}{\bar{\mu}} I,
\end{aligned}
$$

where, by inverse inequality, (2.3) and (4.8),

$$
\begin{aligned}
I:= & \left(\mathbf{B}^{n-1} \times \mathbf{c u r l} \mathbf{B}^{n, \ell-1}, \mathbf{U}^{n, \ell}\right)+\left(\mathbf{U}^{n, \ell-1} \times \mathbf{B}^{n-1}, \operatorname{curl} \mathbf{B}^{n, \ell}\right) \\
\leq & C h^{-1}\left\|\mathbf{B}^{n-1}\right\|_{\mathbf{L}^{6}}^{2}\left[\frac{k}{\bar{\rho}_{1}}\left\|\operatorname{curl}^{n, \ell-1}\right\|^{2}+\bar{\xi}_{+} \bar{\mu}^{2}\left\|\mathbf{U}^{n, \ell-1}\right\|_{h}^{2}\right] \\
& +\frac{1}{2}\left[\frac{1}{4 k}\left\|\sqrt{\rho^{n-1}} \mathbf{U}^{n, \ell}\right\|_{h}^{2}+\frac{1}{\bar{\mu}^{2}}\left\|\frac{1}{\sqrt{\xi^{n-1}}} \operatorname{curl~B}^{n, \ell}\right\|^{2}\right] \\
\leq & C h^{-(1+\gamma)}\left[\frac{k}{\bar{\rho}_{1}}\left\|\operatorname{curl} \mathbf{B}^{n, \ell-1}\right\|^{2}+\bar{\mu}^{2} \bar{\xi}_{+}\left\|\mathbf{U}^{n, \ell-1}\right\|_{h}^{2}\right] \\
& +\frac{1}{2}\left[\frac{1}{4 k}\left\|\sqrt{\rho^{n-1}} \mathbf{U}^{n, \ell}\right\|_{h}^{2}+\frac{1}{\bar{\mu}^{2}}\left\|\frac{1}{\sqrt{\xi^{n-1}}} \operatorname{curlB}^{n, \ell}\right\|^{2}\right] .
\end{aligned}
$$

In order to validate $\frac{1}{2} \mathcal{X}^{n, \ell} \leq \frac{1}{k} \tilde{C}+\frac{1}{4} \mathcal{X}^{n, \ell-1}$ for all $\ell \geq 1$, we have to make sure that

$$
C h^{-(1+\gamma)} \frac{k}{\bar{\rho}_{1}} \leq \frac{1}{16 \bar{\mu}^{2} \bar{\xi}_{-}} \quad \text { and } \quad C h^{-(1+\gamma)} \bar{\mu}^{2} \bar{\xi}_{+} \leq \frac{\bar{\rho}_{1}}{16 k} .
$$

Then, we may conclude recursively that for all $\ell \geq 1$,

$$
\begin{gathered}
\left\|\rho^{n, \ell}\right\|_{h}^{2}+\left\|\sqrt{\rho^{n-1}} \mathbf{U}^{n, \ell}\right\|_{h}^{2}+\left\|\sqrt{\rho_{+}^{n, \ell-1}} \mathbf{U}^{n, \ell}\right\|_{h}^{2}+\beta_{2} h^{\beta_{2}}\left\|\nabla \mathbf{U}^{n, \ell}\right\|^{2} \\
+\gamma h^{\gamma}\left\|\nabla \mathbf{B}^{n, \ell}\right\|^{2}+\frac{1}{\bar{\mu}}\left\|\mathbf{B}^{n, \ell}\right\|^{2} \\
+k\left[\alpha h^{\alpha}\left\|\nabla \rho^{n, \ell}\right\|^{2}+\left\|\sqrt{\eta^{n-1}} \boldsymbol{D}\left(\mathbf{U}^{n, \ell}\right)\right\|^{2}+\frac{1}{\bar{\mu}}\left\|\frac{1}{\sqrt{\xi^{n-1}}} \operatorname{curl}^{n, \ell}\right\|^{2}\right. \\
\left.+\beta_{1} h^{-\beta_{1}}\left\|\operatorname{div} \mathbf{U}^{n, \ell}\right\|^{2}+\beta_{3} h^{\beta_{3}}\left\|\Delta_{h} \mathbf{U}^{n, \ell}\right\|^{2}\right] \leq 16 \tilde{C},
\end{gathered}
$$

where $\tilde{C}>0$ is the constant used in (4.8). This result also validates existence of unique solutions for Algorithm A by Lax-Milgram theorem.

Step 2: Positivity of density iterates, and discrete maximum principle for every $(n, \ell) \geq 0$. It holds that

$$
\bar{\rho}_{1} \leq \rho^{n, \ell} \leq \bar{\rho}_{2} \quad \forall(n, \ell) \geq 0 .
$$

This property can be shown as in Step 3 of the proof of Lemma 3.1, by using (4.9).

As a consequence, we may drop the initial change of the second term in (4.2) in Step 1 , where $\rho^{n, \ell-1}$ was replaced by $\rho_{+}^{n, \ell-1}$ to verify (4.9). 
Step 3: Contraction property. Fix $n \geq 1$. Let $\mathbf{E}_{\varphi}^{n, \ell}:=\varphi^{n, \ell}-\varphi^{n, \ell-1}$, for $\varphi \in$ $\{\mathbf{U}, \mathbf{B}, P, \rho\}$, and suppose that $\bar{\rho}_{1} \leq \rho^{n-1} \leq \bar{\rho}_{2}$, as well as (4.8). Then, for all $(\chi, \mathbf{W}, \boldsymbol{\psi}) \in V_{h} \times \mathbf{J}_{h} \times \widetilde{\mathbf{X}}_{h}$,

$$
\begin{aligned}
& \frac{1}{k}\left(E_{\rho}^{n, \ell}, \chi\right)_{h}+\left(\mathbf{U}^{n, \ell-2} \cdot \nabla E_{\rho}^{n, \ell}, \chi\right) \\
& \quad+\frac{1}{2}\left(\left[\operatorname{div} \mathbf{U}^{n, \ell-2}\right] E_{\rho}^{n, \ell}, \chi\right)+\alpha h^{\alpha}\left(\nabla E_{\rho}^{n, \ell}, \nabla \chi\right) \\
& =-\left(\mathbf{E}_{\mathbf{U}}^{n, \ell-1} \nabla \rho^{n, \ell}, \chi\right)-\frac{1}{2}\left(\left[\operatorname{div} \mathbf{E}_{\mathbf{U}}^{n, \ell-1}\right] \rho^{n, \ell}, \chi\right) \\
& \frac{1}{2}\left\{\frac{1}{k}\left(\rho^{n-1} \mathbf{E}_{\mathbf{U}}^{n, \ell}, \mathbf{W}\right)_{h}+\frac{1}{k}\left(\rho^{n, \ell-2} \mathbf{E}_{\mathbf{U}}^{n, \ell}+E_{\rho}^{n, \ell-1} \mathbf{U}^{n, \ell}, \mathbf{W}\right)_{h}\right. \\
& \quad+\left(\left[\rho^{n-1} \mathbf{U}^{n-1} \cdot \nabla\right] \mathbf{E}_{\mathbf{U}}^{n, \ell}, \mathbf{W}\right) \\
& \left.\quad-\left(\left[\rho^{n-1} \mathbf{U}^{n-1} \cdot \nabla\right] \mathbf{W}, \mathbf{E}_{\mathbf{U}}^{n, \ell}\right)\right\}-\left(E_{P}^{n, \ell}, \operatorname{div} \mathbf{W}\right) \\
& \quad+\beta_{1} h^{-\beta_{1}}\left(\operatorname{div} \mathbf{E}_{\mathbf{U}}^{n, \ell}, \operatorname{div} \mathbf{W}\right)+\beta_{3}\left(\Delta_{h} \mathbf{E}_{\mathbf{U}}^{n, \ell}, \Delta_{h} \mathbf{W}\right) \\
& \quad+\left(\eta^{n-1} \mathbf{D}\left(\mathbf{E}_{\mathbf{U}}^{n, \ell}\right), \mathbf{D}(\mathbf{W})\right)+\beta_{2} \frac{h^{\beta_{2}}}{k}\left(\nabla \mathbf{E}_{\mathbf{U}}^{n, \ell}, \nabla \mathbf{W}\right) \\
& \quad+\frac{1}{\bar{\mu}}\left(\mathbf{B}^{n-1} \times \mathbf{c u r l}^{n, \ell-1}, \mathbf{W}\right)=0, \\
& \frac{1}{k}\left(\mathbf{E}_{\mathbf{B}}^{n, \ell}, \boldsymbol{\psi}\right)+\frac{1}{\bar{\mu}}\left(\frac{1}{\xi^{n-1}} \mathbf{c u r l} \mathbf{E}_{\mathbf{B}}^{n, \ell}, \mathbf{c u r l} \psi\right)+\frac{\gamma h^{\gamma}}{k}\left(\nabla \mathbf{E}_{\mathbf{B}}^{n, \ell}, \nabla \boldsymbol{\psi}\right) \\
& \quad-\left(\mathbf{E}_{\mathbf{U}}^{n, \ell-1} \times \mathbf{B}^{n-1}, \operatorname{curl} \boldsymbol{\psi}\right)=0 .
\end{aligned}
$$

Choose $(\chi, \mathbf{W}, \boldsymbol{\psi})=\left(E_{\rho}^{n, \ell}, \mathbf{E}_{\mathbf{U}}^{n, \ell}, \mathbf{E}_{\mathbf{B}}^{n, \ell}\right)$ to obtain

$$
\begin{aligned}
\frac{1}{k}\{ & \left\|E_{\rho}^{n, \ell}\right\|_{h}^{2}+\frac{1}{2}\left\|\sqrt{\rho^{n-1}} \mathbf{E}_{\mathbf{U}}^{n, \ell}\right\|_{h}^{2}+\frac{1}{2}\left\|\sqrt{\rho^{n, \ell-2}} \mathbf{E}_{\mathbf{U}}^{n, \ell}\right\|_{h}^{2}+\left\|\mathbf{E}_{\mathbf{B}}^{n, \ell}\right\|^{2} \\
& \left.+\beta_{2} h^{\beta_{2}}\left\|\nabla \mathbf{E}_{\mathbf{U}}^{n, \ell}\right\|^{2}+\gamma h^{\gamma}\left\|\nabla \mathbf{E}_{\mathbf{B}}^{n, \ell}\right\|^{2}\right\}+\alpha h^{\alpha}\left\|\nabla E_{\rho}^{n, \ell}\right\|^{2}+\bar{\eta}_{-}\left\|\boldsymbol{D}\left(\mathbf{E}_{\mathbf{U}}^{n, \ell}\right)\right\|^{2} \\
& +\frac{1}{\bar{\mu}}\left\|\frac{1}{\sqrt{\xi^{n-1}}} \operatorname{curl} \mathbf{E}_{\mathbf{B}}^{n, \ell}\right\|^{2}+\beta_{1} h^{-\beta_{1}}\left\|\operatorname{div} \mathbf{E}_{\mathbf{U}}^{n, \ell}\right\|^{2}+\beta_{3} h^{\beta_{3}}\left\|\Delta_{h} \mathbf{E}_{\mathbf{U}}^{n, \ell}\right\|^{2} \\
\leq & C\left(\left\|\mathbf{E}_{\mathbf{U}}^{n, \ell-1}\right\|\left\|\nabla \rho^{n, \ell}\right\|_{L^{\infty}}\left\|E_{\rho}^{n, \ell}\right\|+\left\|\nabla \mathbf{E}_{\mathbf{U}}^{n, \ell-1}\right\|\left\|\rho^{n, \ell}\right\|_{L^{\infty}}\left\|E_{\rho}^{n, \ell}\right\|\right) \\
& +C \bar{\xi}_{+} \bar{\mu}\left\|\mathbf{B}^{n-1}\right\|_{\mathbf{L}^{3}}^{2}\left(\left\|\nabla \mathbf{E}_{\mathbf{U}}^{n, \ell}\right\|_{\mathbf{L}^{2}}^{2}+\left\|\nabla \mathbf{E}_{\mathbf{U}}^{n, \ell-1}\right\|_{\mathbf{L}^{2}}^{2}\right) \\
& +\frac{1}{8 \bar{\mu}}\left\|\frac{1}{\sqrt{\xi^{n-1}}} \operatorname{curr}_{\mathbf{B}}^{n, \ell-1}\right\|^{2}+\frac{1}{2 \bar{\mu}}\left\|\frac{1}{\sqrt{\xi^{n-1}}} \operatorname{curl}_{\mathbf{B}} \mathbf{E}_{\mathbf{B}}^{n, \ell}\right\|^{2} \\
& -\frac{1}{2 k}\left(E_{\rho}^{n, \ell-1} \mathbf{U}^{n, \ell}, \mathbf{E}_{\mathbf{U}}^{n, \ell}\right)_{h} .
\end{aligned}
$$

A critical term is the last one, which is the main motivation to use mass lumping for the leading two terms in (4.2), which allows to benefit from (4.11) 1 directly. However, we remark that this is not necessary for the arguments to follow, and exact integration of the leading two terms in (4.2) could be treated as well by the following argument: additional terms could then be controlled by (2.4), together with derivative terms on the left-hand side of (4.12). 
We use (4.11) 1 to bound the last term as

$$
\begin{aligned}
-\frac{1}{k} & \left(E_{\rho}^{n, \ell-1},\left\langle\mathbf{U}^{n, \ell}, \mathbf{E}_{\mathbf{U}}^{n, \ell}\right\rangle\right)_{h}=\left(\left\langle\mathbf{U}^{n, \ell-3}, \nabla E_{\rho}^{n, \ell-1}\right\rangle, \mathcal{I}_{V_{h}}\left\langle\mathbf{U}^{n, \ell}, \mathbf{E}_{\mathbf{U}}^{n, \ell}\right\rangle\right) \\
& +\frac{1}{2}\left(\left[\operatorname{div} \mathbf{U}^{n, \ell-3}\right] E_{\rho}^{n, \ell-1}, \mathcal{I}_{V_{h}}\left\langle\mathbf{U}^{n, \ell}, \mathbf{E}_{\mathbf{U}}^{n, \ell}\right\rangle\right) \\
& +\alpha h^{\alpha}\left(\nabla E_{\rho}^{n, \ell-1}, \nabla \mathcal{I}_{V_{h}}\left\langle\mathbf{U}^{n, \ell}, \mathbf{E}_{\mathbf{U}}^{n, \ell}\right\rangle\right) \\
& +\left(\mathbf{E}_{\mathbf{U}}^{n, \ell-2} \nabla \rho^{n, \ell-1}, \mathcal{I}_{V_{h}}\left\langle\mathbf{U}^{n, \ell}, \mathbf{E}_{\mathbf{U}}^{n, \ell}\right\rangle\right) \\
& +\frac{1}{2}\left(\left[\operatorname{div} \mathbf{E}_{\mathbf{U}}^{n, \ell-2}\right] \rho^{n, \ell-1}, \mathcal{I}_{V_{h}}\left\langle\mathbf{U}^{n, \ell}, \mathbf{E}_{\mathbf{U}}^{n, \ell}\right\rangle\right) \\
\leq & C\left\|\nabla \mathbf{U}^{n, \ell-3}\right\|\left\|\nabla E_{\rho}^{n, \ell-1}\right\|\left\|\nabla \mathbf{U}^{n, \ell}\right\|\left\|\nabla \mathbf{E}_{\mathbf{U}}^{n, \ell}\right\| \\
& +\alpha h^{\alpha}\left\|\nabla E_{\rho}^{n, \ell-1}\right\|_{\mathbf{L}^{2}}\left(\left\|\nabla \mathbf{U}^{n, \ell}\right\|_{\mathbf{L}^{2}}\left\|\mathbf{E}_{\mathbf{U}}^{n, \ell}\right\|_{\mathbf{L}^{\infty}}+\left\|\mathbf{U}^{n, \ell}\right\|_{\mathbf{L} \infty}\left\|\nabla \mathbf{E}_{\mathbf{U}}^{n, \ell}\right\|_{\mathbf{L}^{2}}\right) \\
& +C \bar{\rho}_{1}^{-\frac{1}{4}} h^{-\left(\frac{\beta_{2}}{4}+2 \frac{\beta_{2}}{2}\right)}\left\|\sqrt{\rho^{n-1}} \mathbf{U}^{n, \ell}\right\|^{1 / 2}\left(h^{\frac{\beta_{2}}{4}}\left\|\nabla \mathbf{U}^{n, \ell}\right\|^{1 / 2}\right) \\
& \left(h^{\frac{\beta_{2}}{2}}\left\|\nabla \mathbf{E}_{\mathbf{U}}^{n, \ell-2}\right\|\right)\left(h^{\frac{\beta_{2}}{2}}\left\|\nabla \mathbf{E}_{\mathbf{U}}^{n, \ell}\right\|\right),
\end{aligned}
$$

thanks to (4.10), the continuous embedding $W^{1,2} \hookrightarrow L^{6}$, and interpolation of $L^{3}$ between $L^{2}$ and $W^{1,2}$, as well as integration by parts in the last but one term in front of the inequality sign, and inverse estimates. We may then proceed by

$$
\begin{aligned}
\leq & C h^{-3 \frac{\beta_{2}}{2}-\frac{\alpha}{2}}\left(h^{\frac{\beta_{2}}{2}}\left\|\nabla \mathbf{U}^{n, \ell-3}\right\|\right)\left(h^{\frac{\alpha}{2}}\left\|\nabla E_{\rho}^{n, \ell-1}\right\|\right)\left(h^{\frac{\beta_{2}}{2}}\left\|\nabla \mathbf{U}^{n, \ell}\right\|\right)\left(h^{\frac{\beta_{2}}{2}}\left\|\nabla \mathbf{E}_{\mathbf{U}}^{n, \ell}\right\|\right) \\
& +\alpha h^{\frac{\alpha}{2}-2 \frac{\beta_{2}}{2}-\frac{1}{2}}\left(h^{\frac{\alpha}{2}}\left\|\nabla E_{\rho}^{n, \ell-1}\right\|\right)\left(h^{\frac{\beta_{2}}{2}}\left\|\nabla \mathbf{U}^{n, \ell}\right\|\right)\left(h^{\frac{\beta_{2}}{2}}\left\|\nabla \mathbf{E}_{\mathbf{U}}^{n, \ell}\right\|\right) \\
& +C \bar{\rho}_{1}^{-\frac{1}{4}} h^{-\left(\frac{\beta_{2}}{4}+2 \frac{\beta_{2}}{2}\right)}\left\|\sqrt{\rho^{n-1}} \mathbf{U}^{n, \ell}\right\|^{3 / 2}\left(h^{\frac{\beta_{2}}{4}}\left\|\nabla \mathbf{U}^{n, \ell}\right\|^{1 / 2}\right) \\
& \left(h^{\frac{\beta_{2}}{2}}\left\|\nabla \mathbf{E}_{\mathbf{U}}^{n, \ell-2}\right\|\right)\left(h^{\frac{\beta_{2}}{2}}\left\|\nabla \mathbf{E}_{\mathbf{U}}^{n, \ell}\right\|\right) .
\end{aligned}
$$

We employ (4.9) to conclude that

$$
\begin{aligned}
\leq C & {\left[h^{-3 \beta_{2}-\alpha}+h^{\alpha-1-2 \beta_{2}}\right]\left(h^{\alpha}\left\|\nabla E_{\rho}^{n, \ell-1}\right\|^{2}\right)+\frac{1}{2 k} \beta_{2} h^{\beta_{2}}\left\|\nabla \mathbf{E}_{\mathbf{U}}^{n, \ell}\right\|^{2} } \\
& +C k h^{-\frac{5}{2} \beta_{2}}\left(h^{\beta_{2}}\left\|\nabla \mathbf{E}_{\mathbf{U}}^{n, \ell-2}\right\|^{2}\right) .
\end{aligned}
$$

By using this estimate in (4.12) requires

$$
C k\left[h^{-3 \beta_{2}-\alpha}+h^{\alpha-1-2 \beta_{2}}+h^{-\frac{5}{2} \beta_{2}}\right] \leq \frac{1}{2}
$$

to allow for a contraction property. For the first two terms on the right-hand side of (4.12), we find an upper bound

$$
\leq C h^{-1}\left(\left\|\mathbf{E}_{\mathbf{U}}^{n, \ell-1}\right\|_{h}^{2}+\left\|E_{\rho}^{n, \ell}\right\|^{2}\right)+\frac{\bar{\eta}}{4}\left\|\boldsymbol{D}\left(\mathbf{E}_{\mathbf{U}}^{n, \ell}\right)\right\|^{2}+C\left\|E_{\rho}^{n, \ell}\right\|_{h}^{2},
$$

which requires discretization parameters $k, h \geq 0$ such that

$$
k \leq C h
$$

to validate the contraction property.

For the third term on the right-hand side of (4.12), we obtain as an upper bound

$$
\leq C h^{-\frac{\gamma}{2}-\beta_{2}}\left\|\mathbf{B}^{n-1}\right\|\left(h^{\frac{\gamma}{2}}\left\|\nabla \mathbf{B}^{n-1}\right\|\right)\left[\left(\beta_{2} h^{\beta_{2}}\left\|\nabla \mathbf{E}_{\mathbf{U}}^{n, \ell}\right\|^{2}\right)+\left(\beta_{2} h^{\beta_{2}}\left\|\nabla \mathbf{E}_{\mathbf{U}}^{n, \ell-1}\right\|^{2}\right)\right] .
$$


Similarly, a sufficient condition to obtain the contraction property is

$$
C k h^{-\left(\frac{\gamma}{2}+\beta_{2}\right)} \leq \frac{1}{2}
$$

Putting things together in (4.12) yields the contraction property of Algorithm A for values $k, h>0$ which satisfy (4.14), (4.13), (4.15).

Step 4: Overall convergence. The previous step shows that Algorithm A terminates. Hence, for every $\theta>0$, and every $n \geq 1$, there exists $\bar{\ell}_{n}:=\ell(n, \theta)<\infty$, such that the stopping criterion is met. In particular, solutions $\left\{\left(\rho^{n}, \mathbf{U}^{n}, P^{n}, \mathbf{B}^{n}\right)\right\}_{n=1}^{N}$, with each

$$
\rho^{n}:=\rho^{n, \bar{\ell}_{n}}, \quad \mathbf{U}^{n}:=\mathbf{U}^{n, \bar{\ell}_{n}}, \quad P^{n}:=P^{n, \bar{\ell}_{n}}, \quad \mathbf{B}^{n}:=\mathbf{B}^{n, \bar{\ell}_{n}}
$$

satisfy Scheme A, with modified right-hand sides in (3.1):

$$
\begin{gathered}
\left(\left[\mathbf{U}^{n}-\mathbf{U}^{n, \bar{\ell}_{n}-1}\right] \cdot \nabla \rho^{n}, \chi\right)+\frac{1}{2}\left(\left[\operatorname{div}\left(\mathbf{U}^{n}-\mathbf{U}^{n, \bar{\ell}_{n}-1}\right)\right] \rho^{n}, \chi\right), \\
\leq\left(C h^{-1}\left\|\mathbf{U}^{n}-\mathbf{U}^{n, \bar{\ell}_{n}-1}\right\|+\left\|\operatorname{div}\left(\mathbf{U}^{n}-\mathbf{U}^{n, \bar{\ell}_{n}-1}\right)\right\|\right)\|\chi\| ;
\end{gathered}
$$

in (3.2):

$$
\begin{aligned}
& \frac{1}{2 k}\left(\left[\rho^{n}-\rho^{n, \bar{\ell}_{n}-1}\right] \mathbf{U}^{n}, \mathbf{W}\right)+\left(\mathbf{B}^{n-1} \times \mathbf{c u r l}\left[\mathbf{B}^{n}-\mathbf{B}^{n, \bar{\ell}_{n}-1}\right], \mathbf{W}\right) \\
& \quad \leq\left[\frac{1}{2 k}\left\|\rho^{n}-\rho^{n, \bar{\ell}_{n}-1}\right\|_{L^{\infty}}\left\|\mathbf{U}^{n}\right\|+\left\|\mathbf{c u r l}\left(\mathbf{B}^{n}-\mathbf{B}^{n, \bar{\ell}_{n}-1}\right)\right\|_{\mathbf{L} \infty}\left\|\mathbf{B}^{n-1}\right\|\right]\|\mathbf{W}\|,
\end{aligned}
$$

and

$$
\begin{gathered}
\left(d_{t} \mathbf{B}^{n}, \boldsymbol{\psi}\right)+\frac{1}{\bar{\mu}}\left(\frac{1}{\xi^{n-1}} \operatorname{curl}^{n}, \operatorname{curl} \boldsymbol{\psi}\right)+\gamma h^{\gamma}\left(\nabla d_{t} \mathbf{B}^{n}, \nabla \boldsymbol{\psi}\right) \\
\quad-\left(\mathbf{U}^{n} \times \mathbf{B}^{n-1}, \operatorname{curl} \boldsymbol{\psi}\right) \\
=\left(\left[\mathbf{U}^{n}-\mathbf{U}^{n, \bar{\ell}_{n}-1}\right] \times \mathbf{B}^{n-1}, \operatorname{curl} \boldsymbol{\psi}\right)
\end{gathered}
$$

where the right-hand side may be bounded by $\left\|\mathbf{U}^{n}-\mathbf{U}^{n, \bar{\ell}_{n}-1}\right\|_{\mathbf{L}^{\infty}}\left\|\mathbf{B}^{n-1}\right\|\|\mathbf{c u r l} \boldsymbol{\psi}\|$. The remaining assertions of the theorem now easily follow from the results in Section 3, in particular, the perturbed energy estimates (4.6), (4.7) hold, which justifies assumption (4.8) in Step 1, for some finite $\tilde{C} \equiv \tilde{C}(T)>0$.

\section{Computational experiments}

In this section we give details about the practical implementation of the numerical method analyzed in the previous sections and present a number of computations in $3 \mathrm{D}$.

5.1. Numerical implementation. If not mentioned otherwise, the following physical parameters were constants in our experiments: the viscosity $\eta=10^{-3}$, the permeability $\bar{\mu}=\mu_{0}=1.25 \times 10^{-6}$, and the gravitational acceleration $\mathbf{g}=$ $(0,0,-10)$. Given the physical densities of the two respective fluids $\bar{\rho}_{1}, \bar{\rho}_{2}$, we define $\bar{\rho}_{\max }=\max \left\{\bar{\rho}_{1}, \bar{\rho}_{2}\right\}$, and denote nondimensional densities $\tilde{\rho}_{1}=\bar{\rho}_{1} / \bar{\rho}_{\max }$, $\tilde{\rho}_{2}=\bar{\rho}_{2} / \bar{\rho}_{\max }$. The nondimensional mean density is defined as $\tilde{\rho}_{*}=0.5\left(\tilde{\rho}_{1}+\tilde{\rho}_{2}\right)$. For computational convenience the density and the pressure are scaled in the following text without explicit change of the notation, i.e., $\rho^{n} \equiv \rho^{n} / \bar{\rho}_{\max }$ and $P^{n} \equiv P^{n} / \bar{\rho}_{\max }$, $n \geq 0$. As we use locally adapted unstructured meshes in some of the numerical 
experiments, by $h$ we understand an elementwise constant mesh size indicator function $\left.h(\mathbf{x})\right|_{K}=h_{K}$, where $h_{K}$ denotes the diameter of the mesh element $K \in \mathcal{T}_{h}$. Further, we allow for nonuniform time steps, and implicitly assume that $k \equiv k^{n}$ on a given time level $n$.

5.1.1. Navier-Stokes equations. We employ the following stabilized variational formulation for the solution of the Navier-Stokes equations in the numerical experiments

$$
\begin{aligned}
\frac{1}{2}\left\{\left(\rho^{n-1} \frac{\mathbf{U}^{n, \ell}-\mathbf{U}^{n-1}}{k}, \mathbf{W}\right)+\left(\frac{\rho^{n, \ell-1} \mathbf{U}^{n, \ell}-\rho^{n-1} \mathbf{U}^{n-1}}{k}, \mathbf{W}\right)\right. \\
\left.\quad+\left(\left[\rho^{n-1} \mathbf{U}^{n-1} \cdot \nabla\right] \mathbf{U}^{n, \ell}, \mathbf{W}\right)-\left(\left[\rho^{n-1} \mathbf{U}^{n-1} \cdot \nabla\right] \mathbf{W}, \mathbf{U}^{n, \ell}\right)\right\} \\
\quad+\frac{\eta}{\bar{\rho}_{\max }}\left(\boldsymbol{D}\left(\mathbf{U}^{n, \ell}\right), \boldsymbol{D}(\mathbf{W})\right)+\left(\frac{h}{k} \nabla\left(\mathbf{U}^{n, \ell}-\mathbf{U}^{n-1}\right), \nabla \mathbf{W}\right) \\
\quad+\frac{1}{\bar{\mu} \bar{\rho}_{\max }}\left(\mathbf{B}^{n-1} \times \mathbf{c u r l} \mathbf{B}^{n, \ell-1}, \mathbf{W}\right)-\left(P^{n, \ell}, \operatorname{div} \mathbf{W}\right) \\
=\left(\rho^{n, \ell-1} \mathbf{g}^{n}, \mathbf{W}\right)\left(\operatorname{div} \mathbf{U}^{n, \ell}, \varphi\right)=0,
\end{aligned}
$$

with $\mathbf{W} \in \mathbf{V}_{h}, \varphi \in V_{h}$. We used a $P 2 / P 1$ Taylor-Hood finite element method for the approximation of the above variational formulation, i.e., continuous piecewise quadratic finite element functions for the velocity, and continuous piecewise affine functions for the pressure. We found that the inclusion of the stabilization term $\left(\frac{h}{k} \nabla\left(\mathbf{U}^{n, \ell}-\mathbf{U}^{n-1}\right), \nabla \mathbf{W}\right)$ in the above formulation (i.e., the $\beta_{2}$-term from (4.2) with $\beta_{2}=1$ ) was essential to obtain a solution without spurious oscillations; see also Remark 5.1 .

We consider two types of boundary conditions for the velocity field, the homogeneous Dirichlet boundary condition ("no slip") and a "slip" boundary condition

$$
\mathbf{U} \cdot \mathbf{n}=0 \quad \text { and } \quad \frac{\eta}{\bar{\rho}_{\max }}(\boldsymbol{D}(\mathbf{U}) \mathbf{n}-P \mathbf{n}) \cdot \mathbf{t}_{i}=0, \quad i=1,2 \quad \text { on } \quad \partial \Omega,
$$

where $\mathbf{t}_{i}, i=1,2$ are two unit vectors orthogonal to $\mathbf{n}$.

Remark 5.1 (Stabilization of the Navier-Stokes equations). In general, some sort of stabilization is necessary to obtain a meaningful numerical solution of the NavierStokes equations. The $\beta_{1}$ and $\beta_{3}$ stabilization terms in (3.2) and (4.2) are included mainly for theoretical reasons. The $\beta_{1}$ and $\beta_{3}$ terms have similar counterparts in more established SUPG-type stabilization techniques; cf., e.g. [10]. In practice all stabilization contributions need to be carefully balanced, which is nontrivial in the present setting, and exceeds the scope of the present work. We have obtained satisfactory results with the formulation (5.1) which only includes the $\beta_{2}$ stabilization term from (4.2). For simplicity, we have not considered the remaining $\beta_{1}$ and $\beta_{3}$ terms in our computations and conclude that further numerical tests are needed to investigate this issue.

The saddle point system corresponding to (5.1) takes the following form:

$$
\left(\begin{array}{cc}
A & B \\
C & 0
\end{array}\right)\left(\frac{U}{\underline{P}}\right)=\left(\frac{f}{\underline{g}}\right) \text {. }
$$

We solve the above linear system by a multigrid method with a Vanka-type smoother. Below, we give a brief description of the method, for more details about Vankatype multigrid solvers for Navier-Stokes equations see, e.g., 222 and the references 

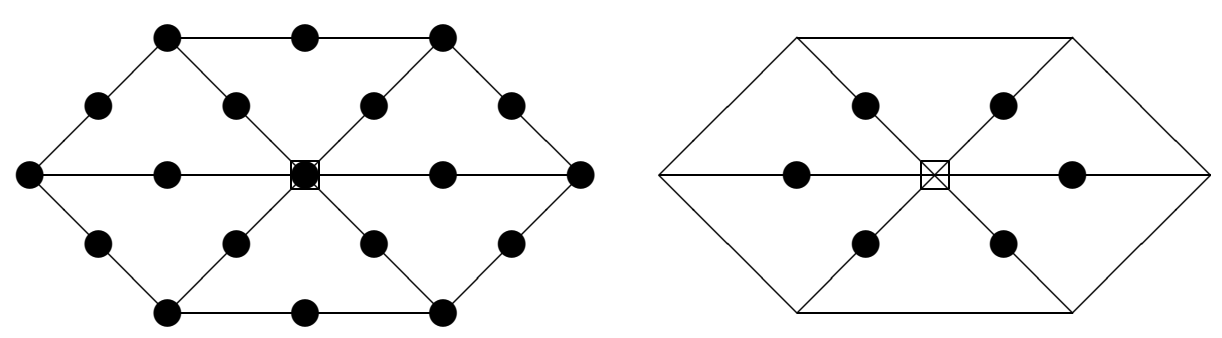

FiguRE 1. Local smoothing patches in 2D for the Vanka smoother (left) and the edge element smoother (right); degrees of freedom

$(\bullet)$ associated with the vertex $(\square)$.

therein; for an introduction to multigrid methods see, e.g. 20]. A general multigrid method consists of four basic components: a grid hierarchy, grid transfer operators, a smoother, and a coarse grid solver. The hierarchy of nested grids is constructed automatically from a given macro triangulation by recursive mesh refinement and coarsening routines included in the finite element package Albert [34 that provides a base for our finite element code. The grid transfer operators are induced by the natural embedding of the finite elements spaces associated with the grid hierarchy. A multigrid smoother is usually a Gauss-Seidel type iteration, however the standard Gauss-Seidel method cannot be applied directly to the system (5.3). Vanka-type smoothers can be considered as block Gauss-Seidel methods with local blocks

$$
\left(\begin{array}{cc}
A_{j} & B_{j} \\
C_{j} & 0
\end{array}\right)\left(\frac{U}{\underline{P}}\right)_{j}=\left(\frac{f}{\underline{g}}\right)_{j}
$$

where the vector of local unknowns $(\underline{U}, \underline{P})_{j}$ consists of one pressure degree of free$\operatorname{dom}\{\underline{P}\}_{j}$ and all velocity degrees of freedom $\{\underline{U}\}_{k}$ associated to a nonzero entry in the $j$-th row of matrix $C$, i.e., $C_{j k}>0$. Typically, such a block consists of all velocity degrees of freedom that are connected to a local element patch associated with the corresponding pressure degree of freedom; see Figure 1 (left) for a 2D situation. One Vanka-type smoothing iteration is a loop over all pressure degrees of freedom (vertices), where for each vertex the above local system is constructed and solved exactly. The linear system on the coarsest grid, i.e., the grid associated with the macro triangulation, is solved approximately by at most 10 iterations of the Vanka smoother.

5.1.2. Maxwell's equations. The magnetic field is computed from

$$
\begin{array}{r}
\left(\frac{\mathbf{B}^{n, \ell}-\mathbf{B}^{n-1}}{k}, \boldsymbol{\psi}\right)+\frac{1}{\bar{\mu}}\left(\frac{1}{\xi^{n-1}} \operatorname{curl}^{n, \ell}, \operatorname{curl} \psi\right) \\
-\left(\mathbf{U}^{n, \ell-1} \times \mathbf{B}^{n-1}, \operatorname{curl} \psi\right)=0
\end{array}
$$

where $\boldsymbol{\psi} \in \mathbf{X}_{h}$, and $\mathbf{X}_{h}$ is the space of lowest order edge elements. The simplified formulation (5.4) is obtained eliminating the Lagrange multiplier $R^{n, l}$ from (4.3). Such a simplification is justified if $\Omega$ is a convex polyhedron (see [11, Chapter 3.3.1]), which is the case in all our experiments. No stabilization was necessary in the formulation (5.4); cf. [10. An additional reason for not including the stabilization term in (5.4) is that it could have a negative effect on the discrete divergence 
free condition as it is inconsistent with the continuous formulation of Maxwell's equations; see also Remark 5.2 below. The conductivity is an elementwise constant function: $\left.\xi^{n-1}\right|_{K} \equiv \xi\left(\tilde{\rho}_{1}\right)$ if $\rho^{n-1}\left(\mathbf{x}_{K}\right)>\tilde{\rho}_{*}$ and $\left.\xi^{n-1}\right|_{K} \equiv \xi\left(\tilde{\rho}_{2}\right)$ otherwise, where $\mathbf{x}_{K}$ is the barycenter of the element $K \in \mathcal{T}_{h}$. The algebraic system associated with (5.4) is solved by a multigrid method with a patch smoother from [3]; see also 21. The construction of the edge element smoother is similar to the Vanka smoother described previously. One iteration of the edge element smoother consists of a loop over all vertices, where the local system associated with a given vertex contains all degrees for freedom (edges) that are connected to the vertex; see Figure 1 (right) for a $2 \mathrm{D}$ example.

Remark 5.2 (Divergence of magnetic field). The discrete approximation of Maxwell's equations (5.4) does not preserve the zero divergence of the magnetic field, i.e., $\operatorname{div} \mathbf{B}^{n, \ell} \neq 0$ in general. A good error indicator for the divergence free condition is the residual type quantity

$$
E_{\mathrm{div}} \equiv\left(\sum_{\chi \in S_{h} \equiv V_{h}}\left(\mathbf{B}^{n, \ell}, \nabla \chi\right)^{2}\right)^{1 / 2} .
$$

The worst case scenario for all our experiments was $E_{\mathrm{div}}=\mathcal{O}\left(10^{-3}\right)$.

Remark 5.3 (Lagrange vs. edge elements for Maxwell's equations). The theoretical analysis from Section 4 suggests that the $\mathbf{W}^{1,2}$-conforming finite element spaces might have better numerical properties than the $\mathbf{H}(\mathbf{c u r l})$-conforming edge elements. However, we found that the algebraic system resulting from a discretization of Maxwell's equations by quadratic finite elements was extremely difficult to solve iteratively for discontinuous conductivity. Following [14] we added a regularization term $\frac{1}{\mu}\left(\frac{1}{\xi^{n-1}} \operatorname{div} \mathbf{B}^{n, \ell}, \operatorname{div} \psi\right)$ to the Lagrange element formulations of (5.4), (4.3); however, the modification proved to be inefficient for a discontinuous conductivity. The same holds for the $\gamma$ stabilization term from (4.3), which in addition has a negative effect on the divergence-free constraint. On the other hand, the edge elements multigrid solver proved to be robust with respect to discontinuities in the conductivity; cf. 20. Moreover, in contrast to the above conjecture motivated from theory, we observe no significant differences in the convergence of the fixedpoint iterations for both types of finite element spaces; on average, five iterations were sufficient in order to satisfy the stopping criterion (5.6). Based on our experience and the above arguments, we conclude, that the edge elements seem to be a preferable choice for practical applications with discontinuous coefficients.

5.1.3. Density. The evolution of the density is computed from

$$
\left(\frac{\rho^{n, \ell}-\rho^{n-1}}{k}, \chi\right)_{h}+\left(\mathbf{U}^{n, \ell-1} \cdot \nabla \rho^{n, \ell}, \chi\right)+\left(\alpha_{\rho}\left(\mathbf{U}^{n, \ell-1}\right) \nabla \rho^{n, \ell}, \nabla \chi\right)=0 .
$$

The stabilization $\left(\alpha_{\rho}\left(\mathbf{U}^{n, \ell-1}\right) \nabla \rho^{n, \ell}, \nabla \chi\right)$ is crucial in order to obtain solutions free of spurious oscillations. An upwind scheme is obtained if we take the stabilization parameter as follows $\left.\alpha_{\rho}(\mathbf{U})\right|_{K}=\frac{h_{K}}{2} \| \mathbf{U}_{\mathbf{L}^{\infty}(K)}$. The above upwind scheme is a practical generalization of the original $\alpha$-term from (4.1) with $\alpha=1$. The upwind stabilization can be overdiffusive which is a drawback in applications where the evolution of interfaces is of interest. In the numerical experiments, (5.5) is solved by an algebraic flux correction scheme from [26], 25] where the parameter $\left.\alpha_{\rho}\right|_{K}$ 
is chosen adaptively on the algebraic level. The algebraic flux correction schemes retain the advantages of the upwind stabilization such as the mass conservation, and monotonicity while producing solutions with less numerical diffusion. Note, that we have dropped the source term ([div $\left.\left.\mathbf{U}^{n, \ell-1}\right] \rho^{n, \ell}, \chi\right)$ in the formulation (5.5)), as this had no observable influence on the properties of the scheme. Moreover, we found it numerically preferable to enforce mass conservation on the algebraic level within the framework of the algebraic flux correction scheme.

The discrete fluid interface for a given density approximation $\rho^{n}$ is defined as $\Gamma_{I}^{n}=\left\{\mathbf{x} \subset \Omega ; \rho^{n}(\mathbf{x})=\tilde{\rho}_{*}\right\}$. Further, we consider the discrete interfacial region

$$
\Omega_{I}^{n}=\bigcup\left\{K ; \exists\left(\mathbf{x}_{i} \in \mathcal{E}_{h}\right) \subset \bar{K} \text { such that } \tilde{\rho}_{2}<\rho^{n}\left(\mathbf{x}_{i}\right)<\tilde{\rho}_{1}\right\} .
$$

Note, that either $\rho^{n}=\tilde{\rho}_{1}$ or $\rho^{n}=\tilde{\rho}_{2}$ on $\Omega \backslash \Omega_{I}^{n}$. Ideally, the width of the interfacial region $\Omega_{I}^{n}$ should stay nearly uniform throughout the whole computation. However, the numerical diffusion and the variations of the velocity across $\Omega_{I}^{n}$ may ultimately lead to a distortion of the width of the interfacial region. To correct this unwanted "smearing" effect we propose the following postprocessing step combined with mesh adaptation at the end of every time step.

\section{Postprocessing step:}

- Loop over all vertices $\mathbf{x}_{i} \in \mathcal{E}_{h}$ :

(1) create a local patch $\mathcal{P}\left(\mathbf{x}_{i}\right)=\bigcup\left\{\bar{K} ; \mathbf{x}_{i} \subset \bar{K}\right\}$ of elements sharing the vertex $\mathbf{x}_{i}$;

(2) if $\mathcal{P}\left(\mathbf{x}_{i}\right) \cap \Gamma_{I}^{n}=\emptyset$ and if $\rho^{n}\left(\mathbf{x}_{i}\right)>\tilde{\rho}_{*}\left(<\tilde{\rho}_{*}\right)$, then set $\rho^{n}\left(\mathbf{x}_{i}\right)=$ $\max \left\{\tilde{\rho}_{1}, \tilde{\rho}_{2}\right\}\left(\min \left\{\tilde{\rho}_{1}, \tilde{\rho}_{2}\right\}\right)$ and mark for coarsening all $K^{*} \in \mathcal{P}\left(\mathbf{x}_{i}\right)$ for which $h_{K^{*}}<h_{\max }$

(3) if $\mathcal{P}\left(\mathbf{x}_{i}\right) \cap \Gamma_{I}^{n} \neq \emptyset$, mark for refinement all $K^{*} \in \mathcal{P}\left(\mathbf{x}_{l}\right)$ for which $\overline{K^{*}} \cap \Gamma_{I}^{n} \neq \emptyset$ and $h_{K^{*}}>h_{\min }$;

- refine/coarsen the mesh:

The prescribed parameters $h_{\min }, h_{\max }$ define the minimum and maximum mesh sizes, respectively. We choose $h_{\min }=1 / 32$ to ensure that the mesh is sufficiently fine in the interfacial region $\Omega_{I}^{n}$ in order to properly resolve the interface $\Gamma_{I}^{n}$, while the maximum mesh size is chosen $h_{\max }=1 / 8$ for better efficiency; see Figure 3 , The postprocessing step ensures that the width of the interfacial region remains approximately constant throughout the whole computations; see e.g., Figure 2 .

Remark 5.4 (Postprocessing and mass conservation). Without the postprocessing our method is mass conservative; however, the postprocessing inevitably leads to small changes in total mass. The relative mass error for the Rayleigh-Taylor instability experiment 5.2 was around 0.5 percent and around $10^{-4}$ percent for the two-fluid magnetohydrodynamics experiments 5.4. In general, there should be no need to perform the artificial postprocessing step if the computations were performed on sufficiently fine meshes. However, additional mesh refinement would render our 3D computations prohibitively expensive.

We use adaptive time stepping according to a local CFL number $C F L:=$ $k \max _{K \in \mathcal{T}_{h}}\left\|\mathbf{U}^{n}\right\|_{\mathbf{L}_{\infty}(K)} h_{K}^{-1}$. The time step size $k$ is chosen in such a way that $C F L<T O L_{C F L}$. The time steps in the numerical experiments were in the range $0.005 \leq k \leq 0.1$ for $T O L_{C F L}=0.8$. 

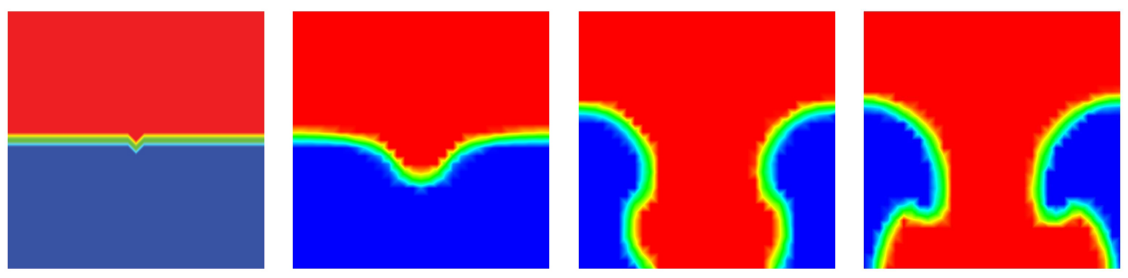

Figure 2. Density at $x=0.5$ at times $t=0,4,14,20$.
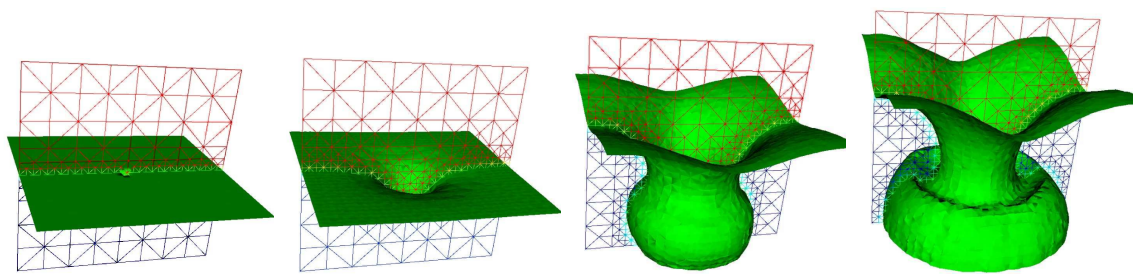

Figure 3. Fluid interface and mesh at $x=0.5$ at time $t=0,4,14,20$.

Instead of (4.4), we employ a more practical stopping criterion for the fixed point iterations in Algorithm A,

$$
\left\|\rho^{n, \ell-1}-\rho^{n, \ell}\right\|_{L^{\infty}}+\left\|\mathbf{B}^{n, \ell-1}-\mathbf{B}^{n, \ell}\right\|_{\mathbf{L}^{\infty}}+\left\|\mathbf{U}^{n, \ell-1}-\mathbf{U}^{n, \ell}\right\|_{\mathbf{L}^{\infty}} \leq \mathrm{TOL},
$$

with TOL $=10^{-8}$. Note, that on a discrete level (5.6) implies that (4.4) is satisfied with $\theta=C(h, k)$ TOL.

The complete adaptive algorithm for a fixed time level $n$ can be summarized as follows:

- Compute $\rho^{n}, \mathbf{U}^{n}, \mathbf{B}^{n}$ : employ Algorithm A with stopping criterion (5.6) and with (4.1), (4.2), (4.3) replaced by (5.5), (5.1), (5.4).

- Adapt time step: set $C F L=k \max _{K \in \mathcal{T}_{h}}\left\|\mathbf{U}^{n}\right\|_{\mathbf{L}_{\infty}(K)} h_{K}^{-1}$; if $C F L>$ $T O L_{C F L}$ set $k:=0.7 k$ else, if $C F L<0.01 T O L_{C F L}$ set $k:=1.5 k$.

- Perform the postprocessing step.

- Proceed to the next time level, set $n:=n+1$.

As is mentioned in Remark 5.3, we observe no significant dependence of the number of the fixed-point iterations on the ratio between the time step and the mesh size in the numerical experiments. However, it may not be expected that this property of the numerical algorithm remains true for smaller mesh sizes.

5.2. Rayleigh-Taylor instability. The following example is to test the performance of the two-fluid solver without the electromagnetic effects, i.e., we set $\mathbf{B} \equiv \mathbf{0}$. Rayleigh-Taylor instabilities are created when a heavy fluid is placed upon a lighter one and when the initial equilibrium state is perturbed. The computational domain is a unit cube. We take $\bar{\rho}_{1}=2235 \mathrm{~kg} / \mathrm{m}^{3}, \bar{\rho}_{2}=10^{-3} \bar{\rho}_{1}$. For the velocity we use the slip boundary condition (5.2), and zero initial condition. The evolution of the fluid density at $x=0.5$ is given in Figure 2, and the evolution of the fluid interface together with the evolution of the adaptive mesh can be seen in Figure 3 . 


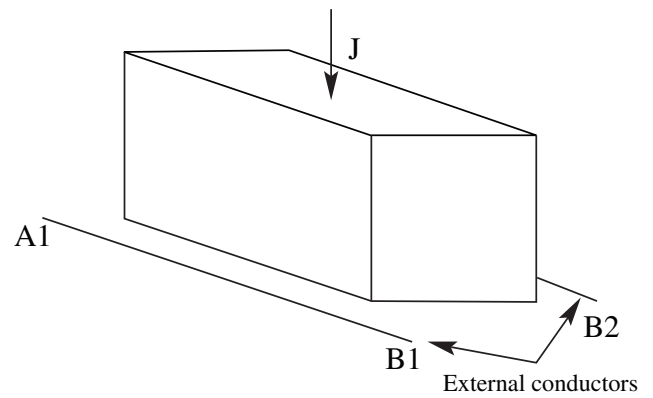

FiguRE 4. Experimental setup.

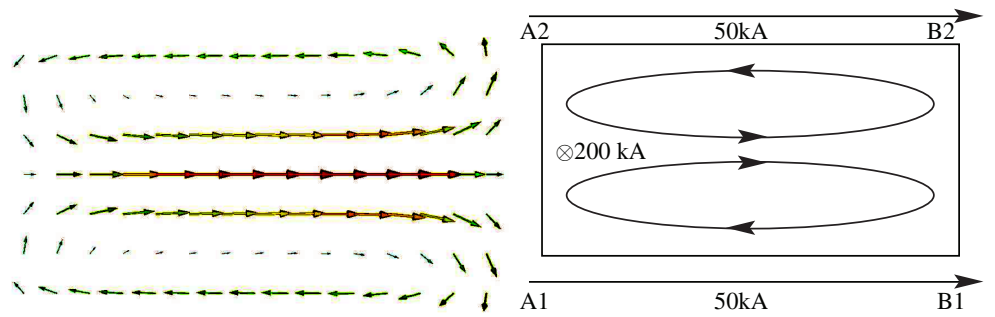

Figure 5 . Velocity at $z=0.25$ at time $t=5$ (left) and the predicted result (right).

5.3. Single fluid magnetohydrodynamics. In order to test the magnetohydrodynamic part of the code we perform single fluid experiments similar to the experiments from [10]. The computational domain $\Omega$ is a $2 \times 1 \times 1$ brick. There is a homogeneous electric current $\mathbf{J}=-(0,0,200) \mathrm{kA}$ flowing through the computational domain. In addition, two linear conductors carrying electric current are placed near the domain $\Omega$; see Figure 4 . The boundary condition for the magnetic field is obtained by computing the field generated by the electric current in the brick and in the external conductors using the Biot-Savart law. For the velocity we use a homogeneous Dirichlet boundary condition. We consider two configurations for the external conductors. The Figures 5 and 6 show the different configurations and their effect on the velocity field together with the expected results. Our transient computations qualitatively agree with the results from [10 which were obtained from a static model.

5.4. Two-fluid magnetohydrodynamics. In the next three experiments we study the evolution of an interface between two conducting fluids. The physical parameters for the two fluids are chosen close to those from [13, 32] in order to mimic the processes during aluminium electrolysis.

The domain $\Omega$ in the first experiment is a unit cube. We use the slip boundary condition (5.2) for the velocity field. For the magnetic field we use a nonlinear Neumann type boundary condition, we set

$$
\mathbf{n} \times\left(\frac{1}{\mu \xi(\rho)} \operatorname{curl} \mathbf{b}-\mathbf{u} \times \mathbf{b}\right)=(0,0,-0.5)
$$



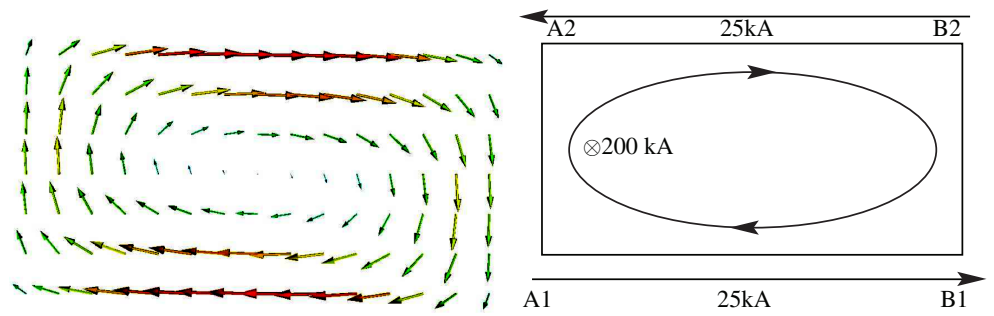

Figure 6 . Velocity at $z=0.25$ at time $t=5$ (left) and the predicted result (right).
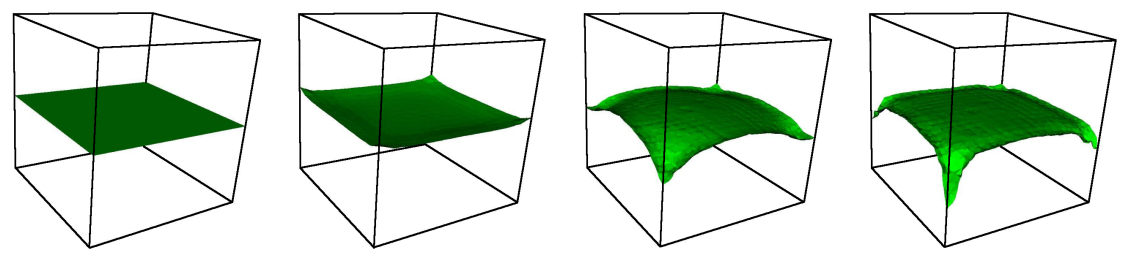

Figure 7. Fluid interface at time $t=0,2,7,10$.
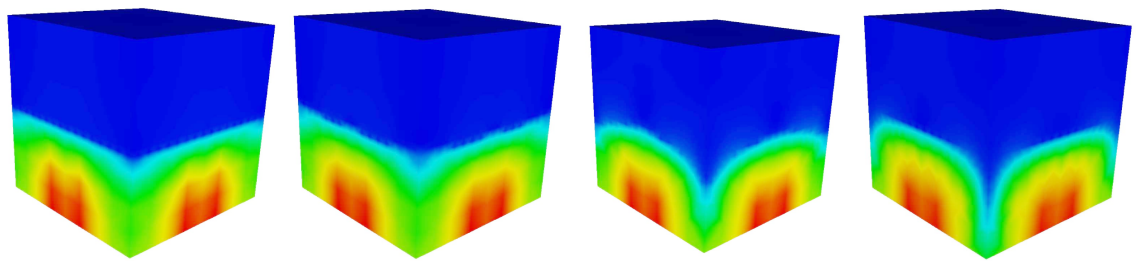

Figure 8. Magnitude of the magnetic field at time $t=0.1,2,7,10$.

on the horizontal walls, and

$$
\mathbf{n} \times\left(\frac{1}{\mu \xi(\rho)} \operatorname{curl} \mathbf{b}-\mathbf{u} \times \mathbf{b}\right)=\mathbf{0}
$$

on the top and bottom walls of the cube. Note, that the above choice of boundary condition represents a Dirichlet boundary condition for the normal component of the electric field $\mathbf{e}=\frac{1}{\mu \xi(\rho)} \mathbf{c u r l} \mathbf{b}-\mathbf{u} \times \mathbf{b}$. Both, the magnetic field and the velocity are zero at the beginning of the experiment. Initially, the lighter fluid (cryolite) with $\bar{\rho}_{1}=2090 \mathrm{~kg} / \mathrm{m}^{3}$ and $\bar{\xi}_{1}=10^{-3} \times \mu_{0}^{-1}$ is placed on top of the heavier fluid (liquid aluminium) with $\bar{\rho}_{2}=2235 \mathrm{~kg} / \mathrm{m}^{3}, \bar{\xi}_{2}=0.1 \times \mu_{0}^{-1}$. The described initial configuration of the two fluids represent an equilibrium if magnetic effects are neglected. The nonhomogeneous boundary condition on the vertical walls induces a strong magnetic field in the parts of the domain occupied by fluid with high conductivity, i.e., the liquid aluminium; see Figure 8 and Figure 10. In effect, a velocity field is created by the magnetic field, Figure 9. The flow is stronger in the liquid aluminium, and causes a deformation of the initially straight interface between the two fluids; see Figure 7 . 


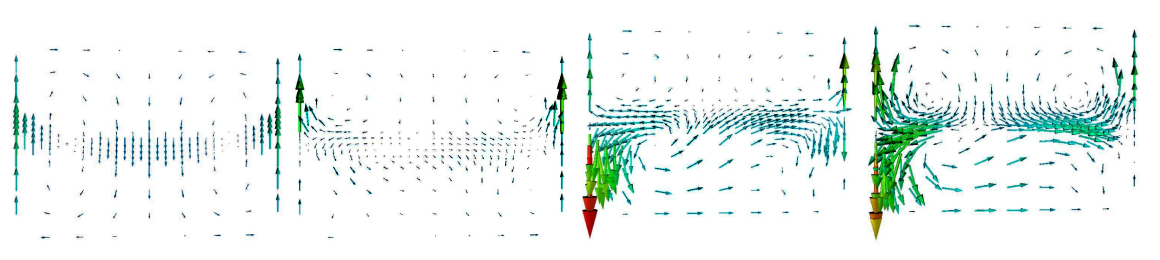

Figure 9. Velocity at $x+y=-1$ at time $t=0.1,2,7,10$.
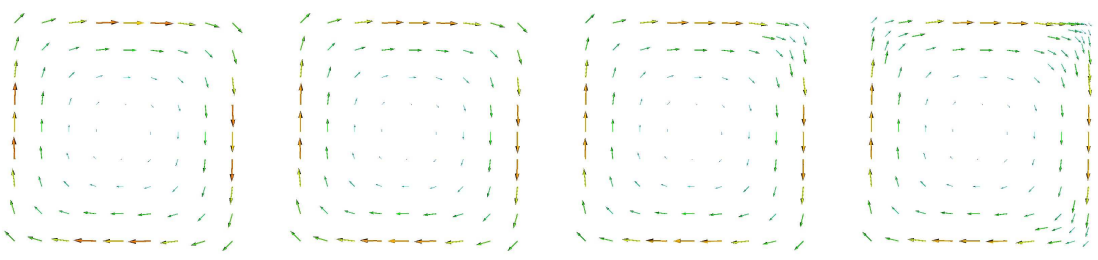

Figure 10. Magnetic field at $z=0.25$ at time $t=0.1,2,7,10$.
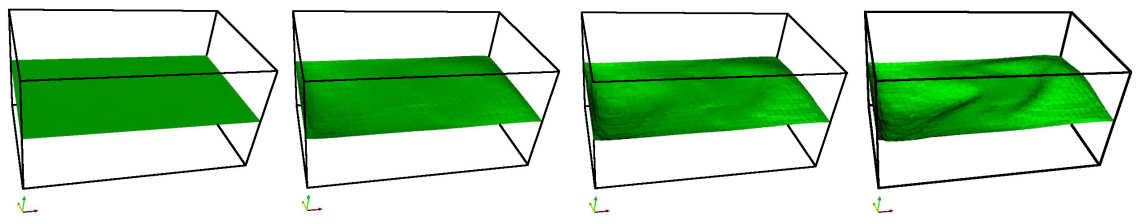

Figure 11. Fluid interface at time $t=0,2,3,4$.
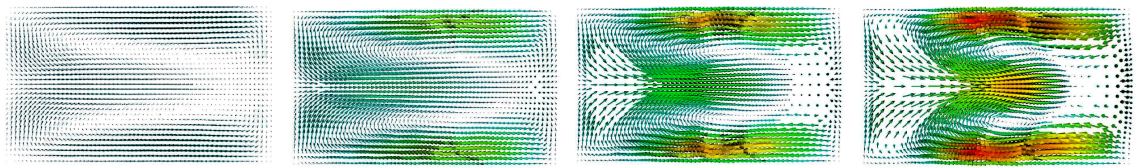

FiguRE 12 . Velocity at $z=0.5$ at time $t=1,2,3,4$.

The setup of the second experiment is based on the first single fluid experiment from Section [5.3. see Figure 4. Similarly, as in the previous two-fluid experiment, the domain $\Omega$ is occupied by two fluids with densities $\bar{\rho}_{1}=2090 \mathrm{~kg} / \mathrm{m}^{3}, \bar{\rho}_{2}=$ $2235 \mathrm{~kg} / \mathrm{m}^{3}$. The fluids are initially separated by a straight interface; see Figure 11] To demonstrate the ability of our method to deal with large conductivity jumps, we take $\bar{\xi}_{1}=10^{-5} \times \mu_{0}^{-1}, \bar{\xi}_{2}=0.1 \times \mu_{0}^{-1}$. We consider zero initial conditions for both the magnetic field and the velocity. A homogeneous Dirichlet boundary condition ("no slip") is prescribed for the velocity. The boundary condition for the magnetic field is obtained in the same fashion as in the single fluid case, i.e., from the Biot-Savart using the prescribed values of the electric current flowing through the domain and the external conductors; see Figure 5 (right). The evolution of the fluid interface is depicted in Figure 11. The evolutions of the velocity and the magnetic field at $z=0.5$ are displayed in Figures 12 and 13 , respectively. 

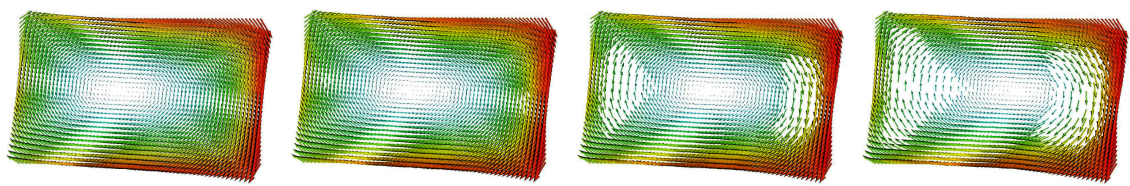

FIgURE 13. Magnetic field at $z=0.5$ at time $t=1,2,3,4$.
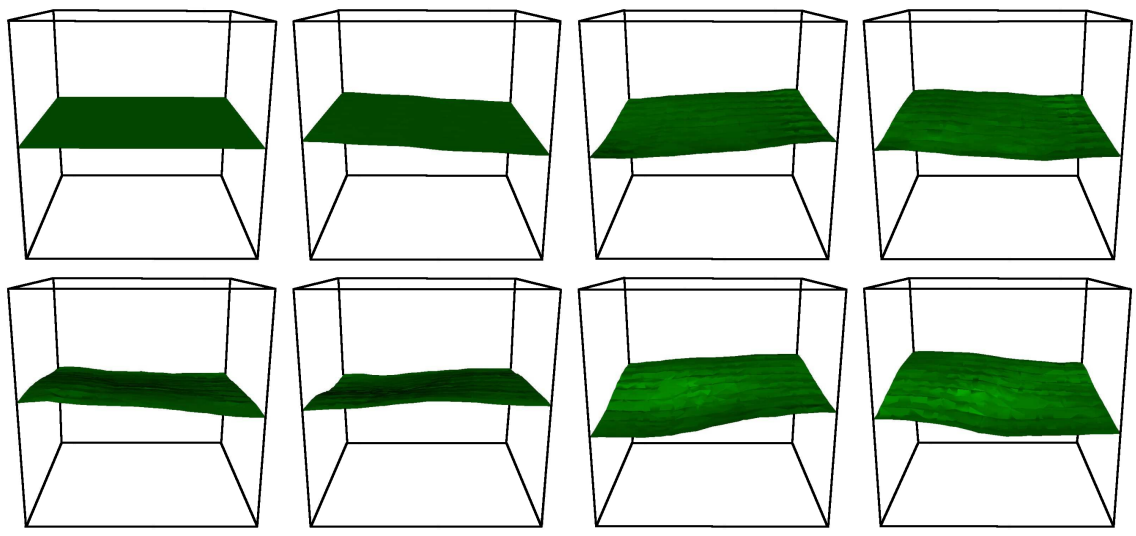

FiguRe 14. Interface at time $t=0,2,11,14,18,20,24,27$ for $b_{z}=0.1$.

In the final experiment we study the oscillations of the cryolite/aluminium interface, the so called "metal pad roll instabilities"; cf. [11, [32. The parameters for the computations are as follows: $\tilde{\rho}_{1}=0.935, \tilde{\rho}_{2}=\bar{\rho}_{\max }=1, \bar{\mu}=1, \bar{\xi}_{1}=10^{-4}$, $\bar{\xi}_{2}=1$. The domain $\Omega$ is a unit cube. The computations were performed on a uniform mesh with $h=1 / 16$ with fixed time step $k=0.1$. For the magnetic field we prescribe the following initial condition $\mathbf{B}^{0}(\mathbf{x})=(y-0.5,-(x-0.5), 0)$. The initial condition roughly corresponds to a magnetic field created by a uniform vertical electric current $\operatorname{curl} \mathbf{B}^{0}=(0,0,-2)$. For the velocity we prescribe the slip boundary condition and the initial condition is zero. To create an initial disturbance of the interface we set $\mathbf{g}=(0,-10 \sin (0.0556 \pi),-10 \cos (0.0556 \pi))$ for $0 \leq t \leq 1$, which is equivalent to "tilting" of the domain by an angle of $\approx 10^{\circ}$. The boundary condition for the magnetic field is $\mathbf{n} \times\left.\mathbf{B}\right|_{\partial \Omega}=\mathbf{n} \times\left.\mathbf{B}^{0}\right|_{\partial \Omega}$ for $0 \leq t \leq 1$. For $t>1$ the gravity is set straight, i.e., $\mathbf{g}=(0,0,-10)$ and a uniform magnetic field $\mathbf{B}_{z}=\left(0,0, b_{z}\right)$ is superimposed to the magnetic field created by the electric current, i.e., the boundary condition is changed to $\mathbf{n} \times\left.\mathbf{B}\right|_{\partial \Omega}=\mathbf{n} \times\left.\left(\mathbf{B}^{0}+\mathbf{B}_{z}\right)\right|_{\partial \Omega}$ for $1<t \leq 25$. We found that the oscillations were stable for $b_{z}=0.1$ and became unstable for the increased background magnetic field $b_{z}=0.2$. We display the position of the interface at various times for $b_{z}=0.1$ in Figure 14. Similarly, as in [11, we observe a formation of loops in the perturbed current $\operatorname{curl} \mathbf{B}-\operatorname{curl}^{0} \mathbf{B}^{0}$, which is predicted by the theoretical explanations of the rolling phenomenon; see Figure 15 . 


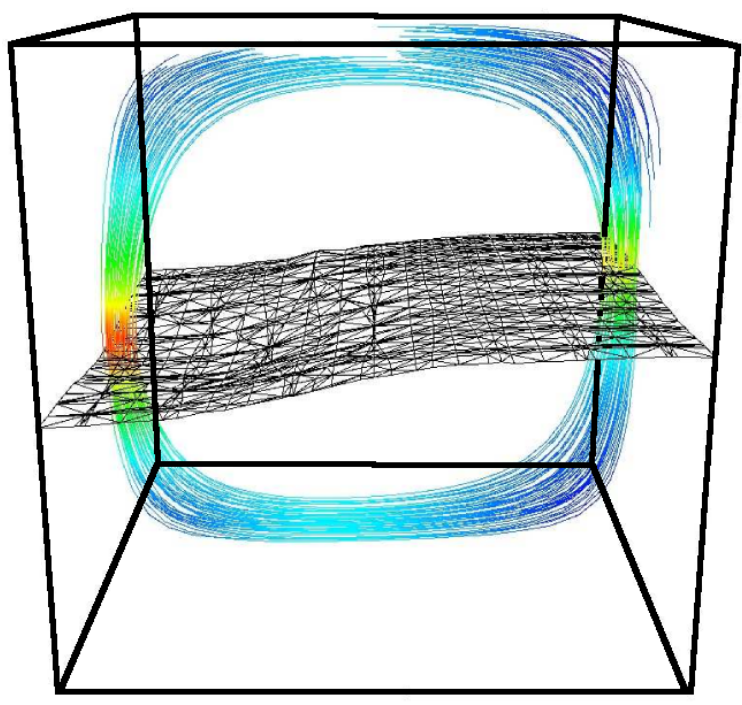

Figure 15. Streamlines of the perturbed current $\operatorname{curl} \mathbf{B}-\operatorname{curl}^{0} \mathbf{B}^{0}$ at time $t=22.3$. The current flows from right to left.

\section{REFERENCES}

[1] C. Amrouche, C. Bernardi, M. Dauge, V. Girault, Vector potentials in three-dimensional nonsmooth domains, Math. Meth. Appl. Sci. 21, pp. 823-864 (1998). MR.1626990|(99e:35037)

[2] F. Armero, J.C. Simo, Long-term dissipativity of time-stepping algorithms for an abstract evolution equation with applications to the incompressible MHD and Navier-Stokes equations, Comp. Meth. Appl. Mech. Engrg. 131, pp. 41-90 (1996). MR.1393572 (97g:76050)

[3] D. N. Arnold, R. S. Falk, R. Winther: Multigrid in $\mathrm{H}$ (div) and H(curl). Numer. Math., Vol. 85, pp. 197-217, 2000. MR1754719 (2001d:65161)

[4] S.C. Brenner, L.R. Scott, The mathematical theory of finite element methods, Springer (1994). MR:1278258 (95f:65001)

[5] Z. Chen, Q. Du, J. Zou, Finite element methods with matching and nonmatching meshes for Maxwell equations with discontinuous coefficients, SIAM J. Numer. Anal. 37, pp. 1542-1570 (2000). MR:1759906 (2001h:78044)

[6] J.F. Ciavaldini, Analyse numerique d'un probleme de Stefan a deux phases par une methode d'elements finis, SIAM J. Numer. Anal. 12, pp. 464-487 (1975). MR0391741 (52:12561)

[7] M. Costabel, M. Dauge, Weighted regularization of Maxwell equations in polyhedral domains, Numer. Math. 93, pp. 239-277 (2002). MR.1941397 (2004a:78039)

[8] M. Crouzeix, V. Thomée, The stability in $L_{p}$ and $W_{p}^{1}$ of the $L_{2}$-Projection onto finite element function spaces, Math. Comp. 48, pp. 521-532 (1987). MR878688 (88f:41016)

[9] R. DiPerna, P.L.-Lions, Ordinary differential equations, transport theory and Sobolev spaces, Invent. Math. 98, pp. 511-547 (1989). MR1022305 (90j:34004)

[10] J.-F. Gerbeau, A stabilized finite element method for the incompressible magnetohydrodynamic equations, Num. Math. 87, pp. 83-111 (2000). MR1800155 (2001j:76071)

[11] J.-F. Gerbeau, C. Le Bris, T. Lelievre, Mathematical methods for the magnetohydrodynamics of liquid metals, Oxford Science Publications (2006). MR2289481(2008i:76205)

[12] V. Girault, R.H. Nochetto, R. Scott, Maximum-norm stability of the finite element Stokes projection, J. Math. Pures Appl. 84, pp. 279-330 (2005). MR2121575 (2006j:76087)

[13] J.-F. Gerbeau, T. Lelièvre, and C. Le Bris. Simulations of MHD flows with moving interfaces, J. Comput. Phys. 184, pp. 163-191 (2003). MR1961974 (2003k:76158)

[14] D.T. Graves, D. Trebotich, G.H. Miller, P. Colella. An efficient solver for the equations of resistive MHD with spatially-varying resistivity, J. Comput. Phys. 227, pp. 4797-4804 (2008). $\operatorname{MR} 2414835$ 
[15] V. Girault, P.-A. Raviart, Finite element methods for Navier-Stokes equations, Springer (1986). MR851383 (88b:65129)

[16] M.D. Gunzburger, A.J. Meir, J.S. Peterson, On the existence and uniqueness and finite element approximation of solutions of the equations of stationary incompressible magnetohydrodynamics, Math. Comp. 56, pp. 523-563 (1991). MR1066834(91m:76127)

[17] U. Hasler, A. Schneebeli, D. Schötzau, Mixed finite element approximation of incompressible MHD problems based on weighted regularization, Appl. Num. Math. 51, pp. 19-45 (2004). MR2083323 (2005d:65209)

[18] J.G. Heywood, R. Rannacher, Finite element solution of the nonstationary Navier-Stokes problem. I. Regularity of solutions and second-order error estimates for spatial discretization, SIAM J. Numer. Anal. 19, pp. 275-311 (1982). MR650052 (83d:65260)

[19] W. Hackbusch. Multigrid methods and applications, volume 4 of Springer Series in Computational Mathematics. Springer-Verlag, Berlin (1985). MR814495(87e:65082)

[20] R. Hiptmair, Finite elements in computational electromagnetism, Acta Numerica 11, pp. 237-339 (2002). MR2009375 (2004k:78028)

[21] R. Hiptmair Multigrid method for Maxwell's equations, SIAM J. Numer. Anal. 36, pp. 204225 (1998). MR.1654571 (99j:65229)

[22] V. John. Higher order finite element methods and multigrid solvers in a benchmark problem for the 3d Navier-Stokes equations. Int. J. Numer. Meth. Fluids 40, pp. 775-798 (2002). MR1928952 (2003h:76092)

[23] F. Kikuchi, On a discrete compactness property for the Nédélec finite elements, J. Fac. Sci. Univ. of Tokyo Sec. IA 36, pp. 479-490 (1989). MR1039483 (91h:65173)

[24] S. Korotov, M. Krizek, Acute type refinements of tetrahedral partitions of polyhedral domains, SIAM J. Numer. Anal. 39, pp. 724-733 (2001). MR.1860255 (2002g:65144)

[25] Dmitri Kuzmin, On the design of general-purpose flux limiters for finite element schemes. I. Scalar convection. J. Comput. Phys., 219:513-531, 2006. MR2274948 (2007g:76134)

[26] Dmitri Kuzmin and Matthias Möller, Algebraic flux correction. I. Scalar conservation laws. In Flux-corrected transport, Sci. Comput., pages 155-206. Springer, Berlin, 2005. MR2129255

[27] J.L. Lions, Quelques methodes de resolution des problemes aux limites non lineaires, Dunod, Gauthier-Villars, Paris (1969). MR0259693 (41:4326)

[28] P.L. Lions, Mathematical topics in fluid mechanics, Volume I: Incompressible Models, Oxford University Press, Oxford (1996). MR.1422251 (98b:76001)

[29] J.L. Lions, E. Magenes, Nonhonmogeneous boundary value problems and applications, Volume I, Springer-Verlag, New York (1972).

[30] C. Liu, N.J. Walkington, Convergence of numerical approximations of the incompressible Navier-Stokes equations with variable density and viscosity, SIAM J. Numer. Anal. 45, pp. 1287-1304 (2007). MR2318813 (2008f:65177)

[31] P. Monk, Finite element methods for Maxwell's equations, Oxford University Press, New York (2003). MR2059447 (2005d:65003)

[32] D. Munger and A. Vincent. A level set approach to simulate magnetohydrodynamic instabilities in aluminium reduction cells, J. Comput. Phys. 217, pp. 295-311 (2006). MR.2260603 (2007f:76151)

[33] A. Prohl, Convergent finite element discretizations of the nonstationary incompressible magnetohydrodynamics system, Math. Mod. Numer. Analysis 42, pp. 1065-1087 (2008). MR2473320

[34] A. Schmidt and K. G. Siebert, ALBERT-software for scientific computations and applications, Acta Math. Univ. Comenian. (N.S.) 70, pp. 105-122 (2000). MR.1865363

[35] R.E. Showalter, Monotone operators in Banach space and nonlinear partial differential equations, AMS (1997). MR1422252 (98c:47076)

[36] D. Schötzau, Mixed finite element methods for stationary incompressible magnetohydrodynamics, Num. Math. 96, pp. 771-800 (2004). MR2036365 (2005b:76088)

[37] J. Simon, Sobolev, Besov and Nikolskii fractional spaces: Imbeddings and comparisons for vector valued spaces on an interval, Ann. Mat. Pura Appl. 157, pp. 117-148 (1990). MR:1108473 (92e:46075)

[38] N.J. Walkington, Convergence of the discontinuous Galerkin method for discontinuous solutions, SIAM J. Numer. Anal. 42, pp. 1801-1817 (2004). MR2139223 (2006b:65147)

[39] J. Zhao, Analysis of finite element approximation for time-dependent Maxwell problems, Math. Comp. 73, pp. 1089-1105 (2003). MR2047079 (2005b:65112) 
Department of Mathematics and the Maxwell Institute for Mathematical Sciences, Heriot-Watt University, EH14 4AS Edinburgh, United Kingdom

E-mail address: 1.banas@hw.ac.uk

Mathematisches Institut der Universität Tübingen, Auf Der Morgenstelle 10, D-72076 Tübingen, Germany

E-mail address: prohl@na.uni-tuebingen.de 